\title{
The branching bifurcation of Adaptive Dynamics
}

\author{
Fabio Della Rossa ${ }^{\dagger}$, Fabio Dercole ${ }^{\dagger *}$, Pietro Landi ${ }^{\ddagger}$ \\ ${ }^{\dagger}$ Department of Electronics, Information, and Bioengineering, Politecnico di Milano, Italy \\ ${ }_{\ddagger}^{\ddagger}$ Department of Mathematical Sciences, Stellenbosch University, South Africa and \\ Evolution and Ecology, International Institute for Applied Systems Analysis, Austria \\ *corresponding author, fabio.dercole@polimi.it
}

Received (to be inserted by publisher)

\begin{abstract}
We unfold the bifurcation involving the loss of evolutionary stability of an equilibrium of the canonical equation of Adaptive Dynamics (AD). The equation deterministically describes the expected long-term evolution of inheritable traits - phenotypes or strategies - of coevolving populations, in the limit of rare and small mutations. In the vicinity of a stable equilibrium of the $\mathrm{AD}$ canonical equation, a mutant type can invade and coexist with the present-resident- types, whereas the fittest always win far from equilibrium. After coexistence, residents and mutants effectively diversify, according to the enlarged canonical equation, only if natural selection favors outer rather than intermediate traits - the equilibrium being evolutionarily unstable, rather than stable. Though the conditions for evolutionary branching - the joint effect of resident-mutant coexistence and evolutionary instability — have been known for long, the unfolding of the bifurcation remained a missing tile of $\mathrm{AD}$, the reason being related to the nonsmoothness of the mutant invasion fitness after branching. In this paper, we develop a methodology that allows the approximation of the invasion fitness after branching in terms of the expansion of the (smooth) fitness before branching. We then derive a canonical model for the branching bifurcation and perform its unfolding around the loss of evolutionary stability. We cast our analysis in the simplest (but classical) setting of asexual, unstructured populations living in an isolated, homogeneous, and constant abiotic environment; individual traits are one-dimensional; intra- as well as inter-specific ecological interactions are described in the vicinity of a stationary regime.
\end{abstract}

Keywords: adaptive dynamics; bifurcation; evolutionary branching; evolutionary stability; invasion fitness; singular strategy. 


\section{Introduction}

2 Since its founding publications [Metz et al., 1996, Geritz et al., 1997, 1998], Adaptive Dynamics (AD) has been widely used for modeling the long-term evolutionary dynamics of genetically transmitted phenotypic

4 traits (see Dercole \& Rinaldi [2008] and the refs. therein), with special emphasis on the emergence of diversity through evolutionary branching. Evolutionary branching takes place when a resident and a similar mutant type coexist in the same environment and natural selection is disruptive, i.e., it favors outer rather than intermediate phenotypes. Series of subsequent mutations hence lead to the diversification of the two traits. Analogous phenomena can be observed in socio-economic contexts [Dercole et al., 2008, 2010b, Landi \& Dercole, 2014], where behavioral strategies, innovations, and competition play the role of phenotypic 10 traits, mutations, and natural selection [Ziman, 2000].

In the limit of extremely rare mutations of infinitesimal effect, evolution can be approximated by a continuous dynamics in terms of an ODE model, called the canonical equation of AD [Dieckmann \& Law, 1996, Champagnat et al., 2006]. The AD canonical equation describes the expected long-term evolution as 14 an ascent of the traits on an adaptive fitness landscape [Levins, 1968, Metz et al., 1992, Gavrilets, 2004]. All kinds of evolutionary attractors can be displayed, from stationary - called singular strategies in the AD 16 jargon - to periodic [Dieckmann et al., 1995, Dercole et al., 2003] and chaotic [Dercole et al., 2010a, Dercole \& Rinaldi, 2010]; and attractor multiplicity (ecological and/or evolutionary) questions the replicability of 18 evolutionary history [Dercole et al., 2002, 2006]. When mutational steps are finite and stochastically drawn, evolution proceeds as a random walk in the trait space of coevolving populations, again showing the full

20 plethora of nonlinear behaviors (see e.g. [Dieckmann, 1997, Doebeli \& Ruxton, 1997, Doebeli \& Dieckmann, 2000]).

22 Evolutionary branching can be formally described in terms of the stability properties of the singular strategies, seen as the evolutionary equilibria of the AD canonical equation. Specifically, resident-mutant 24 coexistence can only occur in the vicinity of a singular strategy [Geritz, 2005, Meszéna et al., 2005, Dercole \& Rinaldi, 2008], that must hence be a stable equilibrium - convergence stability in the AD jargon [Eshel, 1983,

${ }_{26}$ Taylor, 1989, Christiansen, 1991, Eshel et al., 1997] — to be reached by the evolutionary dynamics (unstable equilibria - convergence unstable singular strategies - are not considered). And it must be unstable for

28 the higher-dimensional canonical equation used after resident-mutant coexistence - evolutionary instability [Maynard Smith \& Price, 1973] — to produce phenotypic divergence. Whereas branching cannot occur if

30 coexistence is not possible close to the evolutionary equilibrium or if the equilibrium is evolutionarily stable - the equilibrium then represents a terminal point of the evolutionary dynamics [Dercole \& Rinaldi, 32 2008].

The transition from terminal to branching point (or vice-versa) along with changes in the relevant demographic, environmental, or control parameters, can therefore be interpreted as a bifurcation of the $\mathrm{AD}$ canonical equation. The unfolding of the bifurcation is however a missing tile of AD theory. The reason why it has been left behind is related to difficulties in developing a suitable normal form for the bifurcation. In fact, the fitness landscape after branching is nonsmooth at the branching point and this prevents the Taylor expansion approach typical of normal form analysis.

In this paper we develop a methodology that allows the approximation of the dimorphic fitness landscape - the invasion fitness of a mutant in the presence of two resident types at incipient branching - in terms of the expansion of the monomorphic fitness - the invasion fitness before branching - up to any order 42 locally to the branching point. We cast our analysis in the simplest (but classical) setting of unstructured populations (no distinction in age, state, location, etc., of individuals) varying in continuous time in an iso44 lated, homogeneous, and constant abiotic environment; individual traits are quantified by one-dimensional strategies; intra- as well as inter-specific ecological interactions are described in the vicinity of a stationary 46 regime. We exploit an expansion in the radial direction in the plane of the two coexisting strategies and show that the fitness landscape is smooth on each given ray, thus obtaining an approximation that is 48 parametric in the ray angle.

By means of a third-order approximation, we unfold the branching bifurcation involving the change in 50 evolutionary stability of the singular strategy. In particular, the third derivative of the monomorphic fitness with respect to the mutant strategy is the critical coefficient ruling branching at the bifurcation. The other 
transition from terminal to branching point, the one involving the possibility of resident-mutant coexistence 2 near the singularity, is more intricate and is left for future research. This, as well as bifurcations of higher codimension (more degeneracies occurring together), can in principle be dealt with the same methodology.

Interestingly, our approximation coincides up to second-order to the one obtained by Geritz et al. [1997, 1998] by assuming a smooth dimorphic fitness (see the Appendix 1 in [Geritz et al., 1998] in particular). 6 Thus, the branching conditions derived by Geritz et al. [1997, 1998], in terms of the second derivatives of the monomorphic fitness at the singular strategy, are confirmed. The third-order terms in the approximation however differ from those one would obtain under the smoothness assumption. Worth to remark is that our third-order terms are given in terms of the monomorphic fitness derivatives (in contrast to what preliminarily found by Durinx [2008], in the special case of Lotka-Volterra models), so the evolutionary dynamics locally to a branching point are determined by quantities that can be evaluated without waiting

12 for the mutation that triggers the branching - an important feature for the empirical test of evolutionary predictions.

The paper is organized as follows. In the next section we introduce the basic notation and the methodology used for approximating the dimorphic invasion fitness. For simplicity, we consider a single species generic model (as done in [Geritz et al., 1997, 1998]) and we focus on the transition from the monomorphic to the dimorphic situation. In Appendix C, we consider higher polymorphisms and/or inter-specific ecological and/or coevolutionary interactions. The results are fully analogous, but more involved to be derived. In Sects. 3 and 4 we present the normal form and the unfolding of the branching bifurcation, respectively.

${ }_{20}$ Sect. 5 is dedicated to two examples, where the developed theory is applied to two AD models taken from the literature. Finally, in Sect.6, we discuss possible extensions for future work. In particular, similar results are expected to hold for the case of structured populations characterized by multi-dimensional strategies. All analytical computations have been handled symbolically and a fully-commented Mathematica script is provided as online Supplementary Material.

\section{Methods}

\subsection{Notation, assumptions, and preliminaries}

We consider two similar competing populations, with abundances measured by densities $n_{1}(t)$ and $n_{2}(t)$ populations 1 and 2 (the case in which other conspecific populations and/or other species are present so is treated in Appendix C). Before branching, we refer to populations 1 and 2 as resident and mutant, respectively, whereas they are both residents after branching.

Populations 1 and 2 being conspecific, their per-capita growth rates $\dot{n}_{1} / n_{1}$ and $\dot{n}_{2} / n_{2}$ can be expressed through the same fitness generating function (or $g$-function [Vincent \& Brown, 2005]) $g\left(n_{1}, n_{2}, x_{1}, x_{2}, x^{\prime}\right)$ the per-capita growth rate of a virtual population with strategy $x^{\prime}$ and infinitesimally small density in the environment set by strategies $x_{1}$ and $x_{2}$ with densities $n_{1}$ and $n_{2}$. Then, $\dot{n}_{1} / n_{1}$ and $\dot{n}_{2} / n_{2}$ are given by the $g$-function evaluated for $x^{\prime}=x_{1}$ and $x^{\prime}=x_{2}$, respectively:

$$
\begin{aligned}
& \dot{n}_{1}=n_{1} g\left(n_{1}, n_{2}, x_{1}, x_{2}, x_{1}\right), \\
& \dot{n}_{2}=n_{2} g\left(n_{1}, n_{2}, x_{1}, x_{2}, x_{2}\right),
\end{aligned}
$$

the resident-mutant model of AD [Dercole \& Rinaldi, 2008].

To define reasonable population dynamics, function $g$ enjoys the four basic properties P1-P4 summarized below. The first three are rather obvious, whereas the fourth one is more involved and has been 40 recently introduced [Dercole, 2014]. We assume $g$ to be smooth and we use lists of integer superscripts to indicate the arguments w.r.t. which we take derivatives and the order of differentiation, e.g.

$$
\begin{aligned}
& g^{(1,0,0,0,0)}\left(n_{1}, n_{2}, x_{1}, x_{2}, x^{\prime}\right):=\frac{\partial}{\partial n_{1}} g\left(n_{1}, n_{2}, x_{1}, x_{2}, x^{\prime}\right), \\
& g^{(1,1,0,0,0)}\left(n_{1}, n_{2}, x_{1}, x_{2}, x^{\prime}\right):=\frac{\partial^{2}}{\partial n_{1} n_{2}} g\left(n_{1}, n_{2}, x_{1}, x_{2}, x^{\prime}\right), \\
& g^{(2,0,0,0,0)}\left(n_{1}, n_{2}, x_{1}, x_{2}, x^{\prime}\right):=\frac{\partial^{2}}{\partial n_{1}^{2}} g\left(n_{1}, n_{2}, x_{1}, x_{2}, x^{\prime}\right) .
\end{aligned}
$$

${ }_{42} \mathrm{P} 1: g\left(n_{1}, 0, x_{1}, x_{2}, x^{\prime}\right)=g_{1}\left(n_{1}, x_{1}, x^{\prime}\right)$, 
for a suitable function $g_{1}$, i.e., the per-capita growth rate of a strategy $x^{\prime}$ is not affected by the strategy $2 \quad x_{2}$ of an absent population.

P2: $g\left(n_{1}, n_{2}, x, x, x^{\prime}\right)=g\left(\alpha\left(n_{1}+n_{2}\right),(1-\alpha)\left(n_{1}+n_{2}\right), x, x, x^{\prime}\right)$,

for any $0 \leq \alpha \leq 1$, i.e., any partition of the total density $\left(n_{1}+n_{2}\right)$ into two populations with same strategy $x$ must result in the same per-capita growth rate for strategy $x^{\prime}$.

6 P3: $g\left(n_{1}, n_{2}, x_{1}, x_{2}, x^{\prime}\right)=g\left(n_{2}, n_{1}, x_{2}, x_{1}, x^{\prime}\right)$,

i.e., the order in which populations 1 and 2 are considered does not matter.

8 $\mathrm{P} 4: \quad g^{\left(0,0, d_{1}, 0,0\right)}\left(n_{1}, n_{2}, x, x, x^{\prime}\right)=\sum_{i_{1}=1}^{d_{1}} \phi_{d_{1}, i_{1}}\left(n_{1}+n_{2}, x, x^{\prime}\right) n_{1}^{i_{1}}$,

$g^{\left(0,0, d_{1}, d_{2}, 0\right)}\left(n_{1}, n_{2}, x, x, x^{\prime}\right)=\sum_{i_{1}=1}^{d_{1}} \sum_{i_{2}=1}^{d_{2}} \phi_{d_{1}, d_{2}, i_{1}, i_{2}}\left(n_{1}+n_{2}, x, x^{\prime}\right) n_{1}^{i_{1}} n_{2}^{i_{2}}$,

for suitable functions $\phi_{d_{1}, i_{1}}$ and $\phi_{d_{1}, d_{2}, i_{1}, i_{2}}, d_{1}, d_{2} \geq 1$. This property follows from a generalized principle of mass-action, i.e., the assumption that $g$ describes the pairwise interactions of a virtual individual with strategy $x^{\prime}$ with $x_{1,2}$-individuals involved, in turn, in other pairwise interactions [Dercole, 2014].

Properties $\mathrm{P} 1-\mathrm{P} 4$ can be combined to produce further relations among $g$-derivatives that will be taken into account in our expansions in Sect.2.3 (in particular in the Supplementary Material). For example:

141,2a: $g^{\left(l_{1}, l_{2}, 0,0,0\right)}\left(n_{1}, n_{2}, x, x, x^{\prime}\right)=g_{1}^{\left(l_{1}+l_{2}, 0,0\right)}\left(n_{1}+n_{2}, x, x^{\prime}\right)$,

i.e., $n_{1}$ - and $n_{2}$-perturbations simply perturb the total density $\left(n_{1}+n_{2}\right)$ if the two populations have 16 the same strategy $x$.

P1,2b: $\sum_{i=0}^{d}\left(\begin{array}{l}d \\ i\end{array}\right) g^{\left(l_{1}, l_{2}, i, d-i, 0\right)}\left(n_{1}, n_{2}, x, x, x^{\prime}\right)=g_{1}^{\left(l_{1}+l_{2}, d, 0\right)}\left(n_{1}+n_{2}, x, x^{\prime}\right)$,

$18 \quad d \geq 1$, obtained by $x$-differentiating P1-2a.

$\mathrm{P} 1,3: g\left(0, n_{2}, x_{1}, x_{2}, x^{\prime}\right)=g_{1}\left(n_{2}, x_{2}, x^{\prime}\right)$.

${ }_{20} \mathrm{P} 1,4: g_{1}^{(0, d, 0)}\left(n, x, x^{\prime}\right)=\sum_{i=1}^{d} \phi_{d, i}\left(n, x, x^{\prime}\right) n^{i}$.

$\mathrm{P} 1,2,4: \sum_{i=i_{1}}^{d-i_{2}}\left(\begin{array}{c}d \\ i\end{array}\right) \phi_{i, d-i, i_{1}, i_{2}}\left(n, x, x^{\prime}\right)=\left(\begin{array}{c}i_{1}+i_{2} \\ i_{1}\end{array}\right) \phi_{d, i_{1}+i_{2}}\left(n, x, x^{\prime}\right)$,

22 for each $i_{1}, i_{2} \geq 1$ with $i_{1}+i_{2} \leq d \geq 2$, obtained by substituting $\mathrm{P} 4$ and P1,4 into P1,2b (with $\left.l_{1}=l_{2}=0\right)$ and by balancing same $\left(n_{1}, n_{2}\right)$-monomials at the left- and right-hand sides. In particular,

$$
\begin{aligned}
& d=2 \quad i_{1}=1 \quad i_{2}=1 \quad \text { gives } \quad 2 \phi_{1,1,1,1}=2 \phi_{2,2}, \\
& d=3 \quad i_{1}=1 \quad i_{2}=1 \quad \text { gives } \quad 3 \phi_{1,2,1,1}+3 \phi_{2,1,1,1}=2 \phi_{3,2}, \\
& d=3 \quad i_{1}=1 \quad i_{2}=2 \quad \text { gives } \quad 3 \phi_{1,2,1,2}=3 \phi_{3,3}, \\
& d=3 \quad i_{1}=2 \quad i_{2}=1 \quad \text { gives } \quad 3 \phi_{2,1,2,1}=3 \phi_{3,3},
\end{aligned}
$$

thus linking the functions $\phi$ 's with two sum indexes to those characterized by a single sum index.

P63,4a: $g^{\left(0,0,0, d_{2}, 0\right)}\left(n_{1}, n_{2}, x, x, x^{\prime}\right)=\sum_{i_{2}=1}^{d_{2}} \phi_{d_{2}, i_{2}}\left(n_{1}+n_{2}, x, x^{\prime}\right) n_{2}^{i_{2}}$.
P3,4b: $\phi_{d_{1}, d_{2}, i_{1}, i_{2}}=\phi_{d_{2}, d_{1}, i_{2}, i_{1}}$.

${ }_{2} \mathrm{P} 1-4: \quad \phi_{1,1,1,1}=\phi_{2,2}, \quad \phi_{2,1,1,1}=\phi_{1,2,1,1}=\frac{1}{3} \phi_{3,2}, \quad \phi_{2,1,2,1}=\phi_{1,2,1,2}=\phi_{3,3}$, obtained by exploiting P3,4b in the examples of P1,2,4.

30 Moreover, further derivatives w.r.t. to the virtual strategy $x^{\prime}$ can be added to all properties.

As anticipated in the Introduction, we consider the (simplest, but most typical) case of stationary 32 coexistence. In particular, we assume that for all values of the strategy $x_{1}$ that we consider, the resident population 1 can persist alone at a strictly positive and (hyperbolically) stable equilibrium of Eq. (1a) with 
$n_{2}=0$. We denote the equilibrium density with function $\bar{n}\left(x_{1}\right)$, implicitly defined by

$$
g\left(\bar{n}\left(x_{1}\right), 0, x_{1}, x_{2}, x_{1}\right) \stackrel{\text { P1 }}{=} g_{1}\left(\bar{n}\left(x_{1}\right), x_{1}, x_{1}\right)=0 .
$$

2 Note that the hyperbolic stability of the resident equilibrium (i.e., $\left.g_{1}^{(1,0,0)}\left(\bar{n}\left(x_{1}\right), x_{1}, x_{1}\right)<0\right)$ and the similarity between the resident and mutant populations $\left(x_{1} \simeq x_{2}\right)$, guarantee that population 2 is also 4 able to persist alone at the strictly positive (and hyperbolically stable) equilibrium $\bar{n}\left(x_{2}\right)$ of Eq. (1b) with $n_{1}=0$ (function $\bar{n}\left(x_{1}\right)$ is uniquely defined, locally to $x_{1}$, by the implicit function theorem). In other words, ${ }_{6}$ the resident-mutant model (1) admits the two monomorphic equilibria $\left(\bar{n}\left(x_{1}\right), 0\right)$ and $\left(0, \bar{n}\left(x_{2}\right)\right)$ for all pairs $\left(x_{1}, x_{2}\right)$ that we consider (see Fig. 1$)$.
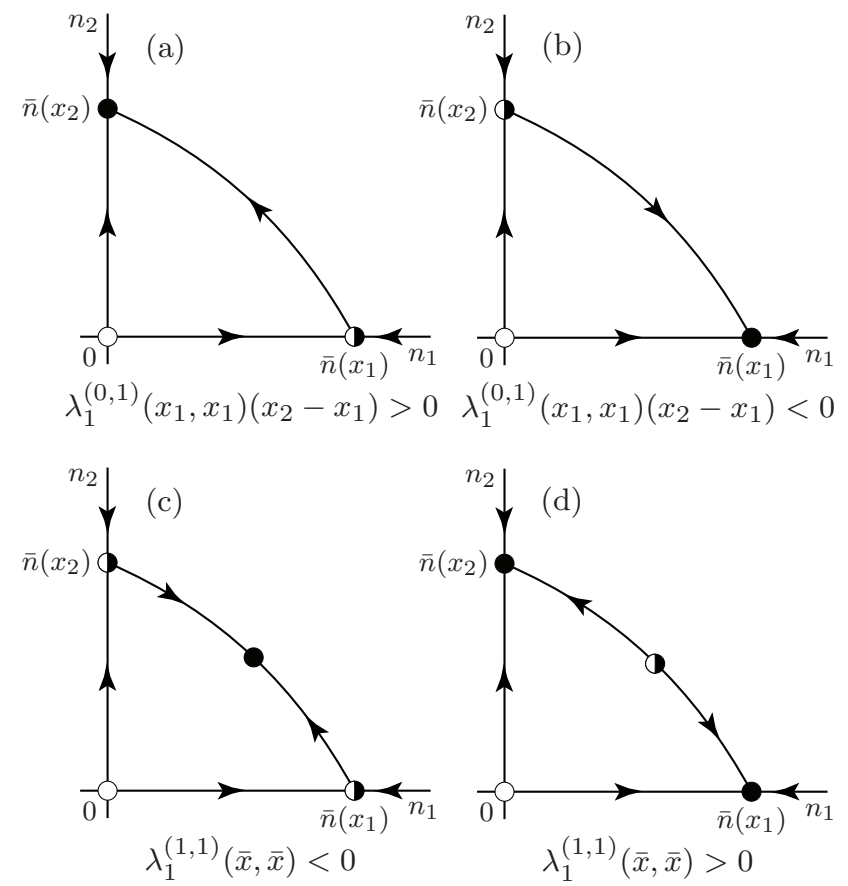

Fig. 1. The four possible resident-mutant competition scenarios for $\left(x_{1}, x_{2}\right)$ close to the singular point $(\bar{x}, \bar{x})$. Mutant dominance (resident substitution) (a) and resident dominance (mutant extinction) (b) away from singularity $\left(\lambda_{1}^{(0,1)}\left(x_{1}, x_{1}\right) \neq 0\right)$ for $x_{2}$ sufficiently close to $x_{1}$. Coexistence (mutant invasion) (c) and mutual exclusion (mutant extinction) (d) for $\left(x_{1}, x_{2}\right)$ sufficiently close to the anti-diagonal $(\bar{x}-\Delta x, \bar{x}+\Delta x), \Delta x$ small. Full points: stable equilibria; half-filled points: saddles; empty points: repellor equilibria.

The monomorphic invasion fitness is the initial (per-capita) growth rate of the mutant population [Metz et al., 1992], i.e.,

$$
\lambda_{1}\left(x, x^{\prime}\right):=g\left(\bar{n}(x), 0, x, x, x^{\prime}\right) \stackrel{P}{=} g_{1}\left(\bar{n}(x), x, x^{\prime}\right),
$$

the resident population settled at equilibrium mutations being rare. Technically, $\lambda_{1}\left(x_{1}, x_{2}\right)$ is the eigenvalue of the monomorphic equilibrium $\left(\bar{n}\left(x_{1}\right), 0\right)$ of model (1) ruling mutant invasion along the eigenvector transversal to the $n_{1}$-axis (Fig. 1 ).

Generically (i.e., if $\lambda_{1}^{(0,1)}(x, x) \neq 0$ [Geritz, 2005, Meszéna et al., 2005, Dercole \& Rinaldi, 2008]), the best performing population wins the competition, so $x$ evolves by small steps in the direction of the selection gradient $\lambda_{1}^{(0,1)}(x, x)$. And in the limit of extremely rare and small mutations, the expected evolutionary dynamics is deterministically described by the $\mathrm{AD}$ canonical equation

$$
\dot{x}=\frac{1}{2} \mu(x) \sigma(x)^{2} \bar{n}(x) \lambda_{1}^{(0,1)}(x, x),
$$

where $\mu(x)$ and $\sigma(x)^{2}$ scale with the frequency and variance of mutations in strategy $x$ (half of which are at disadvantage and go extinct). The strategies annihilating the selection gradient are called singular and correspond to the equilibria of the canonical equation. 


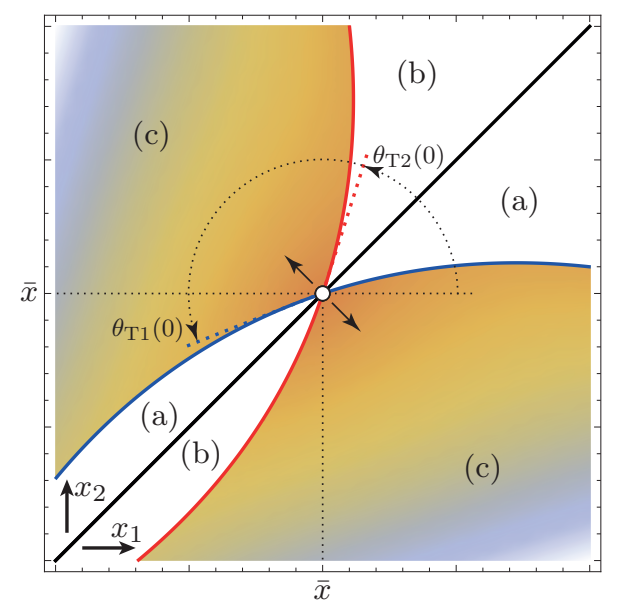

Fig. 2. The resident-mutant coexistence region locally to the singular point $(\bar{x}, \bar{x})$ (shaded area, see scenario (c) in Fig. 1); boundary 1 (blue): transcritical bifurcation of model (1) at which $\bar{n}_{1}\left(x_{1}, x_{2}\right)=0$; boundary 2 (red): transcritical bifurcation of model (1) at which $\bar{n}_{2}\left(x_{1}, x_{2}\right)=0$. For $\left(x_{1}, x_{2}\right)$ in the white areas one of the two populations outcompetes the other (see scenarios (a) and (b) in Fig. 1).

In the vicinity of a singular strategy $\bar{x}$, i.e.,

$$
\lambda_{1}^{(0,1)}(\bar{x}, \bar{x})=0,
$$

the ecological and evolutionary dynamics are dominated by the second derivatives of the monomorphic fitness. In particular, resident-mutant coexistence is possible if

$$
\lambda_{1}^{(1,1)}(\bar{x}, \bar{x})<0 .
$$

2 Geritz et al. $[1997,1998]$ showed that under (G1) resident and mutant mutually invade each other (the instability of the two monomorphic equilibria, see Fig. 1c); and they mutually exclude if the inequality sign in (G1) is reversed (the stability of the two monomorphic equilibria, see Fig. 1d). The uniqueness and stability under (G1) of the internal equilibrium of the resident-mutant model (1) has been later shown (under different ecological settings) in [Geritz, 2005, Meszéna et al., 2005, Dercole \& Geritz, 2015]. When possible, coexistence occurs for $\left(x_{1}, x_{2}\right)$ in a conical region locally to $(\bar{x}, \bar{x})$ (see Fig. 2). The boundaries of the region are transcritical bifurcation curves [Kuznetsov, 2004, Meijer et al., 2009] on which the internal equilibrium collides with one of the monomorphic equilibria (see Sect.2.2). For $\left(x_{1}, x_{2}\right)$ in the coexistence region, we

10 denote the densities of the internal equilibrium with functions $\bar{n}_{1}\left(x_{1}, x_{2}\right)$ and $\bar{n}_{2}\left(x_{1}, x_{2}\right)$, implicitly defined by

$$
\begin{aligned}
& g\left(\bar{n}_{1}\left(x_{1}, x_{2}\right), \bar{n}_{2}\left(x_{1}, x_{2}\right), x_{1}, x_{2}, x_{1}\right)=0, \\
& g\left(\bar{n}_{1}\left(x_{1}, x_{2}\right), \bar{n}_{2}\left(x_{1}, x_{2}\right), x_{1}, x_{2}, x_{2}\right)=0
\end{aligned}
$$

12 (the equilibrium condition for model (1)).

After coexistence evolution is driven by a two-dimensional canonical equation

$$
\dot{x}_{i}=\frac{1}{2} \mu\left(x_{i}\right) \sigma\left(x_{i}\right)^{2} \bar{n}_{i}\left(x_{1}, x_{2}\right) \lambda_{2}^{(0,0,1)}\left(x_{1}, x_{2}, x_{i}\right), \quad i=1,2,
$$

14 where

$$
\lambda_{2}\left(x_{1}, x_{2}, x^{\prime}\right):=g\left(\bar{n}_{1}\left(x_{1}, x_{2}\right), \bar{n}_{2}\left(x_{1}, x_{2}\right), x_{1}, x_{2}, x^{\prime}\right),
$$

is the dimorphic invasion fitness - the initial (per-capita) growth rate of the mutant population of strategy $x^{\prime}$ appeared in an environment set by the two residents $x_{1}$ and $x_{2}$ at their equilibrium densities.

Note the symmetry of the resident-mutant coexistence region w.r.t. the diagonal $x_{1}=x_{2}$ (Fig. 2), that is due to property P3. Indeed, the dynamics of model (1) corresponding to point $\left(x_{1}, x_{2}\right)$ below the diagonal are obtained by those corresponding to point $\left(x_{2}, x_{1}\right)$ above the diagonal by exchanging $n_{1}$ and $n_{2}$, i.e., by exchanging the roles of resident and mutant (hence $\bar{n}_{2}\left(x_{1}, x_{2}\right)=\bar{n}_{1}\left(x_{2}, x_{1}\right)$ ). Consequently, also model $(7)$ 
has a diagonal symmetry - the vector field at $\left(x_{1}, x_{2}\right)$ below the diagonal is obtained by that at $\left(x_{2}, x_{1}\right)$ 2 above the diagonal by exchanging the two components of the field (see the black arrows in Fig. 2).

The (convergence stable) singular strategy $\bar{x}$ is a branching point if the two coexisting similar strategies $4 x_{1}$ and $x_{2}$ tend to diversify according to the dimorphic evolutionary dynamics (7). Technically, this is so if $\dot{x}_{1}(0)<0$ and $\dot{x}_{2}(0)>0$ at a point $\left(x_{1}(0), x_{2}(0)\right)$ of the coexistence region, with $x_{2}(0)>x_{1}(0)$, that 6 is arbitrarily close to $(\bar{x}, \bar{x})$ (see the black arrow above the diagonal in Fig. 2). The singular strategy is a terminal point of the evolutionary dynamics, otherwise.

Geritz et al. [1997, 1998] concluded that a sufficient condition for evolutionary divergence is

$$
\lambda_{1}^{(0,2)}(\bar{x}, \bar{x})>0,
$$

i.e., the condition for evolutionary instability - mutant invasion at $x_{1}=\bar{x}$ [Maynard Smith \& Price, 1973].

The conclusion is based on a second-order Taylor expansion of the dimorphic fitness at $\left(x_{1}, x_{2}, x^{\prime}\right)=(\bar{x}, \bar{x}, \bar{x})$ (see [Geritz et al., 1998] Appendix 1 in particular), that is however nonsmooth there. In fact, by assuming

$\mathrm{C} 1: \lambda_{2}\left(x, x, x^{\prime}\right)=\lambda_{1}\left(x, x^{\prime}\right)$,

the link between the dimorphic and monomorphic fitness functions (induced by properties P1 and P2),

$\mathrm{C} 2: \lambda_{2}\left(x_{1}, x_{2}, x^{\prime}\right)=\lambda_{2}\left(x_{2}, x_{1}, x^{\prime}\right)$,

the order irrelevance of the two residents (property P3)

C3: $\lambda_{2}\left(x_{1}, x_{2}, x_{1}\right)=\lambda_{2}\left(x_{1}, x_{2}, x_{2}\right)=0$,

the resident equilibrium conditions (6),

one gets to nongeneric constraints on the monomorphic fitness, such as $\lambda_{1}^{(2,0)}(\bar{x}, \bar{x})=\lambda_{1}^{(0,2)}(\bar{x}, \bar{x})$ at second order, and similar nonsenses at higher orders (see Appendix 1). In Sect.2.3 we show that the divergence condition (9) is correct, as we rederive it through a proper (radial) expansion of the dimorphic fitness.

In the following we use over-bars to denote evaluations at the singular strategy in the absence of mutants, e.g.

$$
\bar{g}^{(1,0,0,0,0)}:=g^{(1,0,0,0,0)}(\bar{n}(\bar{x}), 0, \bar{x}, \bar{x}, \bar{x}) \stackrel{\mathrm{P} 1}{=} g_{1}^{(1,0,0)}(\bar{n}(\bar{x}), \bar{x}, \bar{x})<0
$$

24 is the stability condition of the resident equilibrium $\bar{n}(\bar{x}), \bar{\lambda}_{1}^{(0,1)}:=\lambda_{1}^{(0,1)}(\bar{x}, \bar{x})=0$ is the singularity condition (5), and $\bar{\lambda}_{1}^{(1,1)}:=\lambda_{1}^{(1,1)}(\bar{x}, \bar{x})$ and $\bar{\lambda}_{1}^{(0,2)}:=\lambda_{1}^{(0,2)}(\bar{x}, \bar{x})$ rule branching at the singular strategy. And 26 from the definition (3) of the monomorphic fitness and property $\mathrm{P} 1,4$, we can write

$$
\begin{aligned}
& \bar{\lambda}_{1}^{(0, q)}=\bar{g}^{(0,0,0,0, q)} \\
& \bar{\lambda}_{1}^{(1, q)}=\bar{g}^{(1,0,0,0, q)} \bar{n}^{(1)}+\bar{\phi}_{1,1}^{(0,0, q)} \bar{n}, \\
& \bar{\lambda}_{1}^{(2, q)}=\bar{g}^{(2,0,0,0, q)}\left(\bar{n}^{(1)}\right)^{2}+2\left(\bar{\phi}_{1,1}^{(0,0, q)}+\bar{\phi}_{1,1}^{(1,0, q)} \bar{n}\right) \bar{n}^{(1)}+\bar{g}^{(1,0,0,0, q)} \bar{n}^{(2)}+\bar{\phi}_{2,1}^{(0,0, q)} \bar{n}+\bar{\phi}_{2,2}^{(0,0, q)} \bar{n}^{2},
\end{aligned}
$$

$q \geq 0$, and so forth (that will be used in Appendix B).

${ }_{28} \quad$ Moreover, taking into account invasion neutrality, i.e.,

$$
\lambda_{1}(x, x)=0
$$

for any $x$ (note that it is nothing but the definition of $\bar{n}(x)$, see Eqs. (2) and (3)), we can avoid the pure so derivatives $\bar{\lambda}_{1}^{(d, 0)}, d \geq 1$, since by the $x$-derivatives of $(12)$ at $(\bar{x}, \bar{x})$ we have

$$
\begin{aligned}
& \bar{\lambda}_{1}^{(1,0)}+\bar{\lambda}_{1}^{(0,1)}=0, \\
& \bar{\lambda}_{1}^{(2,0)}+2 \bar{\lambda}_{1}^{(1,1)}+\bar{\lambda}_{1}^{(0,2)}=0, \\
& \bar{\lambda}_{1}^{(3,0)}+3 \bar{\lambda}_{1}^{(2,1)}+3 \bar{\lambda}_{1}^{(1,2)}+\bar{\lambda}_{1}^{(0,3)}=0,
\end{aligned}
$$

and so forth. 
Finally, recalling our aim of studying the branching bifurcation at which $\bar{\lambda}_{1}^{(0,2)}=0$ under (G1), we can 2 always assume

$$
\bar{\lambda}_{1}^{(1,1)}+\bar{\lambda}_{1}^{(0,2)} \stackrel{(13 \mathrm{~b})}{=} \frac{1}{2}\left(\bar{\lambda}_{1}^{(0,2)}-\bar{\lambda}_{1}^{(2,0)}\right)<0 .
$$

The quantity in (14) is the $x$-derivative of the selection gradient $\lambda_{1}^{(0,1)}(x, x)$ at $\bar{x}$, so its negative sign gives 4 the (convergence) stability of the singular strategy. Condition (14) thus prevents $\bar{x}$ to be involved in a bifurcation of the monomorphic canonical equation (4) in the vicinity of the branching bifurcation [Dercole 6 \& Geritz, 2015].

\subsection{Expansion of the resident-mutant coexistence region}

8 The equilibrium $\left(\bar{n}_{1}\left(x_{1}, x_{2}\right), \bar{n}_{2}\left(x_{1}, x_{2}\right)\right)$ of model (1), at which the two similar strategies (the resident and the mutant) coexist during the initial phase of branching, is defined by Eqs. $(6 \mathrm{a}, \mathrm{b})$, where $x_{1}$ and $x_{2}$ play 10 the role of parameters. Under the genericity condition (G1), Dercole \& Geritz [2015] showed that the coexistence equilibrium can only undergo transcritical bifurcations in the vicinity of point $\left(x_{1}, x_{2}\right)=(\bar{x}, \bar{x})$

12 in the strategy plane, $\bar{x}$ being a singular strategy. Due to the symmetry of model (1) w.r.t. the diagonal $x_{1}=x_{2}$, the diagonal itself is a (degenerate) transcritical bifurcation at which the segment $n_{1}+n_{2}=\bar{n}\left(x_{1}\right)$

14 is composed of a continuum of (critically) stable equilibria. Crossing the diagonal far from the singularity causes the switch of stability between the two monomorphic equilibria $\left(\bar{n}\left(x_{1}\right), 0\right)$ and $\left(0, \bar{n}\left(x_{2}\right)\right)$. Moreover, 16 two (standard) transcritical bifurcations are rooted at point $(\bar{x}, \bar{x})$ and constitute the boundaries of the resident-mutant coexistence region (see Figs. 1 and 2).

${ }_{18}$ The transcritical bifurcation at which the coexistence equilibrium collides with the monomorphic one at $\left(\bar{n}\left(x_{1}\right), 0\right)$ is defined by

$$
\lambda_{1}\left(x_{1}, x_{2}\right)=0,
$$

20 i.e., a zero eigenvalue associated to the direction of mutant invasion (recall the text below definition (3)). As done in [Dercole \& Geritz, 2015], to geometrically characterize the bifurcation curve, it is convenient

22 to use the polar coordinates $(\varepsilon, \theta)$,

$$
x_{1}:=\bar{x}+\varepsilon \cos \theta, \quad x_{2}:=\bar{x}+\varepsilon \sin \theta,
$$

and to $\varepsilon$-parameterize the curve as $\theta=\theta_{\mathrm{T} 2}(\varepsilon)$. Along the curve the mutant density $\bar{n}_{2}\left(x_{1}, x_{2}\right)$ is zero, so ${ }_{24}$ we call this boundary of the coexistence region "boundary 2." Then

$$
\lambda_{1}\left(\bar{x}+\varepsilon \cos \theta_{\mathrm{T} 2}(\varepsilon), \bar{x}+\varepsilon \sin \theta_{\mathrm{T} 2}(\varepsilon)\right)=0
$$

is an identity for any (sufficiently small) $\varepsilon \geq 0$, and by evaluating Eq. (16) and its $\varepsilon$-derivatives at $\varepsilon=0$ we

${ }_{26}$ can solve the resulting expressions for $\theta_{\mathrm{T} 2}(0)$ and the derivatives $\theta_{\mathrm{T} 2}^{(k)}(0), k \geq 1$. The angle $\theta_{\mathrm{T} 2}(0)$ gives the tangent direction to the bifurcation curve at $\varepsilon=0$, while the first nonvanishing derivative $\theta_{\mathrm{T} 2}^{(k)}(0)$ determines

28 the curvature (whether $\theta$ increases or decreases by moving away from $\varepsilon=0$ in the $\theta_{\mathrm{T} 2}(0)$-direction, see Fig. 2).

30 Specifically, taking into account the singularity condition (5) (and the properties in (13)), the first $\varepsilon$-derivative of Eq.(16) at $\varepsilon=0$ is an identity, whereas the second and third derivatives give

$$
\left(\sin \theta_{\mathrm{T} 2}(0)-\cos \theta_{\mathrm{T} 2}(0)\right)\left(2 \cos \theta_{\mathrm{T} 2}(0) \bar{\lambda}_{1}^{(1,1)}+\left(\sin \theta_{\mathrm{T} 2}(0)+\cos \theta_{\mathrm{T} 2}(0)\right) \bar{\lambda}_{1}^{(0,2)}\right)=0
$$

32 and

$$
\begin{array}{r}
6\left(\left(\sin ^{2} \theta_{\mathrm{T} 2}(0)-2 \sin \theta_{\mathrm{T} 2}(0) \cos \theta_{\mathrm{T} 2}(0)-\cos ^{2} \theta_{\mathrm{T} 2}(0)\right) \bar{\lambda}_{1}^{(1,1)}-2 \sin \theta_{\mathrm{T} 2}(0) \cos \theta_{\mathrm{T} 2}(0) \bar{\lambda}_{1}^{(0,2)}\right) \theta_{\mathrm{T} 2}^{(1)}(0)= \\
\left(\sin \theta_{\mathrm{T} 2}(0)-\cos \theta_{\mathrm{T} 2}(0)\right) \times \\
\left(3 \cos ^{2} \theta_{\mathrm{T} 2}(0) \bar{\lambda}_{1}^{(2,1)}+3 \cos \theta_{\mathrm{T} 2}(0)\left(\sin \theta_{\mathrm{T} 2}(0)+\cos \theta_{\mathrm{T} 2}(0)\right) \bar{\lambda}_{1}^{(1,2)}+\left(1+\sin \theta_{\mathrm{T} 2}(0) \cos \theta_{\mathrm{T} 2}(0)\right) \bar{\lambda}_{1}^{(0,3)}\right)
\end{array}
$$

(see Supplementary Material, last section). From the $\left(\sin \theta_{\mathrm{T} 2}(0)-\cos \theta_{\mathrm{T} 2}(0)\right)$ factor in the left- and right34 hand sides of (17a) and (17b), we have the solutions $\theta_{\mathrm{T} 2}(0)=\frac{1}{4} \pi$ and $\frac{5}{4} \pi, \theta_{\mathrm{T} 2}^{(1)}(0)=0$, which correspond 
to the diagonal $x_{1}=x_{2}$ (for which $\theta_{\mathrm{T} 2}^{(k)}(0)=0$ for all $k \geq 1$ ), whereas solving the second factor in (17a) we 2 obtain

$$
\tan \theta_{\mathrm{T} 2}(0)=-\frac{2 \bar{\lambda}_{1}^{(1,1)}+\bar{\lambda}_{1}^{(0,2)}}{\bar{\lambda}_{1}^{(0,2)}} \stackrel{(13 \mathrm{~b})}{=} \frac{\bar{\lambda}_{1}^{(2,0)}}{\bar{\lambda}_{1}^{(0,2)}} .
$$

Note that two solutions for $\theta_{\mathrm{T} 2}(0)$ can be considered from Eq. (18), one above and one below the diagonal. ${ }_{4}$ We can in fact assume $\tan \theta_{\mathrm{T} 2}(0) \neq 1$ under $(14)$.

Substituting the solution for $\theta_{\mathrm{T} 2}(0)$ above the diagonal into (17b) and solving for $\theta_{\mathrm{T} 2}^{(1)}(0)$, we get

$$
\theta_{\mathrm{T} 2}^{(1)}(0)=-\frac{4\left(\bar{\lambda}_{1}^{(1,1)}\right)^{2} \bar{\lambda}_{1}^{(0,3)}-2 \bar{\lambda}_{1}^{(1,1)} \bar{\lambda}_{1}^{(0,2)}\left(3 \bar{\lambda}_{1}^{(1,2)}-\bar{\lambda}_{1}^{(0,3)}\right)+\left(\bar{\lambda}_{1}^{(0,2)}\right)^{2}\left(3 \bar{\lambda}_{1}^{(2,1)}+\bar{\lambda}_{1}^{(0,3)}\right)}{6 \sqrt{2}\left(2\left(\bar{\lambda}_{1}^{(1,1)}\right)^{2}+2 \bar{\lambda}_{1}^{(1,1)} \bar{\lambda}_{1}^{(0,2)}+\left(\bar{\lambda}_{1}^{(0,2)}\right)^{2}\right)^{3 / 2}},
$$

6 whereas taking $\theta_{\mathrm{T} 2}(0)$ below the diagonal one gets opposite curvature (see Supplementary Material). Moreover, the higher-order curvatures for $\theta_{\mathrm{T} 2}(0)$ above/below the diagonal are same/opposite for even/odd $\left.{ }_{8} \quad k \geq 2\right)$. This allows us to keep the solution above the diagonal and use the expansion $\theta_{\mathrm{T} 2}(\varepsilon)=$ $\theta_{\mathrm{T} 2}(0)+\theta_{\mathrm{T} 2}^{(1)}(0) \varepsilon+\cdots+\theta_{\mathrm{T} 2}^{(k)}(0) \varepsilon^{k}+O\left(\varepsilon^{k+1}\right)$ also for negative $\varepsilon$ to describe both branches (above and below the diagonal) of the bifurcation curve. In the rest of the paper, if not otherwise specified, we therefore consider angles above the diagonal and restrict our attention to

$$
\frac{1}{4} \pi<\theta<\frac{5}{4} \pi
$$

12 and

$$
x_{2}-x_{1}=\varepsilon(\sin \theta-\cos \theta)>0 .
$$

The bifurcation curve corresponding to the standard transcritical at the monomorphic equilibrium ${ }_{14}\left(0, \bar{n}\left(x_{2}\right)\right)$ is symmetric w.r.t. the diagonal to the one occurring at $\left(\bar{n}\left(x_{1}\right), 0\right)$ and is $\varepsilon$-parameterized as $\theta=\theta_{\mathrm{T} 1}(\varepsilon)$ (see Fig. 2). This is "boundary 1" of the resident-mutant coexistence region, along which the 16 resident density $\bar{n}_{1}\left(x_{1}, x_{2}\right)$ is zero. It is indeed defined by $\lambda_{1}\left(x_{2}, x_{1}\right)=0$, i.e., a zero eigenvalue at $\left(0, \bar{n}\left(x_{2}\right)\right)$ associated to the direction of "resident invasion." As a result, $\tan \theta_{\mathrm{T} 1}(0)$ is the inverse of the expression in 18 (18), i.e.,

$$
\tan \theta_{\mathrm{T} 1}(0)=\frac{\bar{\lambda}_{1}^{(0,2)}}{\bar{\lambda}_{1}^{(2,0)}}
$$

and the derivative $\theta_{\mathrm{T} 1}^{(k)}(0)$ coincides with $\theta_{\mathrm{T} 2}^{(k)}(0)$ or with its opposite for odd/even $k \geq 1$, i.e.,

$$
\theta_{\mathrm{T} 1}^{(k)}(0)=(-1)^{k-1} \theta_{\mathrm{T} 2}^{(k)}(0) .
$$

The two (standard) transcritical bifurcation curves define the resident-mutant coexistence region. That is, $\bar{n}_{i}\left(x_{1}, x_{2}\right)=0$ along boundary $i$ defined by the curve $\theta=\theta_{\mathrm{T} i}(\varepsilon), i=1,2$. In Fig. 2 the boundaries are first-order approximated by $\theta_{\mathrm{T} i}(\varepsilon)=\theta_{\mathrm{T} i}(0)+\theta_{\mathrm{T} i}^{(1)}(0) \varepsilon$ for small (positive and negative) $\varepsilon$. Locally to $(\bar{x}, \bar{x})$, the coexistence region is a cone spanned by the rays within angles $\theta_{\mathrm{T} 2}(0)<\theta_{\mathrm{T} 1}(0)$ (see (18) and (21), where $\left|\bar{\lambda}_{1}^{(0,2)}\right|<\left|\bar{\lambda}_{1}^{(2,0)}\right|$ due to (14)). At the bifurcation $\left(\bar{\lambda}_{1}^{(0,2)}=0\right)$, the tangent directions to the boundaries 1 and 2 are respectively horizontal $\left(\theta_{\mathrm{T} 1}(0)=\pi\right)$ and vertical $\left(\theta_{\mathrm{T} 2}(0)=\frac{1}{2} \pi\right)$, and the curvature is dominated by $\bar{\lambda}_{1}^{(0,3)}$ (see (19) with $\bar{\lambda}_{1}^{(0,2)}=0$ under (G1)). Generically, we assume

$$
\bar{\lambda}_{1}^{(0,3)} \neq 0
$$




\subsection{Expansion of the dimorphic invasion fitness}

2 As anticipated at the end of Sect.2.1, the dimorphic invasion fitness $\lambda_{2}\left(x_{1}, x_{2}, x^{\prime}\right)$ cannot be Taylor expanded at $\left(x_{1}, x_{2}, x^{\prime}\right)=(\bar{x}, \bar{x}, \bar{x})$. This is due to the nonsmoothness of the resident-mutant coexistence ${ }_{4}$ equilibrium $\left(\bar{n}_{1}\left(x_{1}, x_{2}\right), \bar{n}_{2}\left(x_{1}, x_{2}\right)\right)$, e.g. $\bar{n}_{1}\left(x_{1}, x_{2}\right)$ approaches $\bar{n}(\bar{x})$ along boundary 2 of the coexistence region, being zero along boundary 1 . The key observation, made in [Durinx, 2008] and (more explicitly) in

$6 \quad$ [Dercole \& Geritz, 2015], is that the equilibrium densities $\bar{n}_{1}\left(x_{1}, x_{2}\right)$ and $\bar{n}_{2}\left(x_{1}, x_{2}\right)$ can be smoothly defined at $(\bar{x}, \bar{x})$ along each ray in the cone of coexistence, $\left(x_{1}, x_{2}\right)=(\bar{x}+\varepsilon \cos \theta, \bar{x}+\varepsilon \sin \theta), \theta \in\left[\theta_{\mathrm{T} 2}(0), \theta_{\mathrm{T} 1}(0)\right]$, the result being $\theta$-dependent (actually, any $\theta$ in the interval (20a) can be used, though either $\bar{n}_{1}\left(x_{1}, x_{2}\right.$ ) or $\bar{n}_{2}\left(x_{1}, x_{2}\right)$ is negative outside the cone).

Specifically, Dercole \& Geritz [2015] made use of new variables (following Meszéna et al. [2005] and Dercole \& Rinaldi [2008]), the sum of the resident densities $s:=n_{1}+n_{2}$ and the relative mutant density $r:=n_{2} /\left(n_{1}+n_{2}\right)$ (the inverse transformation giving $n_{1}=(1-r) s$ and $n_{2}=r s$ ), and exploited their fast-slow nature for small $\varepsilon$. At constant $r, s$ quickly converges to the fast-equilibrium manifold $\left\{s_{f}(r, \varepsilon, \theta), r \in[0,1]\right\}$ connecting the two monomorphic equilibria (see the internal trajectories in Fig. 1), so the slow dynamics of $r$ can be studied in isolation by restricting $n_{1}$ and $n_{2}$ to $(1-r) s_{f}(r, \varepsilon, \theta)$ and $r s_{f}(r, \varepsilon, \theta)$.

From the resident-mutant model (1), the fast-equilibrium manifold is defined by

$$
\begin{aligned}
0 & =\dot{s}=\dot{n}_{1}+\dot{n}_{2} \\
& =(1-r) g\left((1-r) s_{f}(r, \varepsilon, \theta), r s_{f}(r, \varepsilon, \theta), \bar{x}+\varepsilon \cos \theta, \bar{x}+\varepsilon \sin \theta, \bar{x}+\varepsilon \cos \theta\right) \\
& +r g\left((1-r) s_{f}(r, \varepsilon, \theta), r s_{f}(r, \varepsilon, \theta), \bar{x}+\varepsilon \cos \theta, \bar{x}+\varepsilon \sin \theta, \bar{x}+\varepsilon \sin \theta\right),
\end{aligned}
$$

whereas the slow dynamics of $r$ is ruled by

$$
\begin{aligned}
\dot{r} & =r(1-r) g\left((1-r) s_{f}(r, \varepsilon, \theta), r s_{f}(r, \varepsilon, \theta), \bar{x}+\varepsilon \cos \theta, \bar{x}+\varepsilon \sin \theta, \bar{x}+\varepsilon \sin \theta\right) \\
& -r(1-r) g\left((1-r) s_{f}(r, \varepsilon, \theta), r s_{f}(r, \varepsilon, \theta), \bar{x}+\varepsilon \cos \theta, \bar{x}+\varepsilon \sin \theta, \bar{x}+\varepsilon \cos \theta\right) .
\end{aligned}
$$

${ }_{18}$ The equilibrium solutions for $r$ are $r=0$ and $r=1$, corresponding to the monomorphic equilibria of model (1), together with the solution $\bar{r}(\varepsilon, \theta) \in[0,1]$ of

$$
\begin{aligned}
0 & =g\left((1-\bar{r}(\varepsilon, \theta)) s_{f}(\bar{r}(\varepsilon, \theta), \varepsilon, \theta), \bar{r}(\varepsilon, \theta) s_{f}(\bar{r}(\varepsilon, \theta), \varepsilon, \theta), \bar{x}+\varepsilon \cos \theta, \bar{x}+\varepsilon \sin \theta, \bar{x}+\varepsilon \sin \theta\right) \\
& -g\left((1-\bar{r}(\varepsilon, \theta)) s_{f}(\bar{r}(\varepsilon, \theta), \varepsilon, \theta), \bar{r}(\varepsilon, \theta) s_{f}(\bar{r}(\varepsilon, \theta), \varepsilon, \theta), \bar{x}+\varepsilon \cos \theta, \bar{x}+\varepsilon \sin \theta, \bar{x}+\varepsilon \cos \theta\right),
\end{aligned}
$$

that is unique under (G1) [Dercole \& Geritz, 2015].

The equilibrium densities $\bar{n}_{1}\left(x_{1}, x_{2}\right)$ and $\bar{n}_{2}\left(x_{1}, x_{2}\right)$ can then be rewritten in terms of $(\varepsilon, \theta)$ as

$$
\bar{n}_{1}(\varepsilon, \theta)=(1-\bar{r}(\varepsilon, \theta)) s_{f}(\bar{r}(\varepsilon, \theta), \varepsilon, \theta), \quad \bar{n}_{2}(\varepsilon, \theta)=\bar{r}(\varepsilon, \theta) s_{f}(\bar{r}(\varepsilon, \theta), \varepsilon, \theta),
$$

and can be evaluated also at $\varepsilon=0$ for any given $\theta$ in the cone of coexistence (see Tabs. 1 and 2, first row).

The dimorphic fitness can also be rewritten in $(\varepsilon, \theta)$ and $\Delta x^{\prime}:=x^{\prime}-\bar{x}$ as

$$
\lambda_{2}\left(\varepsilon, \theta, \Delta x^{\prime}\right):=g\left((1-\bar{r}(\varepsilon, \theta)) s_{f}(\bar{r}(\varepsilon, \theta), \varepsilon, \theta), \bar{r}(\varepsilon, \theta) s_{f}(\bar{r}(\varepsilon, \theta), \varepsilon, \theta), \bar{x}+\varepsilon \cos \theta, \bar{x}+\varepsilon \sin \theta, \bar{x}+\Delta x^{\prime}\right),
$$

and can be Taylor expanded around $\left(\varepsilon, \Delta x^{\prime}\right)=(0,0)$ at given $\theta$. We proceed up to third order. This involves up to third $\varepsilon$-derivatives of the fast-equilibrium manifold $s_{f}$, whereas only the first $\varepsilon$-derivative of the slow equilibrium $\bar{r}$ is involved.

The required zero- and higher-order terms of the fast-equilibrium manifold (w.r.t. $\varepsilon$ and mixed $(r, \varepsilon))$ are reported in Tab. 1, whereas those of the slow equilibrium are in Tab. 2. They are obtained by differentiating Eqs. (23) and (24) at $\varepsilon=0$ and solving for the unknown terms. Note in particular the expression of $\bar{r}(0, \theta)$

30 (Tab.2, first row), which goes from zero to one when $\theta$ moves from $\theta_{\mathrm{T} 2}(0)$ to $\theta_{\mathrm{T} 1}(0)$, i.e., from one extreme to the other of the cone of coexistence, passing through $\frac{1}{2}$ when $\theta=\frac{3}{4} \pi$.

${ }_{32}$ The $\varepsilon$-derivatives $s_{f}^{(0, k, 0)}(r, 0, \theta), k \geq 1$, characterize the $\varepsilon$-perturbations of the fast-equilibrium manifold from the zero-order solution $s_{f}(r, 0, \theta)=\bar{n}(\bar{x})$. Note that they are polynomial expressions in $r$ with degree equal to the order of differentiation and coefficients that are ultimately functions of the singular strategy $\bar{x}$ and of the perturbation direction $\theta$. This is due to property $\mathrm{P} 4$, where $n_{1}+n_{2}$ becomes $\bar{n}(\bar{x})$ ${ }_{36}$ when $\varepsilon \rightarrow 0$, while $n_{1}^{i_{1}} n_{2}^{i_{2}}$ becomes $(1-r)^{i_{1}} r^{i_{2}} \bar{n}(\bar{x})^{i_{1}+i_{2}}$. The mixed derivatives of $s_{f}$ characterize joint $(r, \varepsilon)$-perturbations, i.e., involving both changes in the shape of the manifold and movements along it. 
Table 1. $\varepsilon$-expansion of the fast-equilibrium manifold $\left\{s_{f}(r, \varepsilon, \theta), r \in[0,1]\right\}$.

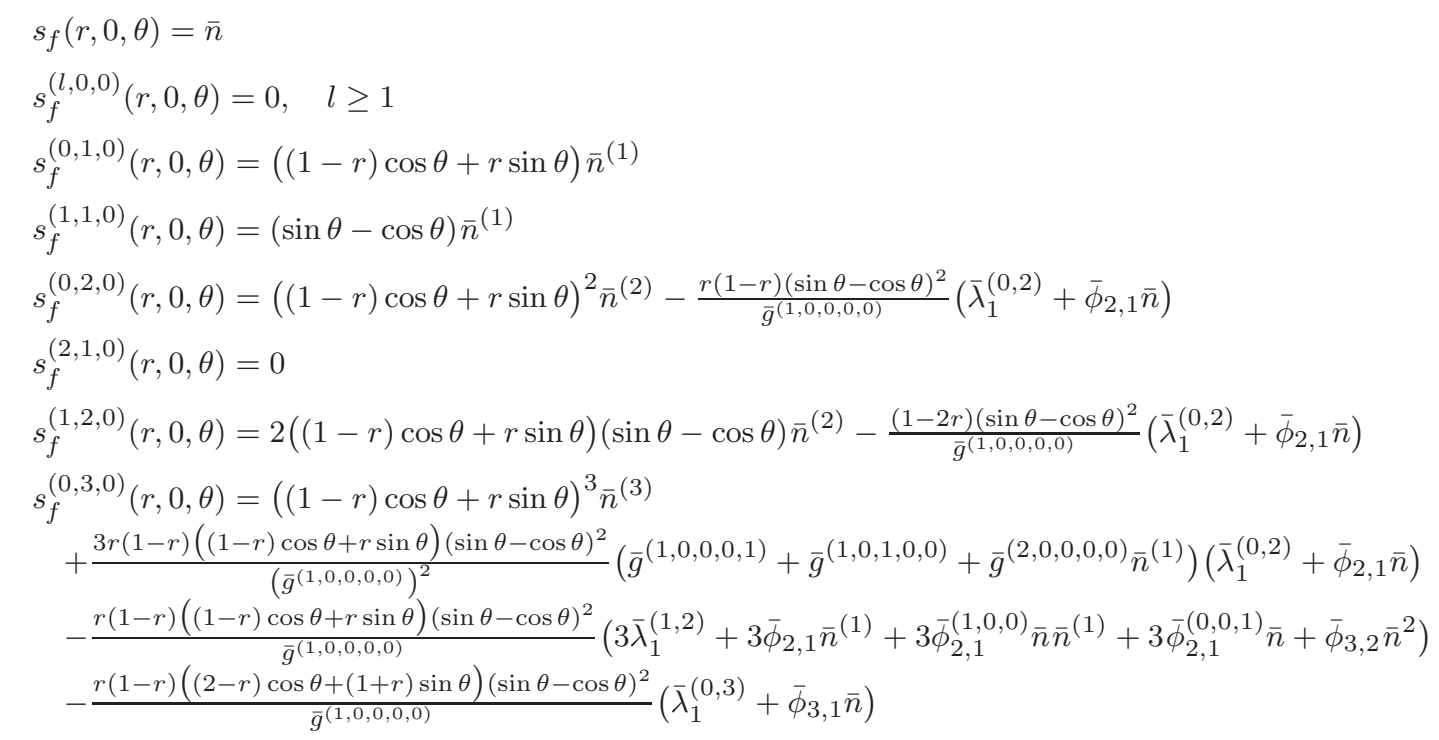

Table 2. $\varepsilon$-expansion of the slow equilibrium $\bar{r}(\varepsilon, \theta)$.

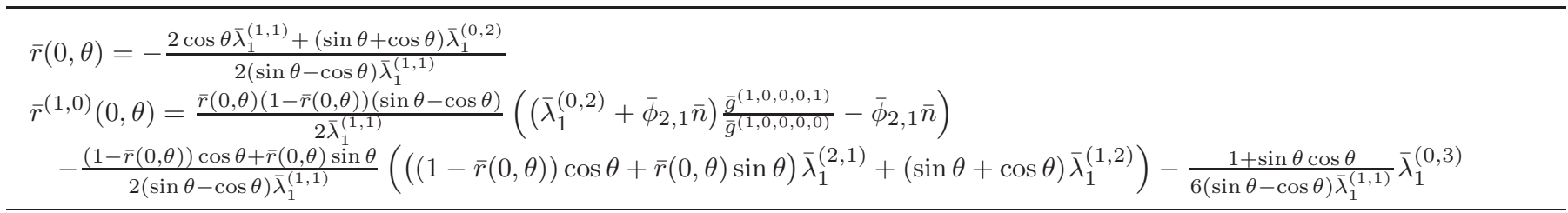

Table 3. $\left(\varepsilon, \Delta x^{\prime}\right)$-expansion of the dimorphic fitness.

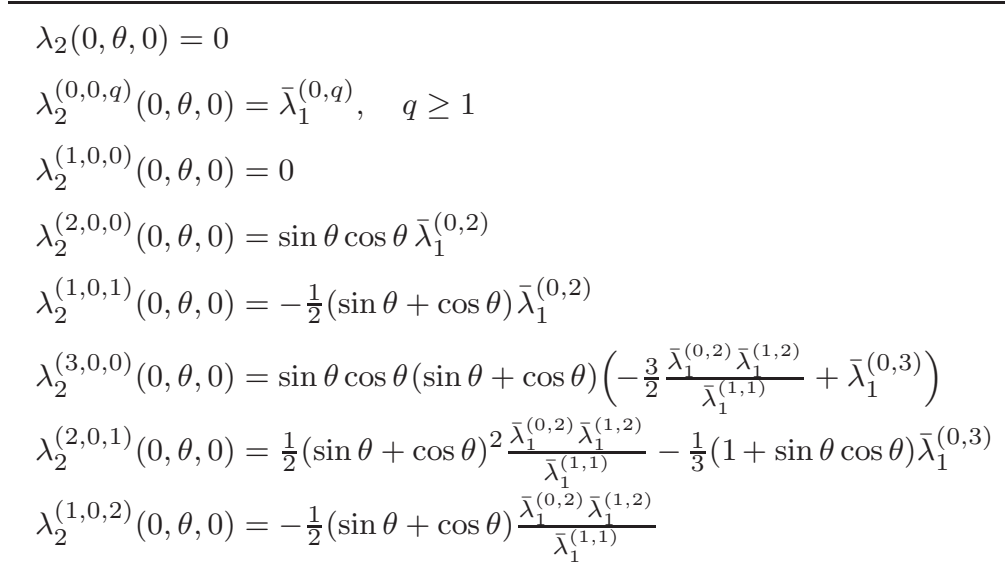

Note that they can be simply obtained by $r$-differentiating $s_{f}^{(0, k, 0)}(r, 0, \theta)$. The $\varepsilon$-derivatives $\bar{r}^{(k, 0)}(0, \theta)$, $2 k \geq 1$, describe how the coexistence equilibrium $(25)$ moves from $((1-\bar{r}(0, \theta)) \bar{n}(\bar{x}), \bar{r}(0, \theta) \bar{n}(\bar{x}))$ along the fast-equilibrium manifold when $\varepsilon$ is perturbed in the direction $\theta$.

That the third derivative $\bar{r}^{(3,0)}(0, \theta)$ of the slow equilibrium is not needed in the cubic $\varepsilon$-expansion of the dimorphic fitness is easy to note. In fact, if one of the $\bar{r}$ 's in front of $s_{f}$ in $(26)$ is $\varepsilon$-differentiated 6 three times, then no differentiation is taken w.r.t. the $\varepsilon$ in $x_{1}$ and $x_{2}$ (the third and fourth arguments of the $g$-function), so at $x_{1}=x_{2}=\bar{x}$ the densities $n_{1}$ and $n_{2}$ (at first and second arguments) sum up due 8 to property P2 and the $\bar{r}$ 's in front of $s_{f}$ play no role. And also the third $\varepsilon$-derivatives of the $\bar{r}$ 's at first argument of $s_{f}$ do not appear, since they are multiplied by the first $r$-derivative of $s_{f}$ that vanishes with

${ }_{10} \varepsilon$ (see Tab. 1, second row), the fast-equilibrium manifold becoming the straight segment $n_{1}+n_{2}=\bar{n}(\bar{x})$ as $\varepsilon \rightarrow 0$. More intricate to see is that also the second derivative of the slow equilibrium is not needed. The 
derivative $\bar{r}^{(2,0)}(0, \theta)$ indeed appears in the cubic $\varepsilon$-term in (26) and is multiplied by

$$
\left.3\left(s_{f}^{(1,1,0)}(r, 0, \theta) \bar{g}^{(1,0,0,0,0)}+\bar{n} \sin \theta\left(g^{(0,1,0,1,0)}-g^{(1,0,0,1,0)}\right)-\bar{n} \cos \theta\left(g^{(1,0,1,0,0)}-g^{(0,1,1,0,0)}\right)\right)\right|_{\varepsilon=0, \Delta x^{\prime}=0},
$$

2 where the arguments of the $g$-derivatives with no over-bar are as in Eq. (26) (checked in the Supplementary Material, last section). All of the terms in (27) are generically nonzero, however, their sum vanishes thanks

4 again to property P4. This can be explicitly seen in Appendix B, substituting (B.9a-d) and (B.12) into (27) and noting the expression for $s_{f}^{(1,1,0)}(r, 0, \theta)$ from Tab. 1.

Taking the results in Tabs. 1 and 2 into account, exploiting the properties P1-P4 of Sect.2.1, and assuming genericity (G1), the dimorphic fitness derivatives appearing in the third-order $\varepsilon$-expansion

$$
\begin{aligned}
\lambda_{2}\left(\varepsilon, \theta, \Delta x^{\prime}\right) & =\bar{\lambda}_{2}+\bar{\lambda}_{2}^{(1,0,0)} \varepsilon+\bar{\lambda}_{2}^{(0,0,1)} \Delta x^{\prime}+ \\
& +\frac{1}{2} \bar{\lambda}_{2}^{(2,0,0)} \varepsilon^{2}+\bar{\lambda}_{2}^{(1,0,1)} \varepsilon \Delta x^{\prime}+\frac{1}{2} \bar{\lambda}_{2}^{(0,0,2)}\left(\Delta x^{\prime}\right)^{2}+ \\
& +\frac{1}{6} \bar{\lambda}_{2}^{(3,0,0)} \varepsilon^{3}+\frac{1}{2} \bar{\lambda}_{2}^{(2,0,1)} \varepsilon^{2} \Delta x^{\prime}+\frac{1}{2} \bar{\lambda}_{2}^{(1,0,2)} \varepsilon\left(\Delta x^{\prime}\right)^{2}+\frac{1}{6} \bar{\lambda}_{2}^{(0,0,3)}\left(\Delta x^{\prime}\right)^{3}+O\left(\left\|\left(\varepsilon, \Delta x^{\prime}\right)\right\|^{4}\right)
\end{aligned}
$$

(over-bars here denote evaluations at $\left.\left(\varepsilon, \Delta x^{\prime}\right)=(0,0)\right)$ result as in Tab. 3. Rewriting the dimorphic fitness back in terms of the resident and mutant strategies $\left(\bar{x}+\Delta x_{1}, \bar{x}+\Delta x_{2}, \bar{x}+\Delta x^{\prime}\right), \Delta x_{i}:=x_{i}-\bar{x}, i=1,2$, i.e., recalling the definition (15) of the polar coordinates $(\varepsilon, \theta)$, we then come to the following third-order approximation:

$$
\begin{aligned}
& \tilde{\lambda}_{2}\left(\Delta x_{1}, \Delta x_{2}, \Delta x^{\prime}\right):= \\
& \quad\left(\frac{1}{2} \bar{\lambda}_{1}^{(0,2)}-\frac{1}{4} \frac{\bar{\lambda}_{1}^{(0,2)} \bar{\lambda}_{1}^{(1,2)}}{\bar{\lambda}_{1}^{(1,1)}}\left(\Delta x_{1}+\Delta x_{2}\right)+\frac{1}{6} \bar{\lambda}_{1}^{(0,3)}\left(\Delta x_{1}+\Delta x_{2}+\Delta x^{\prime}\right)\right)\left(\Delta x^{\prime}-\Delta x_{1}\right)\left(\Delta x^{\prime}-\Delta x_{2}\right) .
\end{aligned}
$$

8 Recall that the right-hand side in (29) is an expansion taken at given $\theta \in\left[\theta_{\mathrm{T} 2}(0), \theta_{\mathrm{T} 1}(0)\right]$ around $\left(\varepsilon, \Delta x^{\prime}\right)=$ $(0,0)$ (the higher-order terms are indeed $O\left(\left\|\left(\varepsilon, \Delta x^{\prime}\right)\right\|^{4}\right)$ ), and not an expansion w.r.t. $\left(x_{1}, x_{2}, x^{\prime}\right)$ around $(\bar{x}, \bar{x}, \bar{x})$. It can nevertheless be used as an approximation of the dimorphic fitness $(8)$ for $\left(x_{1}, x_{2}\right)$ in the resident-mutant coexistence region locally to $(\bar{x}, \bar{x})$ and $x^{\prime}$ close to $\bar{x}$.

12 Interestingly, the second-order term in (29) coincides with that obtained by Geritz et al. [1997, 1998] assuming a smooth dimorphic fitness (see Eq. A10 in [Geritz et al., 1998]; the zero- and first-order terms 14 vanish at the singular point $\left.\left(x_{1}, x_{2}, x^{\prime}\right)=(\bar{x}, \bar{x}, \bar{x})\right)$. Thus, the (second-order) branching condition (9) of Geritz et al. [1997, 1998] is correct, though assuming smoothness implies senseless constraints on the monomorphic fitness derivatives (starting with the second-order, see Appendix 1).

To illustrate our approach at work, the reader can see Appendix B, where we compute step by step all terms in the expansion (28) up to second order. The computation of the third-order terms can be checked in the Supplementary Material.

\section{The normal form of the branching bifurcation}

From (7) and from the analysis in Sects. 2.2 and 2.3, we now derive two simplified models that approximate the dimorphic evolutionary dynamics locally to a branching point. In the first model, we take into account the curvature of the boundaries of the resident-mutant coexistence region, to preserve the geometric features relating the evolutionary trajectories with the boundaries themselves. The curvature of the boundaries is however irrelevant for the branching bifurcation $\left(\bar{\lambda}_{1}^{(0,2)}=0\right.$ under $\left.(\mathrm{G} 1)\right)$, so we ignore it in the second model, that we propose as the normal form for the dimorphic canonical equation (7) at the incipient branching.

First we get rid of the scaling $\frac{1}{2} \mu\left(x_{i}\right) \sigma\left(x_{i}\right)^{2}, i=1,2$. Locally to $(\bar{x}, \bar{x})$ this can be done in two steps. A near-identity coordinate transformation, $z_{1,2}=z_{1,2}\left(x_{1}, x_{2}\right)\left(\partial z_{i} / \partial x_{j}=1\right.$ if $i=j, 0$ otherwise), whose expansion can be set to eliminate all the derivatives of $\mu$ and $\sigma$ in the expansion of the scaling terms around $x_{1}=x_{2}=\bar{x}$; a time-scaling $\tau=\frac{1}{2} \mu(\bar{x}) \sigma(\bar{x})^{2} t, \tau$ being the new time. For simplicity, we keep on using variables $x_{i}$, actually $\Delta x_{i}, i=1,2$, and $t$ for the new variables and time. 
Second, we use our radial expansion to approximate the coexistence equilibrium (25). The expansion 2 is done up to first order (in $\varepsilon$ ), i.e.,

$$
\begin{aligned}
& \tilde{n}_{1}(\varepsilon, \theta):=(1-\bar{r}(0, \theta)) \bar{n}+\varepsilon\left((1-\bar{r}(0, \theta))((1-\bar{r}(0, \theta)) \cos \theta+\bar{r}(0, \theta) \sin \theta) \bar{n}^{(1)}-\bar{r}^{(1,0)}(0, \theta) \bar{n}\right)= \\
& \tilde{n}_{1}\left(\Delta x_{1}, \Delta x_{2}\right):=-\frac{\bar{\lambda}_{1}^{(1,1)} \Delta x_{2}+\frac{1}{2} \bar{\lambda}_{1}^{(0,2)}\left(\Delta x_{1}+\Delta x_{2}\right)}{\left(\Delta x_{1}-\Delta x_{2}\right) \bar{\lambda}_{1}^{(1,1)}} \bar{n}-\frac{1}{24\left(\Delta x_{1}-\Delta x_{2}\right)\left(\bar{\lambda}_{1}^{(1,1)}\right)^{3}} \times \\
& \left(4 \bar{\lambda}_{1}^{(0,3)}\left(\bar{\lambda}_{1}^{(1,1)}\right)^{2} \bar{n}\left(\Delta x_{1}^{2}+\Delta x_{1} \Delta x_{2}+\Delta x_{2}^{2}\right)\right. \\
& -6 \bar{\lambda}_{1}^{(0,2)} \bar{\lambda}_{1}^{(1,1)} \bar{n}^{(1)}\left(\Delta x_{1}+\Delta x_{2}\right)\left(2 \bar{\lambda}_{1}^{(1,1)} \Delta x_{2}+\bar{\lambda}_{1}^{(0,2)}\left(\Delta x_{1}+\Delta x_{2}\right)\right) \\
& +3 \bar{n} \bar{\lambda}_{1}^{(0,2)}\left(-2 \bar{\lambda}_{1}^{(1,1)} \bar{\lambda}_{1}^{(1,2)}+\bar{\lambda}_{1}^{(0,2)} \bar{\lambda}_{1}^{(2,1)}\right)\left(\Delta x_{1}+\Delta x_{2}\right)^{2} \\
& +3 \bar{n}\left(2 \bar{\lambda}_{1}^{(1,1)} \Delta x_{1}+\bar{\lambda}_{1}^{(0,2)}\left(\Delta x_{1}+\Delta x_{2}\right)\right)\left(2 \bar{\lambda}_{1}^{(1,1)} \Delta x_{2}+\bar{\lambda}_{1}^{(0,2)}\left(\Delta x_{1}+\Delta x_{2}\right)\right) \times \\
& \left.\left(\bar{\phi}_{2,1}^{(1,0,0)} \bar{n}+\frac{\bar{g}^{(1,0,0,0,1)}}{\bar{g}^{(1,0,0,0,0)}}\left(\bar{\lambda}_{1}^{(0,2)}-\bar{\phi}_{2,1} \bar{n}\right)\right)\right) \\
& \tilde{n}_{2}(\varepsilon, \theta):=\bar{r}(0, \theta) \bar{n}+\varepsilon\left(\bar{r}(0, \theta)((1-\bar{r}(0, \theta)) \cos \theta+\bar{r}(0, \theta) \sin \theta) \bar{n}^{(1)}+\bar{r}^{(1,0)}(0, \theta) \bar{n}\right)= \\
& \tilde{n}_{2}\left(\Delta x_{1}, \Delta x_{2}\right):=-\frac{\bar{\lambda}_{1}^{(1,1)} \Delta x_{1}+\frac{1}{2} \bar{\lambda}_{1}^{(0,2)}\left(\Delta x_{1}+\Delta x_{2}\right)}{\left(\Delta x_{2}-\Delta x_{1}\right) \bar{\lambda}_{1}^{(1,1)}} \bar{n}-\frac{1}{24\left(\Delta x_{2}-\Delta x_{1}\right)\left(\bar{\lambda}_{1}^{(1,1)}\right)^{3}} \times \\
& \left(4 \bar{\lambda}_{1}^{(0,3)}\left(\bar{\lambda}_{1}^{(1,1)}\right)^{2} \bar{n}\left(\Delta x_{1}^{2}+\Delta x_{1} \Delta x_{2}+\Delta x_{2}^{2}\right)\right. \\
& -6 \bar{\lambda}_{1}^{(0,2)} \bar{\lambda}_{1}^{(1,1)} \bar{n}^{(1)}\left(\Delta x_{1}+\Delta x_{2}\right)\left(2 \bar{\lambda}_{1}^{(1,1)} \Delta x_{1}+\bar{\lambda}_{1}^{(0,2)}\left(\Delta x_{1}+\Delta x_{2}\right)\right) \\
& +3 \bar{n} \bar{\lambda}_{1}^{(0,2)}\left(-2 \bar{\lambda}_{1}^{(1,1)} \bar{\lambda}_{1}^{(1,2)}+\bar{\lambda}_{1}^{(0,2)} \bar{\lambda}_{1}^{(2,1)}\right)\left(\Delta x_{1}+\Delta x_{2}\right)^{2} \\
& +3 \bar{n}\left(2 \bar{\lambda}_{1}^{(1,1)} \Delta x_{1}+\bar{\lambda}_{1}^{(0,2)}\left(\Delta x_{1}+\Delta x_{2}\right)\right)\left(2 \bar{\lambda}_{1}^{(1,1)} \Delta x_{2}+\bar{\lambda}_{1}^{(0,2)}\left(\Delta x_{1}+\Delta x_{2}\right)\right) \times \\
& \left.\left(\bar{\phi}_{2,1}^{(1,0,0)} \bar{n}+\frac{\bar{g}^{(1,0,0,0,1)}}{\bar{g}^{(1,0,0,0,0)}}\left(\bar{\lambda}_{1}^{(0,2)}-\bar{\phi}_{2,1} \bar{n}\right)\right)\right)
\end{aligned}
$$

4 because this is consistent with our approximation, locally to $(\bar{x}, \bar{x})$, of the resident-mutant coexistence region (Sect.2.2), where, indeed, up to third-order derivatives of the monomorphic fitness are involved. Moreover, 6 defining the function $\theta_{\mathrm{T} i}(\varepsilon)$ of the coexistence region boundary $i$ by $\tilde{n}_{i}\left(\varepsilon, \theta_{\mathrm{T} i}(\varepsilon)\right)=0$, we checked that one obtains for $\theta_{\mathrm{T} i}(0)$ and $\theta_{\mathrm{T} i}^{(1)}(0), i=1,2$, the same results derived in Sect. 2.2 (see Eqs. (18), (19), (21),

8 and $(22))$. Note that the symmetry $\tilde{n}_{1}\left(\Delta x_{2}, \Delta x_{1}\right)=\tilde{n}_{2}\left(\Delta x_{1}, \Delta x_{2}\right)$ is preserved by the approximation. Also note the term $\left(\Delta x_{i}-\Delta x_{j}\right), i \neq j$, at denominator of $\tilde{n}_{i}\left(\Delta x_{1}, \Delta x_{2}\right)$, which makes evident the nonsmoothness 10 of functions $\bar{n}_{1}\left(x_{1}, x_{2}\right)$ and $\bar{n}_{2}\left(x_{1}, x_{2}\right)$ at $(\bar{x}, \bar{x})$.

Instead of approximating $\bar{n}_{i}\left(\bar{x}+\Delta x_{1}, \bar{x}+\Delta x_{2}\right)$ with the complicated $\tilde{n}_{i}\left(\Delta x_{1}, \Delta x_{2}\right)$ in $(30), i=1,2$, we note that the following expressions share the same structure of the linear terms at numerator:

$$
\begin{array}{r}
\eta_{1}\left(\Delta x_{1}, \Delta x_{2}\right):=\bar{\lambda}_{1}^{(1,1)} \Delta x_{2}+\frac{1}{2} \bar{\lambda}_{1}^{(0,2)}\left(\Delta x_{1}+\Delta x_{2}\right)+\frac{1}{2} \bar{\lambda}_{1}^{(1,2)} \Delta x_{2}\left(\Delta x_{1}+\Delta x_{2}\right)+\frac{1}{2} \bar{\lambda}_{1}^{(2,1)} \Delta x_{2}^{2} \\
+\frac{1}{6} \bar{\lambda}_{1}^{(0,3)}\left(\Delta x_{1}^{2}+\Delta x_{1} \Delta x_{2}+\Delta x_{2}^{2}\right), \\
\eta_{2}\left(\Delta x_{1}, \Delta x_{2}\right):=\bar{\lambda}_{1}^{(1,1)} \Delta x_{1}+\frac{1}{2} \bar{\lambda}_{1}^{(0,2)}\left(\Delta x_{1}+\Delta x_{2}\right)+\frac{1}{2} \bar{\lambda}_{1}^{(1,2)} \Delta x_{1}\left(\Delta x_{1}+\Delta x_{2}\right)+\frac{1}{2} \bar{\lambda}_{1}^{(2,1)} \Delta x_{1}^{2} \\
+\frac{1}{6} \bar{\lambda}_{1}^{(0,3)}\left(\Delta x_{1}^{2}+\Delta x_{1} \Delta x_{2}+\Delta x_{2}^{2}\right) .
\end{array}
$$

The expressions in (31) come from the cubic expansion w.r.t. $\left(x_{1}, x_{2}\right)$ of $\lambda_{1}\left(x_{2}, x_{1}\right)$ and $\lambda_{1}\left(x_{1}, x_{2}\right)$, respec14 tively. Specifically,

$$
\begin{aligned}
& \lambda_{1}\left(\bar{x}+\Delta x_{2}, \bar{x}+\Delta x_{1}\right)=\eta_{1}\left(\Delta x_{1}, \Delta x_{2}\right)\left(\Delta x_{1}-\Delta x_{2}\right)+O\left(\left\|\left(\Delta x_{1}, \Delta x_{2}\right)\right\|^{4}\right), \\
& \lambda_{1}\left(\bar{x}+\Delta x_{1}, \bar{x}+\Delta x_{2}\right)=\eta_{2}\left(\Delta x_{1}, \Delta x_{2}\right)\left(\Delta x_{2}-\Delta x_{1}\right)+O\left(\left\|\left(\Delta x_{1}, \Delta x_{2}\right)\right\|^{4}\right),
\end{aligned}
$$


so $\eta_{i}\left(\Delta x_{1}, \Delta x_{2}\right)=0$ is a quadratic approximation (in the variables $\left(\Delta x_{1}, \Delta x_{2}\right)$ ) of the boundary $i$ of the coexistence region. It is a different approximation w.r.t. $\theta_{\mathrm{T} i}(\varepsilon)=\theta_{\mathrm{T} i}(0)+\theta_{\mathrm{T} i}^{(1)}(0) \varepsilon$, derived in Sect. 2.2, and w.r.t. $\tilde{n}_{i}\left(\varepsilon, \theta_{\mathrm{T} i}(\varepsilon)\right)=0$ proposed above. But again, defining the function $\theta_{\mathrm{T} i}(\varepsilon)$ by ${ }_{4} \eta_{i}\left(\varepsilon \cos \theta_{\mathrm{T} i}(\varepsilon), \varepsilon \sin \theta_{\mathrm{T} i}(\varepsilon)\right)=0$, we checked that one obtains for $\theta_{\mathrm{T} i}(0)$ and $\theta_{\mathrm{T} i}^{(1)}(0), i=1,2$, the results of Sect.2.2.

6 By means of another near-identity coordinate transformation and another time-scaling $(\tau=$ $\left.-\bar{n} / \bar{\lambda}_{1}^{(1,1)} t\right)$, we can then replace $\bar{n}_{i}\left(\bar{x}+\Delta x_{1}, \bar{x}+\Delta x_{2}\right)$ in the dimorphic canonical equation (7) with

$$
\tilde{n}_{i}\left(\Delta x_{1}, \Delta x_{2}\right):=\frac{\eta_{i}\left(\Delta x_{1}, \Delta x_{2}\right)}{\Delta x_{i}-\Delta x_{j}}, \quad i \neq j,
$$

8 and use $\eta_{i}\left(\Delta x_{1}, \Delta x_{2}\right)=0$ as an approximation of the coexistence region boundary $i, i=1,2$. Note that the new $\tilde{n}_{1}\left(\Delta x_{1}, \Delta x_{2}\right)$ and $\tilde{n}_{2}\left(\Delta x_{1}, \Delta x_{2}\right)$ are positive, by construction, inside the approximated coexistence o region (easy to check, e.g. above the diagonal where $0<\Delta x_{2}=-\Delta x_{1}$ ).

Third step, we compute the selection gradients $\lambda_{2}^{(0,0,1)}\left(\bar{x}+\Delta x_{1}, \bar{x}+\Delta x_{2}, \bar{x}+\Delta x_{i}\right), i=1,2$, using our approximation (29), thus obtaining

$$
\tilde{\lambda}_{2}^{(0,0,1)}\left(\Delta x_{1}, \Delta x_{2}, \Delta x_{i}\right):=s_{i}\left(\Delta x_{1}, \Delta x_{2}\right)\left(\Delta x_{i}-\Delta x_{j}\right), \quad i \neq j
$$

with

$$
\begin{aligned}
& s_{1}\left(\Delta x_{1}, \Delta x_{2}\right):=\left(\frac{1}{2} \bar{\lambda}_{1}^{(0,2)}-\frac{1}{4} \frac{\bar{\lambda}_{1}^{(0,2)} \bar{\lambda}_{1}^{(1,2)}}{\bar{\lambda}_{1}^{(1,1)}}\left(\Delta x_{1}+\Delta x_{2}\right)+\frac{1}{6} \bar{\lambda}_{1}^{(0,3)}\left(2 \Delta x_{1}+\Delta x_{2}\right)\right), \\
& s_{2}\left(\Delta x_{1}, \Delta x_{2}\right):=\left(\frac{1}{2} \bar{\lambda}_{1}^{(0,2)}-\frac{1}{4} \frac{\bar{\lambda}_{1}^{(0,2)} \bar{\lambda}_{1}^{(1,2)}}{\bar{\lambda}_{1}^{(1,1)}}\left(\Delta x_{1}+\Delta x_{2}\right)+\frac{1}{6} \bar{\lambda}_{1}^{(0,3)}\left(\Delta x_{1}+2 \Delta x_{2}\right)\right) .
\end{aligned}
$$

Our first simplified model - the one taking the curvatures $\theta_{\mathrm{T} 1}^{(1)}(0)$ and $\theta_{\mathrm{T} 2}^{(1)}(0)$ into account - then reads

$$
\dot{x}_{i}=\tilde{n}_{i}\left(\Delta x_{1}, \Delta x_{2}\right) \tilde{\lambda}_{2}^{(0,0,1)}\left(\Delta x_{1}, \Delta x_{2}, \Delta x_{i}\right)=\eta_{i}\left(\Delta x_{1}, \Delta x_{2}\right) s_{i}\left(\Delta x_{1}, \Delta x_{2}\right), \quad i=1,2 .
$$

Note the simplification of the differences $\left(\Delta x_{i}-\Delta x_{j}\right)$ at denominator in definition (32) and at numerator in (33), that makes the model equations polynomial (and therefore smooth!).

Our second model is the most simple form showing the bifurcation, so we call it the "normal form" (though we do not provide a formal proof of the topological equivalence with the dimorphic canonical equation (7)). It considers only a conical coexistence region $\theta \in\left[\theta_{\mathrm{T} 2}(0), \theta_{\mathrm{T} 1}(0)\right]$ and, consistently, a zeroorder approximation (in $\varepsilon$ ) of the coexistence equilibrium (25), i.e.,

$$
\begin{aligned}
\tilde{n}_{1}(\varepsilon, \theta) & :=(1-\bar{r}(0, \theta)) \bar{n}= \\
\tilde{n}_{1}\left(\Delta x_{1}, \Delta x_{2}\right) & :=\frac{\eta_{1}\left(\Delta x_{1}, \Delta x_{2}\right)}{\Delta x_{1}-\Delta x_{2}}, \\
\tilde{n}_{2}(\varepsilon, \theta) & :=\bar{r}(0, \theta) \bar{n}= \\
\tilde{n}_{2}\left(\Delta x_{1}, \Delta x_{2}\right) & :=\frac{\eta_{2}\left(\Delta x_{1}, \Delta x_{2}\right)}{\Delta x_{2}-\Delta x_{1}},
\end{aligned}
$$

with

$$
\begin{aligned}
& \eta_{1}\left(\Delta x_{1}, \Delta x_{2}\right):=\bar{\lambda}_{1}^{(1,1)} \Delta x_{2}+\frac{1}{2} \bar{\lambda}_{1}^{(0,2)}\left(\Delta x_{1}+\Delta x_{2}\right), \\
& \eta_{2}\left(\Delta x_{1}, \Delta x_{2}\right):=\bar{\lambda}_{1}^{(1,1)} \Delta x_{1}+\frac{1}{2} \bar{\lambda}_{1}^{(0,2)}\left(\Delta x_{1}+\Delta x_{2}\right) .
\end{aligned}
$$

22 The model equations are formally those in (35), but with the new definitions of $\tilde{n}_{i}\left(\Delta x_{1}, \Delta x_{2}\right)$ and $\eta_{i}\left(\Delta x_{1}, \Delta x_{2}\right), i=1,2$, in (36) and (37).

The unfolding parameter - that we move across zero - is $\bar{\lambda}_{1}^{(0,2)}$. Three other parameters are left in the normal form $(34,35,37): \bar{\lambda}_{1}^{(1,1)}, \bar{\lambda}_{1}^{(0,3)}$, and $\bar{\lambda}_{1}^{(1,2)}$. The first two are constrained by the genericity conditions ${ }_{26}$ (G1) and (G2), whereas $\bar{\lambda}_{1}^{(1,2)}$ plays no role at the bifurcation, as it only appears multiplied by $\bar{\lambda}_{1}^{(0,2)}$ in 
$s_{i}\left(\Delta x_{1}, \Delta x_{2}\right)$ (definition (34)). Only the product $\bar{\lambda}_{1}^{(1,1)} \bar{\lambda}_{1}^{(0,3)}$ is relevant at the bifurcation $\left(\bar{\lambda}_{1}^{(0,2)}=0\right)$. Being $2 \bar{\lambda}_{1}^{(1,1)}$ constrained in sign, $\bar{\lambda}_{1}^{(0,3)}$ is the only relevant coefficient of the normal form.

Finally, it is important to remark that none of the two models $(31,34,35)$ and $(34,35,37)$ correspond 4 to an $\varepsilon$-expansion of the dimorphic canonical equation (7). E.g., the first model includes cubic $\varepsilon$-termscomposed of the product of a linear term in $\bar{n}_{i}(\varepsilon, \theta)$ with a quadratic term in $\lambda_{2}^{(0,0,1)}\left(\varepsilon, \theta, \Delta x_{i}\right)$-but misses others. This is due to the choice of separately $\varepsilon$-expanding $\bar{n}_{i}\left(x_{1}, x_{2}\right)$ and $\lambda_{2}^{(0,0,1)}\left(x_{1}, x_{2}, x_{i}\right)$ in Eq. (7), with the advantage of preserving some structural features of the canonical equation, e.g., the presence of

boundary equilibria when $\tilde{n}_{i}\left(\Delta x_{1}, \Delta x_{2}\right)$ and $\tilde{\lambda}_{2}^{(0,0,1)}\left(\Delta x_{1}, \Delta x_{2}, \Delta x_{j}\right)$ vanish with $i \neq j$ (see next section).

\section{The unfolding of the bifurcation}

Under the genericity conditions (G1) and (G2), we analyze in this section the dynamics of the branching bifurcation normal form, i.e., model $(34,35,37)$ restricted to the cone of resident-mutant coexistence ${ }_{12} \tilde{n}_{i}\left(\Delta x_{1}, \Delta x_{2}\right) \geq 0, i=1,2$, defined in $(36,37)$, by varying the parameter $\bar{\lambda}_{1}^{(0,2)}$ across zero.

By inspection of Eqs. $(34,35,37)$, it is straightforward to check that there are four equilibria:

${ }_{14}$ E0: $\left(\Delta \bar{x}_{1}, \Delta \bar{x}_{2}\right)=(0,0)$, at which $\eta_{1}\left(\Delta \bar{x}_{1}, \Delta \bar{x}_{2}\right)=\eta_{2}\left(\Delta \bar{x}_{1}, \Delta \bar{x}_{2}\right)=0$.

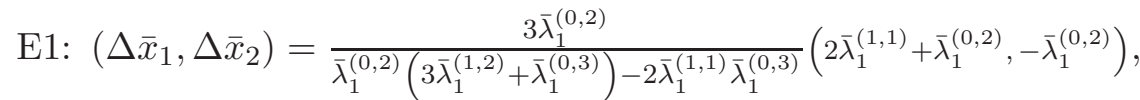
annihilating $\eta_{1}\left(\Delta \bar{x}_{1}, \Delta \bar{x}_{2}\right)$ and $s_{2}\left(\Delta \bar{x}_{1}, \Delta \bar{x}_{2}\right)$.

$\mathrm{E} 2:\left(\Delta \bar{x}_{1}, \Delta \bar{x}_{2}\right)=\frac{3 \bar{\lambda}_{1}^{(0,2)}}{\bar{\lambda}_{1}^{(0,2)}\left(3 \bar{\lambda}_{1}^{(1,2)}+\bar{\lambda}_{1}^{(0,3)}\right)-2 \bar{\lambda}_{1}^{(1,1)} \bar{\lambda}_{1}^{(0,3)}}\left(-\bar{\lambda}_{1}^{(0,2)}, 2 \bar{\lambda}_{1}^{(1,1)}+\bar{\lambda}_{1}^{(0,2)}\right)$, annihilating $\eta_{2}\left(\Delta \bar{x}_{1}, \Delta \bar{x}_{2}\right)$ and $s_{1}\left(\Delta \bar{x}_{1}, \Delta \bar{x}_{2}\right)$.

E3: $\left(\Delta \bar{x}_{1}, \Delta \bar{x}_{2}\right)=\left(-\frac{\bar{\lambda}_{1}^{(1,1)} \bar{\lambda}_{1}^{(0,2)}}{\bar{\lambda}_{1}^{(1,1)} \bar{\lambda}_{1}^{(0,3)}-\bar{\lambda}_{1}^{(0,2)} \bar{\lambda}_{1}^{(1,2)}}, \Delta \bar{x}_{1}\right)$, at which $s_{1}\left(\Delta \bar{x}_{1}, \Delta \bar{x}_{2}\right)=s_{2}\left(\Delta \bar{x}_{1}, \Delta \bar{x}_{2}\right)=0$.

Note that the Jacobian of model $(34,35,37)$ at E0 is given by

$$
\frac{1}{2} \bar{\lambda}_{1}^{(0,2)}\left[\begin{array}{cc}
\frac{1}{2} \bar{\lambda}_{1}^{(0,2)} & \bar{\lambda}_{1}^{(1,1)}+\frac{1}{2} \bar{\lambda}_{1}^{(0,2)} \\
\bar{\lambda}_{1}^{(1,1)}+\frac{1}{2} \bar{\lambda}_{1}^{(0,2)} & \frac{1}{2} \bar{\lambda}_{1}^{(0,2)}
\end{array}\right],
$$

so the diagonal $\Delta x_{1}=\Delta x_{2}$ and the anti-diagonal $\Delta x_{1}+\Delta x_{2}=0$ are the eigenvectors respectively associated 22 to the eigenvalues $\frac{1}{2} \bar{\lambda}_{1}^{(0,2)}\left(\bar{\lambda}_{1}^{(1,1)}+\bar{\lambda}_{1}^{(0,2)}\right)$ and $-\frac{1}{2} \bar{\lambda}_{1}^{(1,1)} \bar{\lambda}_{1}^{(0,2)}$, for $\bar{\lambda}_{1}^{(0,2)} \neq 0$. Further note that E1 and E2 are symmetric boundary equilibria respectively lying on the coexistence cone boundaries 1 and 2 , on which $\tilde{n}_{1}\left(\Delta x_{1}, \Delta x_{2}\right)$ and $\tilde{n}_{2}\left(\Delta x_{1}, \Delta x_{2}\right)$ vanish, whereas E3 lies on the diagonal and is therefore not feasible for the dimorphic canonical equation $(7)$.

The four equilibria are all involved in the bifurcation occurring at $\bar{\lambda}_{1}^{(0,2)}=0$, as they collide at $(0,0)$ at the bifurcation. Under the genericity conditions (G1) and (G2), equilibria E0-3 intersect transversely as $\bar{\lambda}_{1}^{(0,2)}$ moves across zero. The bifurcation classifies as a non-simple branch point [Govaerts, 2000, Meijer et al., 2009] (not to be confused with the branching point of AD!), i.e., the transversal intersection of more than two $\bar{\lambda}_{1}^{(0,2)}$-parameterized equilibrium branches. This bifurcation generically requires the continuation problem [Allgower \& Georg, 1990] defining the intersecting equilibrium branches to have a nullspace with 32 dimension larger than two at the bifurcation. Specifically for our case, the continuation problem is defined in the space $\left(\Delta \bar{x}_{1}, \Delta \bar{x}_{2}, \bar{\lambda}_{1}^{(0,2)}\right)$ by

$$
C\left(\Delta \bar{x}_{1}, \Delta \bar{x}_{2}, \bar{\lambda}_{1}^{(0,2)}\right):=\left(C_{1}\left(\Delta \bar{x}_{1}, \Delta \bar{x}_{2}, \bar{\lambda}_{1}^{(0,2)}\right), C_{2}\left(\Delta \bar{x}_{1}, \Delta \bar{x}_{2}, \bar{\lambda}_{1}^{(0,2)}\right)\right)=0
$$

34 with

$$
C_{i}\left(\Delta \bar{x}_{1}, \Delta \bar{x}_{2}, \bar{\lambda}_{1}^{(0,2)}\right):=\eta_{i}\left(\Delta x_{1}, \Delta x_{2}, \bar{\lambda}_{1}^{(0,2)}\right) s_{i}\left(\Delta x_{1}, \Delta x_{2}, \bar{\lambda}_{1}^{(0,2)}\right), \quad i=1,2,
$$



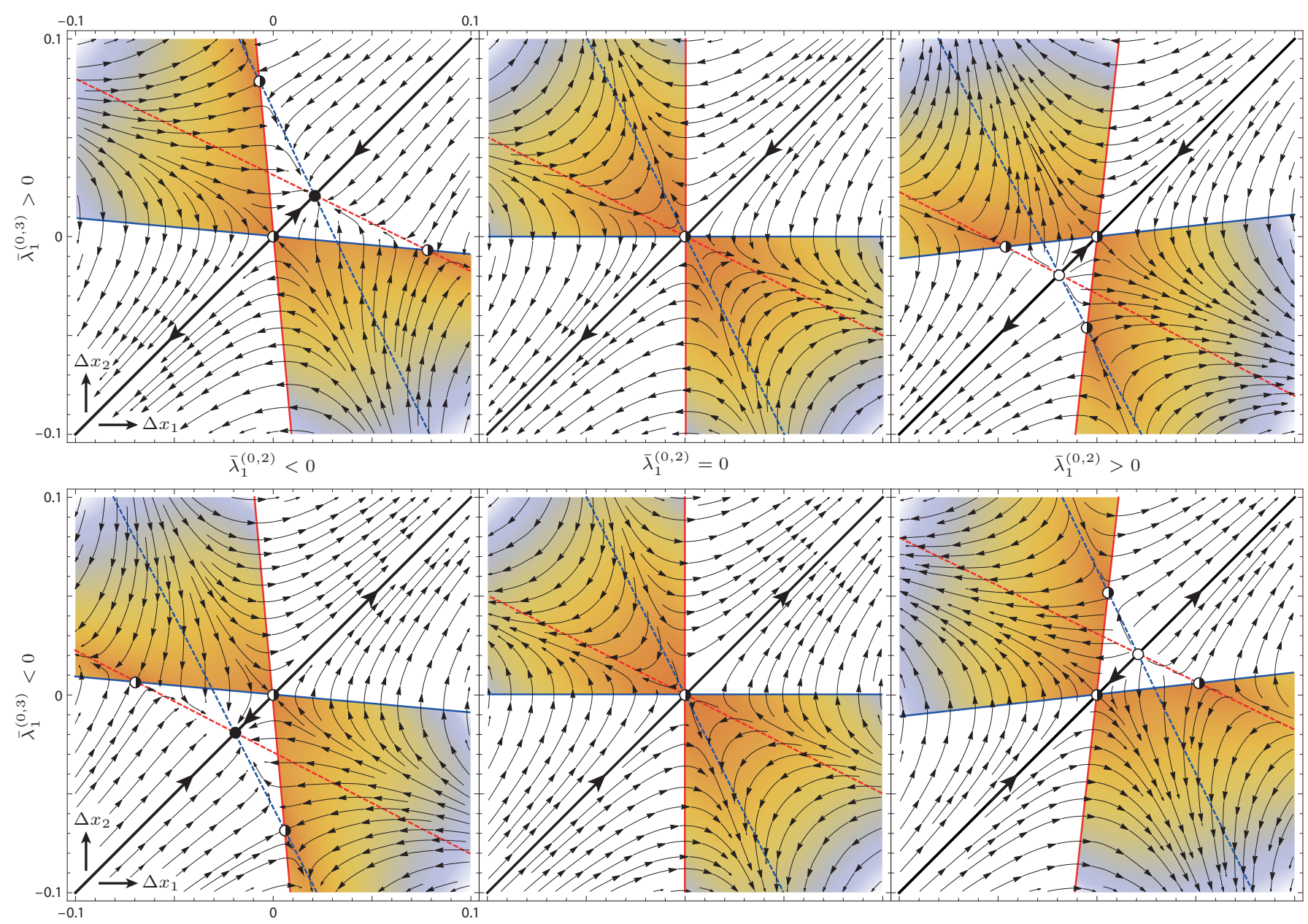

Fig. 3. Unfolding of the branching bifurcation using model $(34,35,37)$. The unfolding parameter $\bar{\lambda}_{1}^{(0,2)}$ increases from left to right and vanishes in the central panel, in which all equilibria E0-E3 collide at $\left(\Delta x_{1}, \Delta x_{2}\right)=(0,0)$. Top/bottom rows: $\bar{\lambda}_{1}^{(0,3)} \gtrless 0$. The resident-mutant coexistence region is shaded, with color code orange-to-blue measuring the magnitude of the vector field. The region boundary $1\left(\eta_{1}\left(\Delta x_{1}, \Delta x_{2}\right)=0(37)\right)$ and the internal $x_{1}$-nullcline $\left(s_{1}\left(\Delta x_{1}, \Delta x_{2}\right)=0(34)\right)$ are plotted in blue (solid and dashed); red for boundary $2\left(\eta_{2}\left(\Delta x_{1}, \Delta x_{2}\right)=0(37)\right)$ and the internal $x_{2}$-nullcline $\left(s_{2}\left(\Delta x_{1}, \Delta x_{2}\right)=0(34)\right)$. Full points: stable equilibria; half-filled points: saddles; empty points: repellor equilibria.

where $\bar{\lambda}_{1}^{(0,2)}$ is explicitly mentioned as an argument of functions $\eta_{i}$ and $s_{i}$. The Jacobian of function $C$ w.r.t. $\left(\Delta \bar{x}_{1}, \Delta \bar{x}_{2}, \bar{\lambda}_{1}^{(0,2)}\right)$ is indeed a $(2 \times 3)$ null matrix at the bifurcation (easy to check), i.e., the nullspace is three-dimensional. Due to the symmetries of the dimorphic canonical equation, this bifurcation can occur as a codimension-one, i.e., moving a single model parameter (see [Govaerts, 2000], Sect.8.2).

Two cases can be distinguished, namely $\bar{\lambda}_{1}^{(0,3)}>0$ and $\bar{\lambda}_{1}^{(0,3)}<0$, whose unfoldings are pictured in

6 Fig. 3 (top and bottom panels, respectively). The movements and stability of the four equilibria, as $\bar{\lambda}_{1}^{(0,2)}$ goes from negative to positive, are evident from the graphics (left-to-right panels). In particular, the flow of model $(34,35,37)$ is drawn also outside the resident-mutant coexistence cone to make stability easily readable. Note that the stability for the unrestricted model is different from the stability for the dimorphic 10 canonical equation. E.g., equilibrium E0 is always unstable (saddle type) for the unrestricted model, though is stable/unstable for the dimorphic canonical equation when $\bar{\lambda}_{1}^{(0,2)} \lessgtr 0$ (evolutionary stability/branching).

Also note that the two cases $\left(\bar{\lambda}_{1}^{(0,3)} \gtrless 0\right)$ are topologically equivalent (at the bifurcation there is a symmetry w.r.t. the anti-diagonal $\Delta x_{1}+\Delta x_{2}=0$ ), so their distinction is mathematically irrelevant. However, the distinction is biologically important and becomes evident if one considers the curvature of the boundaries of the resident-mutant coexistence region, as we do in model $(31,34,35)$. The unfoldings 

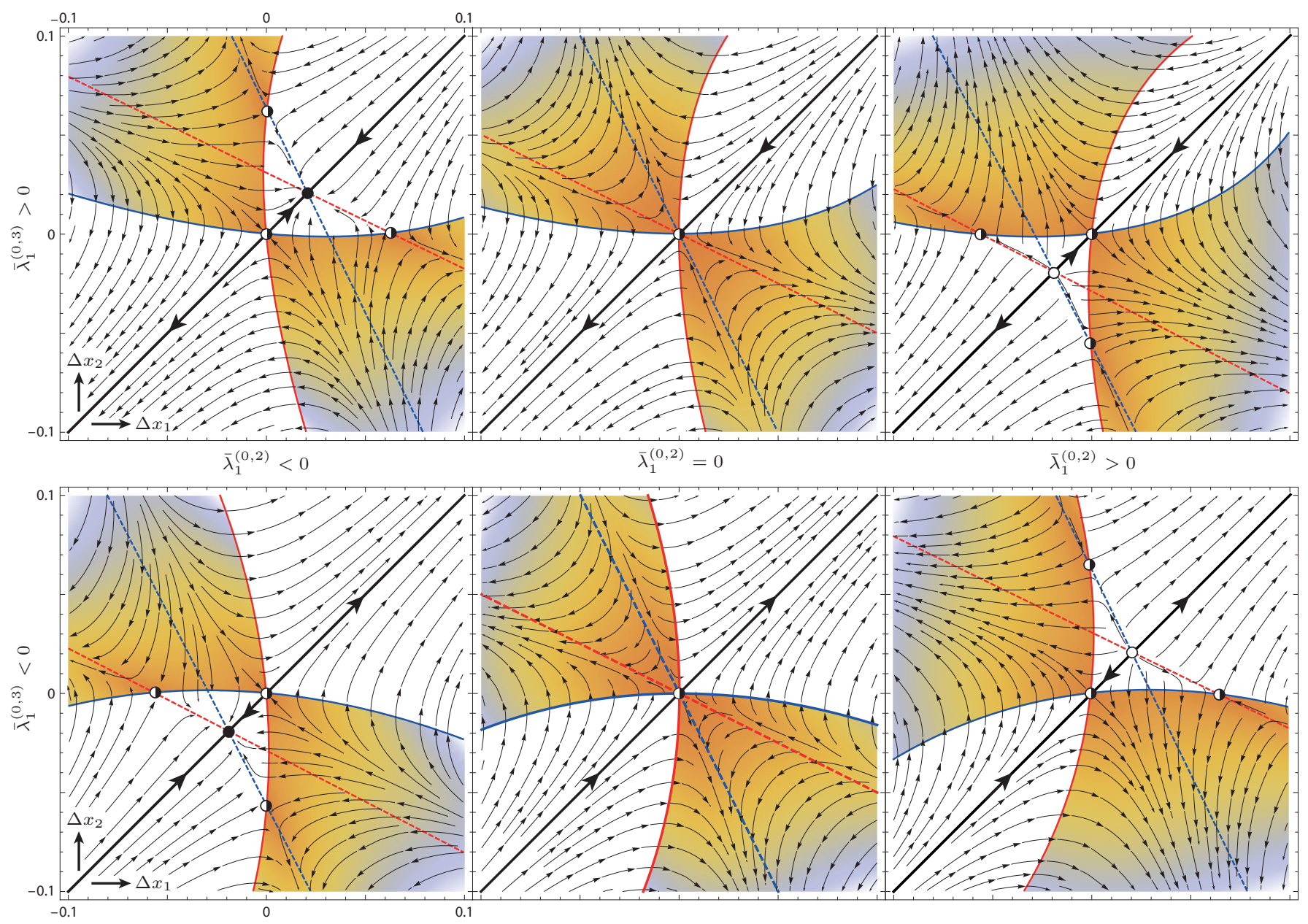

Fig. 4. Unfolding of the branching bifurcation using model $(31,34,35)$. The unfolding parameter $\bar{\lambda}_{1}^{(0,2)}$ increases from left to right and vanishes in the central panel, in which all equilibria E0-E3 collide at $\left(\Delta x_{1}, \Delta x_{2}\right)=(0,0)$. Top/bottom rows: $\bar{\lambda}_{1}^{(0,3)} \gtrless 0$. The resident-mutant coexistence region is shaded, with color code orange-to-blue measuring the magnitude of the vector field. The region boundary $1\left(\eta_{1}\left(\Delta x_{1}, \Delta x_{2}\right)=0(31)\right)$ and the internal $x_{1}$-nullcline $\left(s_{1}\left(\Delta x_{1}, \Delta x_{2}\right)=0(34)\right)$ are plotted in blue (solid and dashed); red for boundary $2\left(\eta_{2}\left(\Delta x_{1}, \Delta x_{2}\right)=0(31)\right)$ and the internal $x_{2}$-nullcline $\left(s_{2}\left(\Delta x_{1}, \Delta x_{2}\right)=0(34)\right)$. Full points: stable equilibria; half-filled points: saddles; empty points: repellor equilibria.

of model $(31,34,35)$ are show in Fig. 4 together with the coexistence region $\tilde{n}_{i}\left(\Delta x_{1}, \Delta x_{2}\right) \geq 0, i=1,2$, defined in $(31,32)$. Note the different curvatures of the coexistence region boundaries in the two cases (top and bottom panels, respectively; the curvature of the locally vertical boundary is given in (19), $\lessgtr 0$ for

$\left.4 \quad \bar{\lambda}_{1}^{(0,3)} \gtrless 0\right)$. Then $\bar{\lambda}_{1}^{(0,3)} \gtrless 0$ makes branching possible at the bifurcation only for mutants with larger/smaller trait values (as already anticipated, without a formal derivation, in [Kisdi, 1999]). In both cases branching 6 is possible, under (G2), so the singular strategy is a branching point at the bifurcation.

\section{Examples}

\section{5.1. Branching in a single species model of asymmetric competition}

We first consider the single species AD model of asymmetric competition described in [Kisdi, 1999]. The 10 resident-mutant model (1) reads:

$$
\begin{aligned}
& \dot{n}_{1}=n_{1}\left(\rho\left(x_{1}\right)-\alpha(0) n_{1}-\alpha\left(x_{1}-x_{2}\right) n_{2}\right), \\
& \dot{n}_{2}=n_{2}\left(\rho\left(x_{2}\right)-\alpha\left(x_{2}-x_{1}\right) n_{1}-\alpha(0) n_{2}\right),
\end{aligned}
$$


with Gaussian $\rho(x)=\exp \left(-x^{2} / 2 \sigma^{2}\right)$ and sigmoidal $\alpha(x)=1-\frac{1}{1+\nu \exp (-x)}, \sigma, \nu>0$, that is built on the $g$-function

$$
g\left(n_{1}, n_{2}, x_{1}, x_{2}, x^{\prime}\right)=\rho\left(x^{\prime}\right)-\alpha\left(x^{\prime}-x_{1}\right) n_{1}-\alpha\left(x^{\prime}-x_{2}\right) n_{2} .
$$

Model (38) is simple enough (Lotka-Volterra competition) that we can solve analytically for all the 4 relevant quantities: the monomorphic and dimorphic resident equilibrium densities

$$
\bar{n}(x)=\rho(x) / \alpha(0), \quad \bar{n}_{1}\left(x_{1}, x_{2}\right)=\frac{\rho\left(x_{1}\right) \alpha(0)-\rho\left(x_{2}\right) \alpha\left(x_{1}-x_{2}\right)}{\alpha(0)^{2}-\alpha\left(x_{1}-x_{2}\right) \alpha\left(x_{2}-x_{1}\right)}, \quad \bar{n}_{2}\left(x_{1}, x_{2}\right)=\bar{n}_{1}\left(x_{2}, x_{1}\right),
$$

the monomorphic and dimorphic fitnesses

$$
\begin{aligned}
\lambda_{1}\left(x, x^{\prime}\right)=g\left(\bar{n}(x), 0, x, x, x^{\prime}\right) & =\rho\left(x^{\prime}\right)-\alpha\left(x^{\prime}-x\right) \bar{n}(x), \\
\lambda_{2}\left(x_{1}, x_{2}, x^{\prime}\right)=g\left(\bar{n}_{1}\left(x_{1}, x_{2}\right), \bar{n}_{2}\left(x_{1}, x_{2}\right), x_{1}, x_{2}, x^{\prime}\right) & =\rho\left(x^{\prime}\right)-\alpha\left(x^{\prime}-x_{1}\right) \bar{n}_{1}\left(x_{1}, x_{2}\right)-\alpha\left(x^{\prime}-x_{2}\right) \bar{n}_{2}\left(x_{1}, x_{2}\right),
\end{aligned}
$$

6 the monomorphic and dimorphic selection gradients

$$
\begin{aligned}
\lambda_{1}^{(0,1)}(x, x) & =\rho^{(1)}(x)-\frac{\alpha^{(1)}(0)}{\alpha(0)} \rho(x)=\frac{\sigma^{2}-\nu x-x}{\sigma^{2}(1+\nu) \exp \left(x^{2} / 2 \sigma^{2}\right)}, \\
\lambda_{2}^{(0,0,1)}\left(x_{1}, x_{2}, x_{i}\right) & =\rho^{(1)}\left(x_{i}\right)-\alpha^{(1)}\left(x_{i}-x_{1}\right) \bar{n}_{1}\left(x_{1}, x_{2}\right)-\alpha^{(1)}\left(x_{i}-x_{2}\right) \bar{n}_{2}\left(x_{1}, x_{2}\right), \quad i=1,2,
\end{aligned}
$$

the singular strategy

$$
\bar{x}=\sigma^{2} /(1+\nu),
$$

8 annihilating $\lambda_{1}^{(0,1)}(x, x)$, the fitness second derivatives ruling branching at $\bar{x}$

$$
\begin{aligned}
\bar{\lambda}_{1}^{(1,1)} & =\frac{\alpha^{(2)}(0)}{\alpha(0)} \rho(\bar{x})-\frac{\alpha^{(1)}(0)}{\alpha(0)} \rho^{(1)}(\bar{x})=-\frac{\nu}{(1+\nu)^{2} \exp \left(\sigma^{2} / 2(1+\nu)^{2}\right)}, \\
\bar{\lambda}_{1}^{(0,2)}=\rho^{(2)}(\bar{x})-\frac{\alpha^{(2)}(0)}{\alpha(0)} \rho(\bar{x}) & =-\frac{(1+\nu)^{2}-\nu \sigma^{2}}{\sigma^{2}(1+\nu)^{2} \exp \left(\sigma^{2} / 2(1+\nu)^{2}\right)},
\end{aligned}
$$

and the third derivatives entering our approximations

$$
\begin{aligned}
\bar{\lambda}_{1}^{(2,1)}=-\frac{\alpha^{(3)}(0)}{\alpha(0)} \rho(\bar{x})+2 \frac{\alpha^{(2)}(0)}{\alpha(0)} \rho^{(1)}(\bar{x})-\frac{\alpha^{(1)}(0)}{\alpha(0)} \rho^{(2)}(\bar{x}) & =\frac{\nu^{2} \sigma^{2}-\nu^{2}-2 \nu \sigma^{2}-2 \nu-1}{\sigma^{2}(1+\nu)^{3} \exp \left(\sigma^{2} / 2(1+\nu)^{2}\right)}, \\
\bar{\lambda}_{1}^{(1,2)}=\frac{\alpha^{(3)}(0)}{\alpha(0)} \rho(\bar{x})-\frac{\alpha^{(2)}(0)}{\alpha(0)} \rho^{(1)}(\bar{x}) & =\frac{\nu(3-\nu)}{(1+\nu)^{3} \exp \left(\sigma^{2} / 2(1+\nu)^{2}\right)} \\
\bar{\lambda}_{1}^{(0,3)}=\rho^{(3)}(\bar{x})-\frac{\alpha^{(3)}(0)}{\alpha(0)} \rho(\bar{x}) & =\frac{\nu^{2} \sigma^{2}+3 \nu^{2}-4 \nu \sigma^{2}+6 \nu+3}{\sigma^{2}(1+\nu)^{3} \exp \left(\sigma^{2} / 2(1+\nu)^{2}\right)} .
\end{aligned}
$$

It is easy to verify that the singular strategy $\bar{x}$ is attracting the monomorphic evolutionary dynamics for any positive $(\sigma, \nu)$ (eigenvalue $\left.\bar{\lambda}_{1}^{(1,1)}+\bar{\lambda}_{1}^{(0,2)}=-\left(\sigma^{2} \exp \left(\sigma^{2} / 2(1+\nu)^{2}\right)\right)^{-1}<0\right)$, that coexistence in its 12 vicinity is always possible $\left(\bar{\lambda}_{1}^{(1,1)}<0\right.$ for $\left.\nu>0\right)$, and that branching $\left(\bar{\lambda}_{1}^{(0,2)}>0\right)$ occurs for $\sigma^{2}>(1+\nu)^{2} / \nu$. At $\sigma^{2}=(1+\nu)^{2} / \nu$ the system undergoes the branching bifurcation. Increasing the value of $\sigma$, we pass 14 from a terminal to a branching point.

Fig. 5 compares our approximated model $(31,34,35)$ and coexistence region boundaries $\eta_{i}\left(\Delta x_{1}, \Delta x_{2}\right)=$ $0, i=1,2(31)$, with the fully nonlinear versions. As in Fig. 4, the boundary 1 of the coexistence region and the internal $x_{1}$-nullcline of the dimorphic evolutionary dynamics are plotted in blue (solid and dashed); red

${ }_{18}$ for boundary 2 and the internal $x_{2}$-nullcline. Lighter colors are used for the fully nonlinear versions. The values of the model parameters are reported in the caption and those of the genericity conditions left-hand sides, $\bar{\lambda}_{1}^{(1,1)}$ (G1) and $\bar{\lambda}_{1}^{(0,3)}$ (G2) can be checked below each figure panel.

\subsection{Prey branching in a prey-predator community}

22 As a second example, we consider the multi-species prey-predator AD model described in [Landi et al., 2013]. Using the notation introduced in Appendix C, the resident-mutant model (1) after a mutation in 

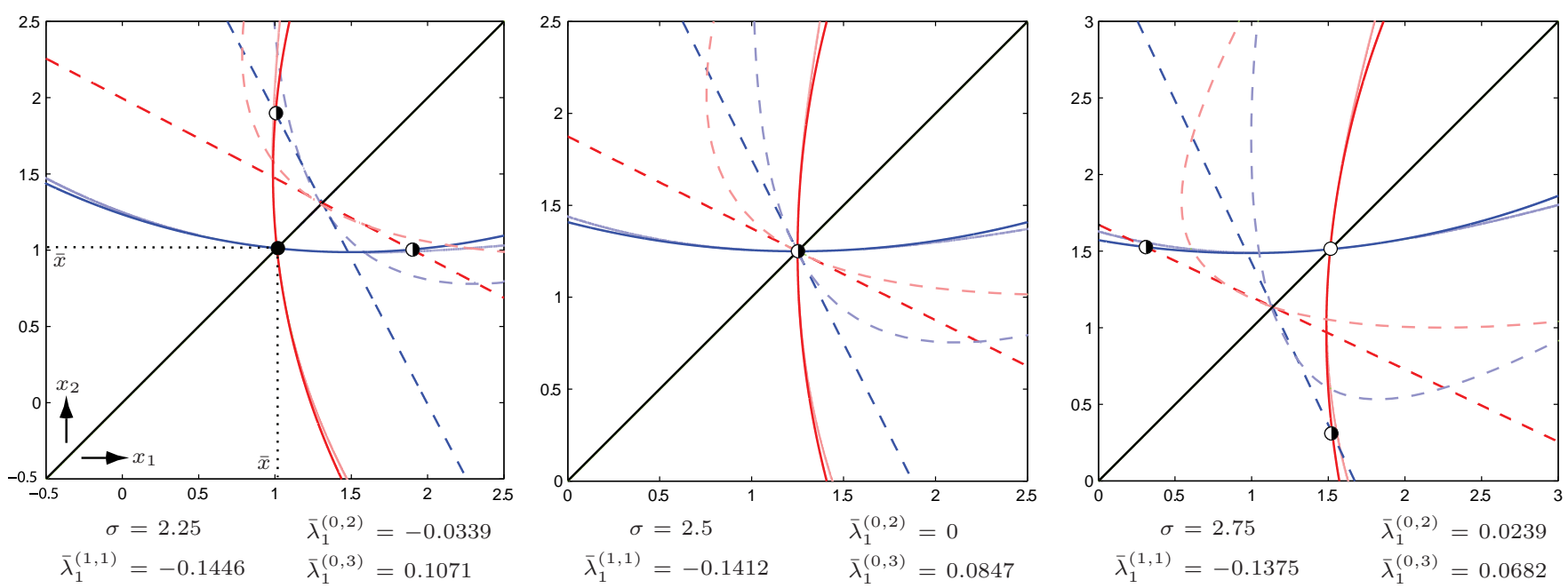

Fig. 5. Unfolding of the branching bifurcation in the AD model in [Kisdi, 1999]. The model parameter $\sigma$ increases from left to right turning the singular strategy $\bar{x}$ from a terminal $\left(\bar{\lambda}_{1}^{(0,2)}<0\right)$ to a branching $\left(\bar{\lambda}_{1}^{(0,2)}>0\right.$ ) point (other parameter: $\left.\nu=4\right)$. The approximations $\eta_{i}\left(\Delta x_{1}, \Delta x_{2}\right)=0$ (31) and $s_{i}\left(\Delta x_{1}, \Delta x_{2}\right)=0$ (34) of the coexistence region boundaries and of the internal $x_{i}$-nullcline, $i=1,2$, are shown around $(\bar{x}, \bar{x})$ using the same graphical and color codes of Fig. 4. Lighter colors are used for the fully nonlinear versions: boundary $1, \lambda_{1}\left(x_{2}, x_{1}\right)=0$; boundary $2, \lambda_{1}\left(x_{1}, x_{2}\right)=0$; and $x_{i}$-nullcline, $\lambda_{2}^{(0,0,1)}\left(x_{1}, x_{2}, x_{i}\right)=0$.

$$
\begin{aligned}
& \dot{n}_{1}=n_{1}\left(r-c\left(x_{1}, x_{1}\right) n_{1}-c\left(x_{1}, x_{2}\right) n_{2}-\frac{a\left(x_{1}, X\right)}{1+a\left(x_{1}, X\right) h\left(x_{1}, X\right) n_{1}+a\left(x_{2}, X\right) h\left(x_{2}, X\right) n_{2}} N\right), \\
& \dot{n}_{2}=n_{2}\left(r-c\left(x_{2}, x_{1}\right) n_{1}-c\left(x_{2}, x_{2}\right) n_{2}-\frac{a\left(x_{2}, X\right)}{1+a\left(x_{1}, X\right) h\left(x_{1}, X\right) n_{1}+a\left(x_{2}, X\right) h\left(x_{2}, X\right) n_{2}} N\right), \\
& \dot{N}=N\left(e \frac{a\left(x_{1}, X\right) n_{1}+a\left(x_{2}, X\right) n_{2}}{1+a\left(x_{1}, X\right) h\left(x_{1}, X\right) n_{1}+a\left(x_{2}, X\right) h\left(x_{2}, X\right) n_{2}}-d\right),
\end{aligned}
$$

with valley-shaped prey intra-specific competition

$$
c\left(x_{1}, x_{2}\right)=\frac{\gamma_{1}+\gamma_{2} x_{1}^{2}}{1+\gamma_{0}\left(\gamma_{1}+\gamma_{2} x_{1}^{2}\right)} \exp \left(-\frac{1}{4}\left(x_{1}-x_{2}\right)^{2}\right),
$$

bell-shaped predator attack rate

$$
a(x, X)=\alpha_{0}+\exp \left(-(x-X)^{2}\right),
$$

and sigmoidal predator handling time

$$
h(x, X)=\theta\left(\frac{3}{2}-\frac{1}{1+\exp \left(\theta_{3} x\right)}\right)\left(\frac{3}{2}-\frac{1}{1+\exp \left(-\theta_{4} X\right)}\right) .
$$

It is built on the $g$-function

$$
g\left(n_{1}, n_{2}, N, x_{1}, x_{2}, X, x^{\prime}\right)=r-c\left(x^{\prime}, x_{1}\right) n_{1}-c\left(x^{\prime}, x_{2}\right) n_{2}-\frac{a\left(x^{\prime}, X\right)}{1+a\left(x_{1}, X\right) h\left(x_{1}, X\right) n_{1}+a\left(x_{2}, X\right) h\left(x_{2}, X\right) n_{2}} N .
$$

Analytically, we can only compute the monomorphic resident equilibrium

$$
\bar{n}(x, X)=\frac{d}{a(x, X)(e-d h(x, X))}, \quad \bar{N}(x, X)=\left(\frac{r-c(x, x) \bar{n}(x, X)}{a(x, X)}\right)(1+a(x, X) h(x, X) \bar{n}(x, X)),
$$

the prey monomorphic fitness

$$
\begin{array}{r}
\lambda_{1}\left(x, X, x^{\prime}\right)=g\left(\bar{n}(x, X), 0, \bar{N}(x, X), x, x, X, x^{\prime}\right)=r-c\left(x^{\prime}, x\right) \bar{n}(x, X) \\
-\frac{a\left(x^{\prime}, X\right)}{1+a(x, X) h(x, X) \bar{n}(x, X)} \bar{N}(x, X),
\end{array}
$$


and selection gradient

$$
\lambda_{1}^{(0,0,1)}(x, X, x)=-c^{(1,0)}(x, x) \bar{n}(x, X)-\frac{a^{(1,0)}(x, X)}{1+a(x, X) h(x, X) \bar{n}(x, X)} \bar{N}(x, X),
$$

as well as the fitness second and third derivatives $\left(\lambda_{1}^{(1,0,1)}\right.$ and $\lambda_{1}^{(0,0,2)}$ ruling prey branching, and $\lambda_{1}^{(2,0,1)}$, $\lambda_{1}^{(1,0,2)}$, and $\lambda_{1}^{(0,0,3)}$ entering our approximations).

$4 \quad$ All other relevant quantities must be computed numerically:

- the singular coalition $(\bar{x}, \bar{X})$, by simulating the coevolution of both prey and predator (see [Landi et al., 2013] for the modeling of predator mutations),

- the prey singular strategy $\bar{x}(X)$ at given predator trait, by solving $\lambda_{1}^{(0,0,1)}(x, X, x)=0$ for $x$,

- the fitness second and third derivatives $\bar{\lambda}_{1}^{(1,0,1)}, \bar{\lambda}_{1}^{(0,0,2)}, \bar{\lambda}_{1}^{(2,0,1)}, \bar{\lambda}_{1}^{(1,0,2)}$, and $\bar{\lambda}_{1}^{(0,0,3)}$, simply evaluating the corresponding analytical expressions at $(\bar{x}, \bar{X})$,

- the boundaries 1 and 2 of the resident-mutant coexistence region rooted at $(\bar{x}(X), \bar{x}(X))$ in the plane $\left(x_{1}, x_{2}\right)$, by continuing the contour-lines $\lambda_{1}\left(x_{2}, X, x_{1}\right)=0$ and $\lambda_{1}\left(x_{1}, X, x_{2}\right)=0$, respectively,

- the dimorphic resident equilibrium densities $\bar{n}_{1}\left(x_{1}, x_{2}, X\right), \bar{n}_{2}\left(x_{1}, x_{2}, X\right)$, and $\bar{N}\left(x_{1}, x_{2}, X\right)$, by continuing the nontrivial equilibrium solution of model (39) w.r.t. $\left(x_{1}, x_{2}\right)$ treated as model parameters,

- the $x_{i}$-nullcline of the dimorphic coevolutionary dynamics, by continuing the contour-line

$$
\begin{aligned}
\lambda_{2}^{(0,0,0,1)}\left(x_{1}, x_{2}, X, x_{i}\right)=-c^{(1,0)}\left(x_{i}, x_{1}\right) \bar{n}_{1}\left(x_{1}, x_{2}, X\right)-c^{(1,0)}\left(x_{i}, x_{2}\right) \bar{n}_{2}\left(x_{1}, x_{2}, X\right) & \\
- & \frac{a^{(1,0)}\left(x_{i}, X\right)}{1+a\left(x_{1}, X\right) h\left(x_{1}, X\right) \bar{n}_{1}\left(x_{1}, x_{2}, X\right)+a\left(x_{2}, X\right) h\left(x_{2}, X\right) \bar{n}_{2}\left(x_{1}, x_{2}, X\right)} \bar{N}\left(x_{1}, x_{2}, X\right)=0
\end{aligned}
$$

$i=1,2$, together with the equilibrium densities $\bar{n}_{1}\left(x_{1}, x_{2}, X\right), \bar{n}_{2}\left(x_{1}, x_{2}, X\right)$, and $\bar{N}\left(x_{1}, x_{2}, X\right)$.

From the analysis in [Landi et al., 2013], we know that an attracting singular coalition $(\bar{x}, \bar{X})$ exists for broad ranges of the model parameters and a prey-branching bifurcation occurs by increasing the predator efficiency $e$ (see Eq.(39c)). Fixing the model parameters to the values reported in the caption and always keeping $X=\bar{X}$, Fig. 6 compares our approximated model $(31,34,35)$ and coexistence region boundaries $\eta_{i}\left(\Delta x_{1}, \Delta x_{2}\right)=0, i=1,2(31)$, with the fully nonlinear versions. As in Fig. 4 , the boundary 1 of the

20 coexistence region and the internal $x_{1}$-nullcline of the dimorphic coevolutionary dynamics are plotted in blue (solid and dashed); red for boundary 2 and the internal $x_{2}$-nullcline. Lighter colors are used for the 22 fully nonlinear versions. The genericity of the bifurcation is granted by the values of $\bar{\lambda}_{1}^{(1,0,1)}$ (C.G1) and $\bar{\lambda}_{1}^{(0,0,3)}$ (C.G2) reported below each figure panel.

$24 \quad$ Fig. 7 shows three cases of dimorphic coevolutionary dynamics at incipient branching. In case (a), the prey traits are initialized along the anti-diagonal of the coexistence region, i.e.,

$$
x_{1}(0)=\bar{x}(X(0))+\varepsilon \cos \theta, \quad x_{2}(0)=\bar{x}(X(0))+\varepsilon \sin \theta, \quad \theta=\frac{3}{4} \pi,
$$

${ }_{26}$ and the predator trait $X$ is initially set at its singular value $\bar{X}$. As predicted by our analysis in Appendix $\mathrm{C}$ (by the leading terms of the dimorphic model (C.11) and by the scaled approximations (C.12) of the

${ }_{28}$ coexistence equilibrium densities $\bar{n}_{1}\left(x_{1}, x_{2}, X\right)$ and $\bar{n}_{2}\left(x_{1}, x_{2}, X\right)$ ), the prey traits $x_{1}$ and $x_{2}$ initially diverge symmetrically w.r.t. the singular value $\bar{x}$ (dashed), while the population remains split into two halves (same

so gray scale, see caption) and the predator trait is under neutral selection ( $\varepsilon$ being small but finite, a weak selection acts on $X$ in the very initial phase of branching, showed in the left stretched panel).

In case (b), the prey traits are initialized close to the boundary 2 of the coexistence region (as in (40) with $\left.\theta=\frac{1}{2} \pi\right)$ and the predator trait still at the singular value $\bar{X}$. Close to boundary 2 the prey population is 34 almost monomorphic and mainly composed of $x_{1}$-individuals (see the gray scale in the left panel, basically black in $x_{1}$ and white in $\left.x_{2}\right)$, so $x_{1}(0)$ and $X(0)$ are almost at equilibrium at the singular coalition $(\bar{x}, \bar{X})$. In

${ }_{36}$ accordance with our analysis of Sects. 3 and 4 and of Appendix C, the branching dynamics of $\left(x_{1}, x_{2}\right)$ point toward the anti-diagonal and this, at the same time, equilibrate the equilibrium densities $\bar{n}_{1}\left(x_{1}, x_{2}, X\right)$ and ${ }_{38} \bar{n}_{2}\left(x_{1}, x_{2}, X\right)$ (see the gray scale in the right panel). Note that in the very initial phase of branching (left panel), while $x_{1}$ decreases following the branching dynamics, evolution is still basically monomorphic and 

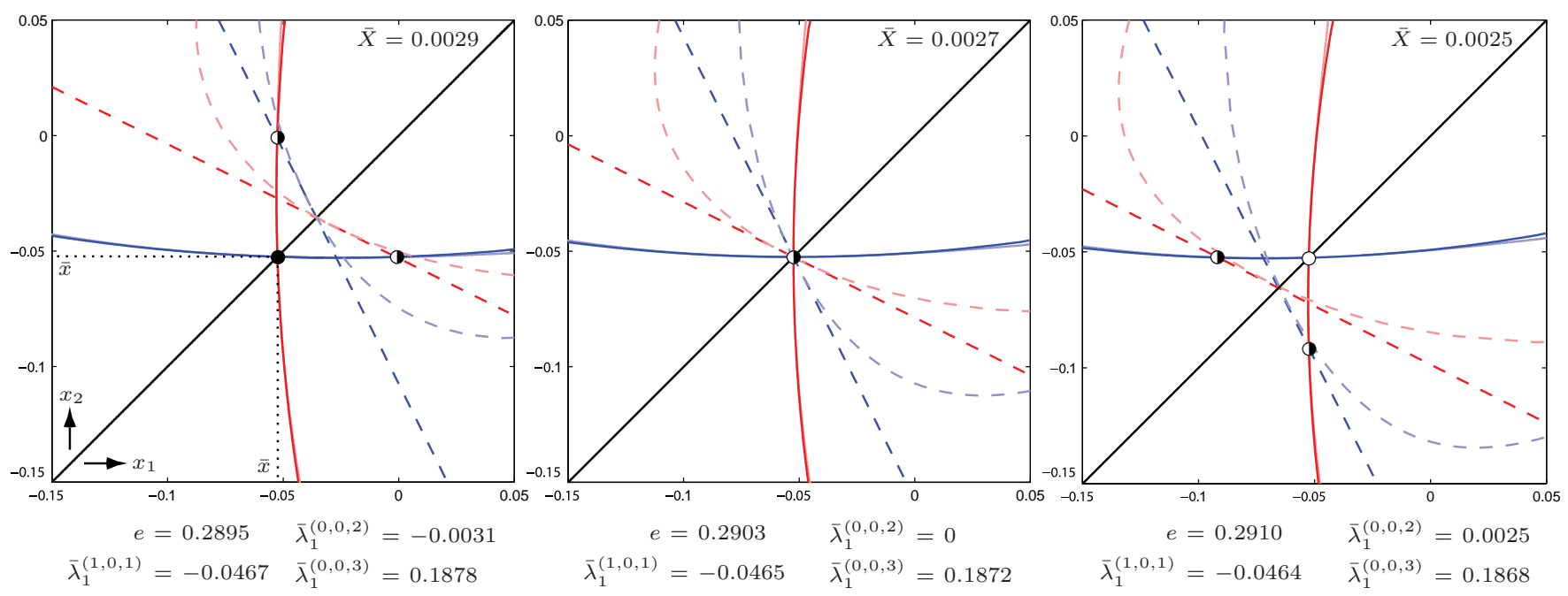

Fig. 6. Unfolding of the branching bifurcation in the AD model in [Landi et al., 2013]. The model parameter $e$ increases from left to right turning the singular coalition $(\bar{x}, \bar{X})$ from a terminal $\left(\bar{\lambda}_{1}^{(0,0,2)}<0\right)$ to a branching $\left(\bar{\lambda}_{1}^{(0,0,2)}>0\right)$ point (other parameters: $r=0.5, d=0.05, \gamma_{0}=0.01, \gamma_{1}=0.5, \gamma_{2}=2.3, \alpha_{0}=0.01, \theta=0.5, \theta_{3}=\theta_{4}=5$ ). The approximations $\eta_{i}\left(\Delta x_{1}, \Delta x_{2}\right)=0$ (31) and $s_{i}\left(\Delta x_{1}, \Delta x_{2}\right)=0$ (34) of the coexistence region boundaries and of the internal $x_{i}$-nullcline, $i=1,2$, are shown around $(\bar{x}, \bar{x})$ in the $\left(x_{1}, x_{2}\right)$ plane, using the same graphical and color codes of Fig. 4. Lighter colors are used for the fully nonlinear versions: boundary $1, \lambda_{1}\left(x_{2}, \bar{X}, x_{1}\right)=0$; boundary $2, \lambda_{1}\left(x_{1}, \bar{X}, x_{2}\right)=0$; and $x_{i}$-nullcline, $\lambda_{2}^{(0,0,0,1)}\left(x_{1}, x_{2}, \bar{X}, x_{i}\right)=0, i=1,2$. Numerical continuation performed with the software package Matcont [Dhooge et al. $2002]$.

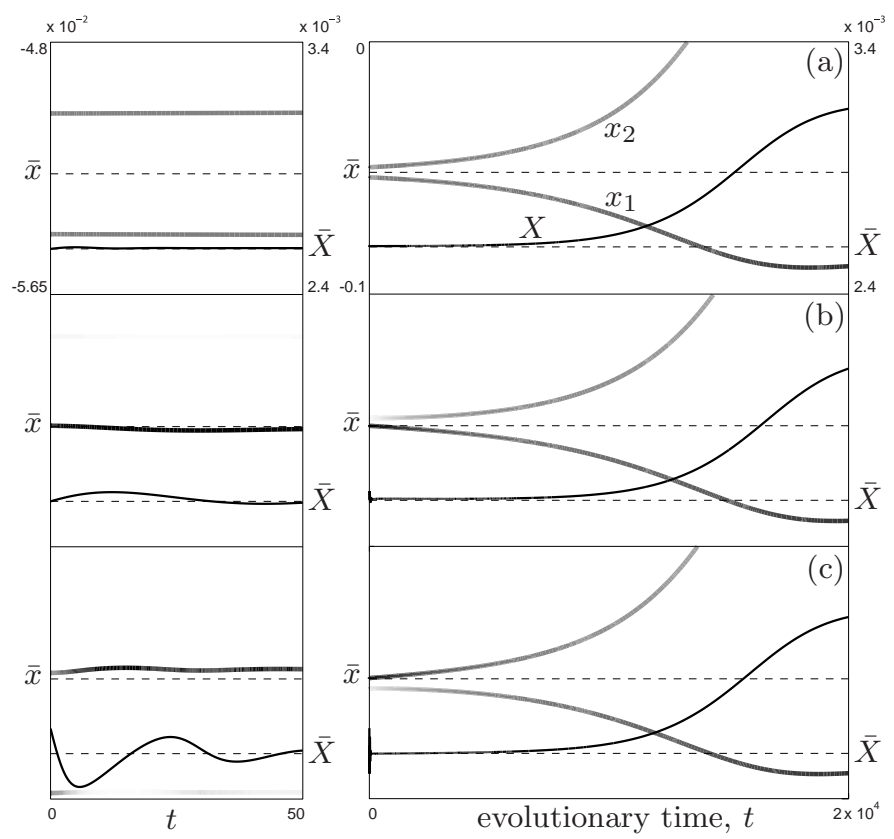

Fig. 7. Three examples of dimorphic coevolutionary dynamics at incipient branching in the AD model in [Landi et al., 2013]. Case (a): $x_{1}(0)=\bar{x}-\frac{\sqrt{2}}{2} \varepsilon, x_{2}(0)=\bar{x}+\frac{\sqrt{2}}{2} \varepsilon, X(0)=\bar{X}$. Case $(\mathrm{b}): x_{1}(0)=\bar{x}, x_{2}(0)=\bar{x}+\varepsilon, X(0)=\bar{X}$. Case (c): $x_{1}(0)=\bar{x}(\bar{X}+\delta)-\frac{\sqrt{2}}{2} \varepsilon, x_{2}(0)=\bar{x}(\bar{X}+\delta)+\frac{\sqrt{2}}{2} \varepsilon, X(0)=\bar{X}+\delta$. Parameter values as in Fig. 6 (right), $\varepsilon=0.003, \delta=0.0001$. The gray scale in the $x_{i}$-time-series indicates the relative density $\bar{n}_{i}\left(x_{1}, x_{2}, X\right) /\left(\bar{n}_{1}\left(x_{1}, x_{2}, X\right)+\bar{n}_{2}\left(x_{1}, x_{2}, X\right)\right), i=1,2$.

$\left(x_{1}, X\right)$ oscillate around $(\bar{x}, \bar{X})$, being the singular coalition a stable focus $\left(x_{1}\right.$ - and $X$-oscillations have similar amplitude, though this is not visible at the scale used for $\left.\left(x_{1}, x_{2}\right)\right)$. As the density of $x_{2}$ grows in detriment of that of $x_{1}$, the branching dynamics take over as in case (a). However, while $x_{1}$-individuals are 
still predominant, the selection pressure on the predator is lower (if not slightly negative, due to adaptation 2 to the decreasing trait $x_{1}$ ) w.r.t. case (a) and, as a result, the eventual increase of $X$ is delayed.

In case (c), the prey traits are initialized along the anti-diagonal (as in case (a), according to (40)), 4 but the predator trait is perturbed from singularity, $X(0)=\bar{X}+\delta$. As a result, being $\bar{x}>\bar{x}(\bar{X}+\delta)$, we have $x_{1}(0)<\bar{x}$ and $x_{2}(0)$ slightly above $\bar{x}$, so point $\left(x_{1}(0), x_{2}(0)\right)$ is internal (and close to boundary 1 )

6 to the prey coexistence region rooted at $(\bar{x}, \bar{x})$ in the $\left(x_{1}, x_{2}\right)$ plane. The predator trait $X$ is initially out of equilibrium and quickly evolves toward $\bar{X}$ (left panel). The oscillations of the predator trait induce a 8 corresponding movement (back and forth) of the prey coexistence region, that puts point $\left(x_{1}, x_{2}\right)$ close to boundary 1 , avoiding however missing the branching $\left(\left(x_{1}, x_{2}\right)\right.$ touching the region boundary). Thus, after 10 a first quick transient (see the gray scale in the left panel, soon becoming lighter in $x_{1}$ and darker in $x_{2}$ ), the situation is symmetric w.r.t. case (b), with the prey population mainly composed of $x_{2}$-individuals.

\section{$12 \quad 6$. Discussion and conclusions}

The main theoretical contribution of this paper is a general method of approximating the dimorphic

14 fitness (8). It is based on a radial expansion (w.r.t. $\varepsilon$ ) on a given ray (identified by the angle $\theta$ ) in the plane $\left(x_{1}, x_{2}\right)$ of the two similar coexisting strategies. It exploits the fact (observed in [Durinx, 2008] and [Dercole

16 \& Geritz, 2015]) that the equilibrium densities $\bar{n}_{1}\left(x_{1}, x_{2}\right)$ and $\bar{n}_{2}\left(x_{1}, x_{2}\right)$, at which the two strategies can coexist (under (G1)) for $x_{1}$ and $x_{2}$ close to the singular strategy $\bar{x}$, are well defined and smooth along each

18 given ray in the cone of coexistence rooted at $(\bar{x}, \bar{x})$, though nonsmooth at $(\bar{x}, \bar{x})$. As a consequence, the $\varepsilon$-expansions of the densities $\bar{n}_{1}(\varepsilon, \theta)$ and $\bar{n}_{2}(\varepsilon, \theta)$ and of the dimorphic fitness $\lambda_{2}\left(\varepsilon, \theta, \Delta x^{\prime}\right)$ (redefined in

20 polar coordinates $(\varepsilon, \theta)$ with $\left.\Delta x^{\prime}:=x^{\prime}-\bar{x}\right)$ are $\theta$-dependent but, interestingly, they can be written back in terms of rational $\left(\bar{n}_{1}\right.$ and $\left.\bar{n}_{2}\right)$ and polynomial $\left(\lambda_{2}\right)$ expressions of $\left(x_{1}, x_{2}\right)$. The resulting expressions are not

22 expansions w.r.t. $\left(x_{1}, x_{2}\right)$ - such expansions cannot be defined, contrary to what originally done in [Geritz et al., 1997, 1998] - but can nevertheless be used as approximations in the resident-mutant coexistence ${ }_{24}$ region locally to the singular point $(\bar{x}, \bar{x})$.

Our methodology is quite general. Other non-similar resident populations (of the same or different 26 species) are considered (in Appendix $\mathrm{C}$ ) and the approximation can be taken up to any order (in $\varepsilon$ ). Thanks to a structural property assumed for the dimorphic fitness (property P4 in Sect.2.1, recently introduced in [Dercole, 2014]), the $\bar{n}_{1}, \bar{n}_{2}$, and $\lambda_{2} \varepsilon$-expansions can be written in terms of the geometry of the monomorphic fitness (3) (in contrast to what preliminarily found in [Durinx, 2008] in the special case of Lotka-Volterra models).

We have used the developed approach to unfold the branching bifurcation, at which a stable equilibrium of the monomorphic AD canonical equation (4) loses evolutionary stability. Specifically, assuming (G1) - allowing resident-mutant coexistence - and (G2) - ensuring the transversality and genericity of the

${ }_{34}$ bifurcation - we have unfolded the transition of $\bar{\lambda}_{1}^{(0,2)}$ across zero - the evolutionary equilibrium turning from a terminal $\left(\bar{\lambda}_{1}^{(0,2)}<0\right)$ to a branching $\left(\bar{\lambda}_{1}^{(0,2)}>0\right)$ point of AD.

36 At the bifurcation, the evolutionary dynamics ruled by the dimorphic canonical equation (7) are dominated by the third-order terms in the $\varepsilon$-expansion of the dimorphic fitness (8). Interestingly, the 3 second-order terms coincide with those Geritz et al. [1997, 1998] obtained by assuming smoothness, though nongeneric constraints on the monomorphic fitness come along at second- as well as at higher-orders (see

40 Appendix 1). Thus the (second-order) branching condition (9) of [Geritz et al., 1997, 1998] is correct and our approach becomes essential only at third-order.

42 By means of a smooth coordinate change and time-scaling, we have identified a simple model that is locally equivalent to the dimorphic canonical equation (7) and shows the bifurcation. We claim this is the 44 normal form for the branching bifurcation: model $(34,35,37)$ restricted to the resident-mutant coexistence region $\tilde{n}_{i}\left(\Delta x_{1}, \Delta x_{2}\right) \geq 0, \Delta x_{i}:=x_{i}-\bar{x}, i=1,2$, defined in $(36,37)$, locally to $\left(\Delta x_{1}, \Delta x_{2}\right)=(0,0)$. The

46 model depends on four parameters that are all monomorphic fitness derivatives: the unfolding parameter $\bar{\lambda}_{1}^{(0,2)}$, the fitness cross-derivative $\bar{\lambda}_{1}^{(1,1)}$ (constrained by $(\mathrm{G} 1)$ ), the normal form coefficient $\bar{\lambda}_{1}^{(0,3)}$ (constrained 48 by $(\mathrm{G} 2)$ ), and $\bar{\lambda}_{1}^{(1,2)}$ that plays no role and could be eliminated by a further coordinate change (in that sense the normal form could be simplified, but this would require a further change of coordinates that we 


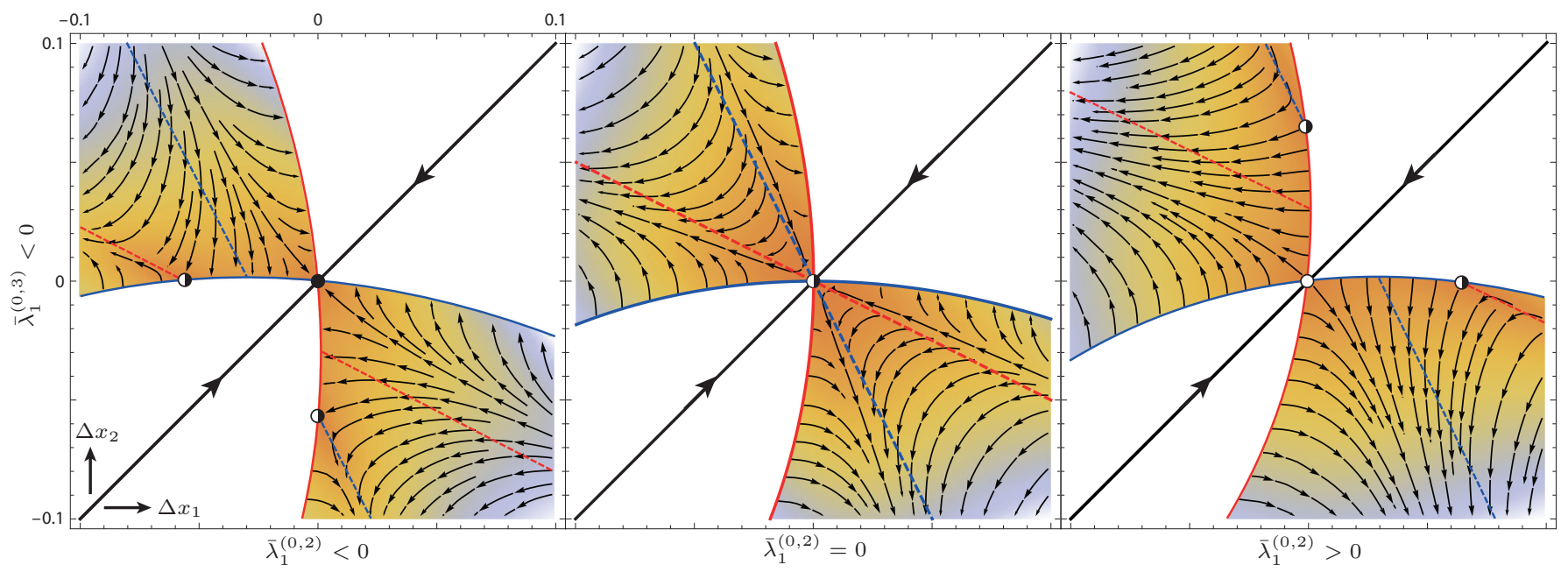

Fig. 8. Monomorphic and dimorphic evolutionary dynamics around the branching bifurcation restricted to the region of resident-mutant coexistence (shaded area; the color code orange-to-blue measures the magnitude of the vector field). The singular strategy (the dot at $\left.\left(\Delta x_{1}, \Delta x_{2}\right)=(0,0)\right)$ is always convergence stable (the monomorphic dynamics is shown along the diagonal), whereas it is evolutionarily stable in the left panel (terminal point, full dot) and evolutionary unstable in the central and the right panels (branching bifurcation, half-filled dot; branching point, empty dot). Other graphical and color codes as in Fig. 4.

avoided). The only genericity (and transversality) condition required by the bifurcation (other than the stability of the monomorphic resident equilibrium (10) and the resident-mutant coexistence under (G1)) is then $(\mathrm{G} 2)$.

Keeping into account the curvature of the boundaries of the resident-mutant coexistence region, we have proposed a second model (though introduced as first in Sect. 3 and necessarily less simple), locally equivalent to the dimorphic canonical equation at the incipient branching: model $(31,34,35)$ restricted to the coexistence region $\tilde{n}_{i}\left(\Delta x_{1}, \Delta x_{2}\right) \geq 0, i=1,2$, defined in $(31,32)$. The quadratic approximation of the coexistence region boundaries also depends on $\bar{\lambda}_{1}^{(2,1)}$, which is the last independent third-derivative of the monomorphic fitness to be involved $\left(\bar{\lambda}_{1}^{(3,0)}\right.$ is related to the others by Eq. (13c)). The new approxi10 mation of the boundaries has the advantage of showing some geometric features relating the trajectories of the dimorphic canonical equation with the boundaries themselves. Specifically, the internal $x_{1}$-nullcline ${ }_{12}\left(s_{1}\left(\Delta x_{1}, \Delta x_{2}\right)=0\right.$; dashed blue in Fig. 8) connects to an horizontal fold of boundary $1\left(\tilde{n}_{1}\left(\Delta x_{1}, \Delta x_{2}\right)=0\right.$, solid blue) - both smaller and larger $x_{1}$-mutants do (Fig. 8 left) or do not (Fig. 8 right) invade the monomor14 phic $x_{2}$-population at the fold - and to boundary $2\left(\tilde{n}_{2}\left(\Delta x_{1}, \Delta x_{2}\right)=0\right.$, solid red) at a boundary saddle with $x_{1}=\bar{x}$ - the monomorphic $x_{1}$-population is at equilibrium at the singular strategy; symmetrically for the internal $x_{2}$-nullcline (see [Geritz et al., 1999], the Appendix in particular, for more details).

The analysis of our simplified models as $\bar{\lambda}_{1}^{(0,2)}$ moves across zero unravels the dynamical phenomena 18 turning a terminal point of AD into a branching point. Restricting the model dynamics into the region of $\left(x_{1}, x_{2}\right)$-coexistence, we see (Fig. 8) that the singular point $\left(\Delta x_{1}, \Delta x_{2}\right)=(0,0)$ is always a "corner" equilibrium that is attracting nearby trajectories for $\bar{\lambda}_{1}^{(0,2)}<0$ and repelling for $\bar{\lambda}_{1}^{(0,2)}>0$. The basin of attraction for $\bar{\lambda}_{1}^{(0,2)}<0$ is delimited by the stable manifold of the two boundary saddles (one above and one 22 below the diagonal), the convergence being composed of a dimorphic phase up to the extinction of one of the two similar strategies, followed by a monomorphic phase toward the singular strategy. As $\bar{\lambda}_{1}^{(0,2)}$ moves 24 across zero, the two saddles move along the coexistence region boundaries and cross the diagonal colliding with the singular point, that consequently changes stability (see Sect.4). For $\bar{\lambda}_{1}^{(0,2)}>0$ the trajectories 26 originating close to $(0,0)$ go away from the singularity and eventually reach an evolutionary attractor that is not local to the singularity (and not involved in the bifurcation). The same attractor is generically viable even for $\bar{\lambda}_{1}^{(0,2)}<0$ (see e.g. [Geritz et al., 1999]), but it cannot be reached from a neighborhood of the singularity, unless the mutational step is large enough to escape the basin of attraction. The branching 
bifurcation is therefore catastrophic, in the sense that a small change in $\bar{\lambda}_{1}^{(0,2)}$ triggers a large evolutionary transient leading to a new attractor.

Finally, looking at the curvature of the coexistence region boundaries at the bifurcation $\left(\bar{\lambda}_{1}^{(0,2)}=0\right)$, 4 we can extend (under (G2)) the branching condition (9) to

$$
\lambda_{1}^{(0,2)}(\bar{x}, \bar{x}) \geq 0 .
$$

The natural follow-up to this work is the analysis of the other codimension-one branching bifurcation6 the one at which the fitness cross-derivative $\bar{\lambda}_{1}^{(1,1)}$ vanishes with positive $\bar{\lambda}_{1}^{(0,2)}$. The resident-mutant coexistence region is locally a cusp rooted at the singular point $(\bar{x}, \bar{x})$ (see [Priklopil, 2012, Dercole \& Geritz,

$\therefore$ 2015]), and though there might generically be up to two coexistence equilibria, only one is stable and should be considered for developing a proper expansion of the dimorphic fitness. Further research could

${ }_{10}$ investigate the codimension-two bifurcation at which both fitness second-derivatives vanish (the type of coexistence is already available in [Dercole \& Geritz, 2015]), or the cases at which $\bar{\lambda}_{1}^{(0,3)}=0$ together 12 with one of the fitness second-derivatives; or, as well, higher codimensions that do occur in applications (see, e.g., [Doebeli \& Ispolatov, 2010]). The methodology developed in this paper is readily applicable and 14 convenient to pursue the above projects.

More effort is definitely needed to extend the methodology to the cases of structured populations

${ }_{16}$ [Durinx et al., 2008] and/or to multi-dimensional strategies [Vukics et al., 2003, Ito \& Dieckmann, 2014]. Analogous results are intuitively expected.

\section{Acknowledgements}

This work was supported by the Italian Ministry for University and Research (under contract FIRB RBFR08TIA4).

\section{Appendix A The nonsmoothness of the dimorphic fitness}

22 In this appendix we show that assuming the dimorphic fitness $\lambda_{2}\left(x_{1}, x_{2}, x^{\prime}\right)$ smooth at $(\bar{x}, \bar{x}, \bar{x})$ brings to the nongeneric constraint

$$
\bar{\lambda}_{1}^{(2,0)}=\bar{\lambda}_{1}^{(0,2)}
$$

24 between the second derivatives of the monomorphic fitness $\lambda_{1}\left(x, x^{\prime}\right)$ at $(\bar{x}, \bar{x})$. More in general, in the Supplementary Material (last section) we show that the smoothness assumption implies nongeneric constraints

26 at any order $k \geq 2$ of the form

e.g.

$$
\left.\frac{\partial^{k-1}}{\partial x^{k-1}} \lambda_{1}^{(0,1)}(x, x)\right|_{x=\bar{x}}=0
$$

${ }_{28}$ which is nothing but (A.1) by the neutrality condition (13b), and

$$
\bar{\lambda}_{1}^{(1,1)}+\bar{\lambda}_{1}^{(0,2)}=0 \quad \text { for } \quad k=2
$$

$$
\begin{aligned}
\bar{\lambda}_{1}^{(2,1)}+2 \bar{\lambda}_{1}^{(1,2)}+\bar{\lambda}_{1}^{(0,3)}=0 & \text { for } \quad k=3 \\
\bar{\lambda}_{1}^{(3,1)}+3 \bar{\lambda}_{1}^{(2,2)}+3 \bar{\lambda}_{1}^{(1,3)}+\bar{\lambda}_{1}^{(0,4)}=0 & \text { for } \quad k=4 .
\end{aligned}
$$

The smoothness of the dimorphic fitness would therefore imply that the selection gradient $\lambda_{1}^{(0,1)}(x, x)$ 30 vanishes at $x=\bar{x}$ together with all its $x$-derivatives, whereas all such derivatives are generically expected to be nonzero (see e.g. Eq. (14)), though some of them might vanish in specific models due to symmetries 32 in the trait dependencies.

To show Eq. (A.1) we exploit the consistency properties C1-3 of Sect.2.1 and, in particular, their 34 derivatives w.r.t. $\left(x, x^{\prime}\right)(\mathrm{C} 1),\left(x_{1}, x_{2}, x^{\prime}\right)(\mathrm{C} 2)$, and $\left(x_{1}, x_{2}\right)(\mathrm{C} 3)$ at $x=x_{1}=x_{2}=x^{\prime}=\bar{x}$, that can be taken under smoothness. Specifically, $\mathrm{C} 1$ and its second $x$-derivative give 
C1a: $\bar{\lambda}_{2}^{(0,0,2)}=\bar{\lambda}_{1}^{(0,2)}$,

${ }_{2} \mathrm{C} 1 \mathrm{~b}: \bar{\lambda}_{2}^{(2,0,0)}+2 \bar{\lambda}_{2}^{(1,1,0)}+\bar{\lambda}_{2}^{(0,2,0)}=\bar{\lambda}_{1}^{(2,0)}$,

the second $x_{1}$-derivative of $\mathrm{C} 2$ gives

${ }_{4} \mathrm{C} 2 \mathrm{a}: \bar{\lambda}_{2}^{(2,0,0)}=\bar{\lambda}_{2}^{(0,2,0)}$,

and, in $\mathrm{C} 3$, the mixed $\left(x_{1}, x_{2}\right)$ - and the second $x_{2}$-derivatives of $\lambda_{2}\left(x_{1}, x_{2}, x_{1}\right)=0$ give

${ }_{6} \mathrm{C} 3 \mathrm{a}: \bar{\lambda}_{2}^{(1,1,0)}+\bar{\lambda}_{2}^{(0,1,1)}=0$,

C3b: $\bar{\lambda}_{2}^{(0,2,0)}=0$,

8 whereas the second $x_{2}$-derivative of $\lambda_{2}\left(x_{1}, x_{2}, x_{2}\right)=0$ gives

C3c: $\bar{\lambda}_{2}^{(0,2,0)}+2 \bar{\lambda}_{2}^{(0,1,1)}+\bar{\lambda}_{2}^{(0,0,2)}=0$.

${ }_{10}$ From C1b-C2a-C3b, we therefore conclude

$$
\bar{\lambda}_{2}^{(1,1,0)}=\frac{1}{2} \bar{\lambda}_{1}^{(2,0)}
$$

whereas substituting $\bar{\lambda}_{2}^{(0,1,1)}$ from C3c into C3a and then applying C1a-C3b, we conclude

$$
\bar{\lambda}_{2}^{(1,1,0)}=\frac{1}{2} \bar{\lambda}_{1}^{(0,2)} .
$$

12 Eq. (A.1) evidently follows from (A.3) and (A.4).

\section{Appendix B Second-order expansion of the dimorphic fitness $\lambda_{2}\left(\varepsilon, \theta, \Delta x^{\prime}\right)$}

${ }_{14}$ In this appendix we make use of the consistency property $\mathrm{C} 1$, linking the dimorphic to the monomorphic fitness, and of properties P1-P4 (see Sect.2.1) to compute step by step the expansion (28) up to second 16 order.

We start by noting that the $x^{\prime}$-derivatives of $\mathrm{C} 1$ imply

$$
\bar{\lambda}_{2}^{(0,0, q)}=\bar{\lambda}_{1}^{(0, q)}, \quad q \geq 0,
$$

${ }_{18}$ and by recalling that over-bars evaluations are taken at $\left(\varepsilon, \Delta x^{\prime}\right)=(0,0)$ for $\lambda_{2}\left(\varepsilon, \theta, \Delta x^{\prime}\right)$ and at $(\bar{x}, \bar{x})$ for $\lambda_{1}\left(x, x^{\prime}\right)$. The zero-order term $\bar{\lambda}_{2}$ and the first-order term $\bar{\lambda}_{2}^{(0,0,1)}$ are therefore null by the neutrality and 20 singularity conditions (12) and (5), respectively.

More involved is the computation of the other first-order term, i.e., showing $\bar{\lambda}_{2}^{(1,0,0)}=0$ (see Tab.3). 22 Computing $\bar{\lambda}_{2}^{(1,0, q)}, q \geq 0$, from the $\lambda_{2}$ definition (26), we obtain

$$
\begin{aligned}
& \bar{\lambda}_{2}^{(1,0, q)}=\left[g^{(1,0,0,0, q)}\left(-\bar{r}^{(1,0)} s_{f}+(1-\bar{r})\left(s_{f}^{(1,0,0)} \bar{r}^{(1,0)}+s_{f}^{(0,1,0)}\right)\right)+\right. \\
& g^{(0,1,0,0, q)}\left(\bar{r}^{(1,0)} s_{f}+\bar{r}\left(s_{f}^{(1,0,0)} \bar{r}^{(1,0)}+s_{f}^{(0,1,0)}\right)\right)+ \\
&\left.g^{(0,0,1,0, q)} \cos \theta+g^{(0,0,0,1, q)} \sin \theta\right]\left.\right|_{\varepsilon=0, \Delta x^{\prime}=0},
\end{aligned}
$$

where functions' arguments, here omitted, are as in (26). The right-hand side of (B.2) simplifies by taking 24 into account that

$$
\left.g^{(1,0,0,0, q)}\right|_{\varepsilon=0, \Delta x^{\prime}=0}=\left.g^{(0,1,0,0, q)}\right|_{\varepsilon=0, \Delta x^{\prime}=0}=\bar{g}^{(1,0,0,0, q)}
$$

by $\mathrm{P} 2$ and that

$$
\begin{aligned}
\left.g^{(0,0,1,0, q)}\right|_{\varepsilon=0, \Delta x^{\prime}=0} & =\bar{\phi}_{1,1}^{(0,0, q)}(1-\bar{r}(0, \theta)) s_{f}(\bar{r}(0, \theta), 0, \theta), \\
\left.g^{(0,0,0,1, q)}\right|_{\varepsilon=0, \Delta x^{\prime}=0} & =\bar{\phi}_{1,1}^{(0,0, q)} \bar{r}(0, \theta) s_{f}(\bar{r}(0, \theta), 0, \theta)
\end{aligned}
$$


by $\mathrm{P} 4$ and $\mathrm{P} 3,4 \mathrm{a}$, respectively. The result is

$$
\begin{aligned}
\bar{\lambda}_{2}^{(1,0, q)}=\bar{g}^{(1,0,0,0, q)}\left(s_{f}^{(1,0,0)}(\bar{r}(0, \theta), 0, \theta) \bar{r}^{(1,0)}(0, \theta)+s_{f}^{(0,1,0)}(\bar{r}(0, \theta), 0, \theta)\right)+ \\
\bar{\phi}_{1,1}^{(0,0, q)} s_{f}(\bar{r}(0, \theta), 0, \theta)((1-\bar{r}(0, \theta)) \cos \theta+\bar{r}(0, \theta) \sin \theta) .
\end{aligned}
$$

Substituting in (B.5) the expressions in Tab. 1 for the fast-equilibrium manifold $s_{f}(\bar{r}(0, \theta), 0, \theta)$ and for the derivatives $s_{f}^{(1,0,0)}(\bar{r}(0, \theta), 0, \theta)$ and $s_{f}^{(0,1,0)}(\bar{r}(0, \theta), 0, \theta)$ (computed below in Sect. B.1), we obtain

$$
\bar{\lambda}_{2}^{(1,0, q)}=\left(\bar{g}^{(1,0,0,0, q)} \bar{n}^{(1)}+\bar{\phi}_{1,1}^{(0,0, q)} \bar{n}\right)((1-\bar{r}(0, \theta)) \cos \theta+\bar{r}(0, \theta) \sin \theta),
$$

that further simplifies to

$$
\bar{\lambda}_{2}^{(1,0, q)}=((1-\bar{r}(0, \theta)) \cos \theta+\bar{r}(0, \theta) \sin \theta) \bar{\lambda}_{1}^{(1, q)}
$$

4 by taking Eq. (11b) into account. With $q=0$, neutrality (13a) and singularity (5) yield $\bar{\lambda}_{2}^{(1,0,0)}=0$, whereas substituting the expression for $\bar{r}(0, \theta)$ from Tab. 2 (computed below in Sect.B.2) into (B.6) with $q=1$, we 6 obtain

$$
\bar{\lambda}_{2}^{(1,0,1)}=-\frac{1}{2}(\sin \theta+\cos \theta) \bar{\lambda}_{1}^{(0,2)},
$$

as in Tab. 3.

We finally need to compute $\bar{\lambda}_{2}^{(2,0,0)}$. Again from the $\lambda_{2}$ definition (26), we have

$$
\begin{aligned}
& \bar{\lambda}_{2}^{(2,0,0)}=\left[\left(g^{(2,0,0,0,0)}\left(-\bar{r}^{(1,0)} s_{f}+(1-\bar{r})\left(s_{f}^{(1,0,0)} \bar{r}^{(1,0)}+s_{f}^{(0,1,0)}\right)\right)+\right.\right. \\
& \left.g^{(1,1,0,0,0)}\left(\bar{r}^{(1,0)} s_{f}+\bar{r}\left(s_{f}^{(1,0,0)} \bar{r}^{(1,0)}+s_{f}^{(0,1,0)}\right)\right)+g^{(1,0,1,0,0)} \cos \theta+g^{(1,0,0,1,0)} \sin \theta\right) \times \\
& \left(-\bar{r}^{(1,0)} s_{f}+(1-\bar{r})\left(s_{f}^{(1,0,0)} \bar{r}^{(1,0)}+s_{f}^{(0,1,0)}\right)\right)+ \\
& g^{(1,0,0,0,0)}\left(-\bar{r}^{(2,0)} s_{f}-2 \bar{r}^{(1,0)}\left(s_{f}^{(1,0,0)} \bar{r}^{(1,0)}+s_{f}^{(0,1,0)}\right)+\right. \\
& \left.(1-\bar{r})\left(\left(s_{f}^{(2,0,0)} \bar{r}^{(1,0)}+2 s_{f}^{(1,1,0)}\right) \bar{r}^{(1,0)}+s_{f}^{(1,0,0)} \bar{r}^{(2,0)}+s_{f}^{(0,2,0)}\right)\right)+ \\
& \left(g^{(1,1,0,0,0)}\left(-\bar{r}^{(1,0)} s_{f}+(1-\bar{r})\left(s_{f}^{(1,0,0)} \bar{r}^{(1,0)}+s_{f}^{(0,1,0)}\right)\right)+\right. \\
& \left.g^{(0,2,0,0,0)}\left(\bar{r}^{(1,0)} s_{f}+\bar{r}\left(s_{f}^{(1,0,0)} \bar{r}^{(1,0)}+s_{f}^{(0,1,0)}\right)\right)+g^{(0,1,1,0,0)} \cos \theta+g^{(0,1,0,1,0)} \sin \theta\right) \times \\
& \left(\bar{r}^{(1,0)} s_{f}+\bar{r}\left(s_{f}^{(1,0,0)} \bar{r}^{(1,0)}+s_{f}^{(0,1,0)}\right)\right)+ \\
& g^{(0,1,0,0,0)}\left(\bar{r}^{(2,0)} s_{f}+2 \bar{r}^{(1,0)}\left(s_{f}^{(1,0,0)} \bar{r}^{(1,0)}+s_{f}^{(0,1,0)}\right)+\right. \\
& \left.\bar{r}\left(\left(s_{f}^{(2,0,0)} \bar{r}^{(1,0)}+2 s_{f}^{(1,1,0)}\right) \bar{r}^{(1,0)}+s_{f}^{(1,0,0)} \bar{r}^{(2,0)}+s_{f}^{(0,2,0)}\right)\right)+ \\
& \cos \theta\left(g^{(1,0,1,0,0)}\left(-\bar{r}^{(1,0)} s_{f}+(1-\bar{r})\left(s_{f}^{(1,0,0)} \bar{r}^{(1,0)}+s_{f}^{(0,1,0)}\right)\right)+\right. \\
& \left.g^{(0,1,1,0,0)}\left(\bar{r}^{(1,0)} s_{f}+\bar{r}\left(s_{f}^{(1,0,0)} \bar{r}^{(1,0)}+s_{f}^{(0,1,0)}\right)\right)+g^{(0,0,2,0,0)} \cos \theta+g^{(0,0,1,1,0)} \sin \theta\right)+ \\
& \sin \theta\left(g^{(1,0,0,1,0)}\left(-\bar{r}^{(1,0)} s_{f}+(1-\bar{r})\left(s_{f}^{(1,0,0)} \bar{r}^{(1,0)}+s_{f}^{(0,1,0)}\right)\right)+\right. \\
& \left.\left.g^{(0,1,0,1,0)}\left(\bar{r}^{(1,0)} s_{f}+\bar{r}\left(s_{f}^{(1,0,0)} \bar{r}^{(1,0)}+s_{f}^{(0,1,0)}\right)\right)+g^{(0,0,1,1,0)} \cos \theta+g^{(0,0,0,2,0)} \sin \theta\right)\right]\left.\right|_{\varepsilon=0, \Delta x^{\prime}=0^{\circ}} .
\end{aligned}
$$

Applying P2, i.e., taking (B.3) and

$$
\left.g^{(2,0,0,0,0)}\right|_{\varepsilon=0, \Delta x^{\prime}=0}=\left.g^{(1,1,0,0,0)}\right|_{\varepsilon=0, \Delta x^{\prime}=0}=\left.g^{(0,2,0,0,0)}\right|_{\varepsilon=0, \Delta x^{\prime}=0}=\bar{g}^{(2,0,0,0,0)}
$$


into account, the right-hand side of (B.7) simplifies to

$$
\begin{aligned}
\bar{\lambda}_{2}^{(2,0,0)}=[ & \bar{g}^{(2,0,0,0,0)}\left(s_{f}^{(1,0,0)} \bar{r}^{(1,0)}+s_{f}^{(0,1,0)}\right)^{2}+ \\
& \bar{g}^{(1,0,0,0,0)}\left(\left(s_{f}^{(2,0,0)} \bar{r}^{(1,0)}+2 s_{f}^{(1,1,0)}\right) \bar{r}^{(1,0)}+s_{f}^{(1,0,0)} \bar{r}^{(2,0)}+s_{f}^{(0,2,0)}\right)+ \\
& 2 \cos \theta\left(g^{(1,0,1,0,0)}\left(-\bar{r}^{(1,0)} s_{f}+(1-\bar{r})\left(s_{f}^{(1,0,0)} \bar{r}^{(1,0)}+s_{f}^{(0,1,0)}\right)\right)+g^{(0,1,1,0,0)}\left(\bar{r}^{(1,0)} s_{f}+\bar{r}\left(s_{f}^{(1,0,0)} \bar{r}^{(1,0)}+s_{f}^{(0,1,0)}\right)\right)\right)+ \\
& 2 \sin \theta\left(g^{(0,1,0,1,0)}\left(\bar{r}^{(1,0)} s_{f}+\bar{r}\left(s_{f}^{(1,0,0)} \bar{r}^{(1,0)}+s_{f}^{(0,1,0)}\right)\right)+g^{(1,0,0,1,0)}\left(-\bar{r}^{(1,0)} s_{f}+(1-\bar{r})\left(s_{f}^{(1,0,0)} \bar{r}^{(1,0)}+s_{f}^{(0,1,0)}\right)\right)\right)+ \\
& \left.g^{(0,0,2,0,0)} \cos ^{2} \theta+2 g^{(0,0,1,1,0)} \sin \theta \cos \theta+g^{(0,0,0,2,0)} \sin ^{2} \theta\right]\left.\right|_{\varepsilon=0, \Delta x^{\prime}=0}
\end{aligned}
$$

2 Applying P4, P3,4a, and P1-4, i.e.,

$$
\begin{aligned}
\left.g^{(1,0,1,0,0)}\right|_{\varepsilon=0, \Delta x^{\prime}=0} & =\bar{\phi}_{1,1}+\bar{\phi}_{1,1}^{(1,0,0)}(1-\bar{r}(0, \theta)) s_{f}(\bar{r}(0, \theta), 0, \theta), \\
\left.g^{(0,1,1,0,0)}\right|_{\varepsilon=0, \Delta x^{\prime}=0} & =\bar{\phi}_{1,1}^{(1,0,0)}(1-\bar{r}(0, \theta)) s_{f}(\bar{r}(0, \theta), 0, \theta), \\
\left.g^{(0,1,0,1,0)}\right|_{\varepsilon=0, \Delta x^{\prime}=0} & =\bar{\phi}_{1,1}+\bar{\phi}_{1,1}^{(1,0,0)} \bar{r}(0, \theta) s_{f}(\bar{r}(0, \theta), 0, \theta), \\
\left.g^{(1,0,0,1,0)}\right|_{\varepsilon=0, \Delta x^{\prime}=0} & =\bar{\phi}_{1,1}^{(1,0,0)} \bar{r}(0, \theta) s_{f}(\bar{r}(0, \theta), 0, \theta), \\
\left.g^{(0,0,2,0,0)}\right|_{\varepsilon=0, \Delta x^{\prime}=0} & =\bar{\phi}_{2,1}(1-\bar{r}(0, \theta)) s_{f}(\bar{r}(0, \theta), 0, \theta)+\bar{\phi}_{2,2}(1-\bar{r}(0, \theta))^{2} s_{f}(\bar{r}(0, \theta), 0, \theta)^{2}, \\
\left.g^{(0,0,1,1,0)}\right|_{\varepsilon=0, \Delta x^{\prime}=0} & =\bar{\phi}_{2,2}(1-\bar{r}(0, \theta)) \bar{r}(0, \theta) s_{f}(\bar{r}(0, \theta), 0, \theta)^{2}, \\
\left.g^{(0,0,0,2,0)}\right|_{\varepsilon=0, \Delta x^{\prime}=0} & =\bar{\phi}_{2,1} \bar{r}(0, \theta) s_{f}(\bar{r}(0, \theta), 0, \theta)+\bar{\phi}_{2,2} \bar{r}(0, \theta)^{2} s_{f}(\bar{r}(0, \theta), 0, \theta)^{2},
\end{aligned}
$$

the right-hand side of (B.8) becomes

$$
\begin{aligned}
\bar{\lambda}_{2}^{(2,0,0)}= & \bar{g}^{(2,0,0,0,0)}\left(s_{f}^{(1,0,0)}(\bar{r}(0, \theta), 0, \theta) \bar{r}^{(1,0)}(0, \theta)+s_{f}^{(0,1,0)}(\bar{r}(0, \theta), 0, \theta)\right)^{2}+\bar{g}^{(1,0,0,0,0)} \times \\
& \left(\left(s_{f}^{(2,0,0)}(\bar{r}(0, \theta), 0, \theta) \bar{r}^{(1,0)}(0, \theta)+2 s_{f}^{(1,1,0)}(\bar{r}(0, \theta), 0, \theta)\right) \bar{r}^{(1,0)}(0, \theta)+s_{f}^{(1,0,0)}(\bar{r}(0, \theta), 0, \theta) \bar{r}^{(2,0)}(0, \theta)+s_{f}^{(0,2,0)}(\bar{r}(0, \theta), 0, \theta)\right)+ \\
& 2\left(\bar{\phi}_{1,1}+\bar{\phi}_{1,1}^{(1,0,0)} s_{f}(\bar{r}(0, \theta), 0, \theta)\right)((1-\bar{r}(0, \theta)) \cos \theta+\bar{r}(0, \theta) \sin \theta)\left(s_{f}^{(1,0,0)}(\bar{r}(0, \theta), 0, \theta) \bar{r}^{(1,0)}(0, \theta)+s_{f}^{(0,1,0)}(\bar{r}(0, \theta), 0, \theta)\right)+ \\
& 2 \bar{\phi}_{1,1} s_{f}(\bar{r}(0, \theta), 0, \theta) \bar{r}^{(1,0)}(0, \theta)(\sin \theta-\cos \theta)+ \\
& \bar{\phi}_{2,1} s_{f}(\bar{r}(0, \theta), 0, \theta)\left((1-\bar{r}(0, \theta)) \cos ^{2} \theta+\bar{r}(0, \theta) \sin ^{2} \theta\right)+\bar{\phi}_{2,2} s_{f}(\bar{r}(0, \theta), 0, \theta)^{2}((1-\bar{r}(0, \theta)) \cos \theta+\bar{r}(0, \theta) \sin \theta)^{2} .
\end{aligned}
$$

4 Substituting in (B.10) the fast-equilibrium manifold $s_{f}(\bar{r}(0, \theta), 0, \theta)$ and its derivatives from Tab.1, we obtain

$$
\begin{aligned}
\bar{\lambda}_{2}^{(2,0,0)}= & \left(\bar{g}^{(2,0,0,0,0)}\left(\bar{n}^{(1)}\right)^{2}+2\left(\bar{\phi}_{1,1}+\bar{\phi}_{1,1}^{(1,0,0)} \bar{n}\right) \bar{n}^{(1)}+\bar{g}^{(1,0,0,0,0)} \bar{n}^{(2)}+\bar{\phi}_{2,1} \bar{n}+\bar{\phi}_{2,2} \bar{n}^{2}\right) \times \\
& ((1-\bar{r}(0, \theta)) \cos \theta+\bar{r}(0, \theta) \sin \theta)^{2} \\
+ & 2 \bar{r}^{(1,0)}(0, \theta)(\sin \theta-\cos \theta)\left(\bar{g}^{(1,0,0,0,0)} \bar{n}^{(1)}+\bar{\phi}_{1,1} \bar{n}\right)-\bar{r}(0, \theta)(1-\bar{r}(0, \theta))(\sin \theta-\cos \theta)^{2} \bar{\lambda}_{1}^{(0,2)},(B .
\end{aligned}
$$

6 that further simplifies taking (11c) $(q=0)$ into account and noting that, from the definition $(2)$ of the resident equilibrium and $\mathrm{P} 1,4$, we have

$$
\bar{n}^{(1)}=-\frac{\bar{\phi}_{1,1} \bar{n}+\bar{g}^{(0,0,0,0,1)}}{\bar{g}^{(1,0,0,0,0)}} \stackrel{(5,11 a)}{=}-\frac{\bar{\phi}_{1,1} \bar{n}}{\bar{g}^{(1,0,0,0,0)}} .
$$

8 Thanks to (B.12) we lose the $\bar{r}^{(1,0)}(0, \theta)$-term in (B.11) and obtain

$$
\begin{aligned}
\bar{\lambda}_{2}^{(2,0,0)=} & ((1-\bar{r}(0, \theta)) \cos \theta+\bar{r}(0, \theta) \sin \theta)^{2} \bar{\lambda}_{1}^{(2,0)}-\bar{r}(0, \theta)(1-\bar{r}(0, \theta))(\cos \theta-\sin \theta)^{2} \bar{\lambda}_{1}^{(0,2)} \\
\stackrel{(13 \mathrm{~b})}{=} & -2((1-\bar{r}(0, \theta)) \cos \theta+\bar{r}(0, \theta) \sin \theta)^{2} \bar{\lambda}_{1}^{(1,1)} \\
& -\left(((1-\bar{r}(0, \theta)) \cos \theta+\bar{r}(0, \theta) \sin \theta)^{2}+\bar{r}(0, \theta)(1-\bar{r}(0, \theta))(\sin \theta-\cos \theta)^{2}\right) \bar{\lambda}_{1}^{(0,2)} .
\end{aligned}
$$

Substituting the expression for $\bar{r}(0, \theta)$ from Tab.2, we finally obtain the expression in Tab.3, i.e.,

$$
\bar{\lambda}_{2}^{(2,0,0)}=\sin \theta \cos \theta \bar{\lambda}_{1}^{(0,2)} .
$$




\section{Appendix B.1. Derivatives of the fast-equilibrium manifold $s_{f}(r, \varepsilon, \theta)$}

2 The derivatives w.r.t. $(r, \varepsilon)$ of the fast-equilibrium manifold are obtained by differentiating the definition (23) and by applying property P1-P4. For notation convenience, we omit functions' arguments and denote 4 by $g(1)$ and $g(2)$ the evaluations

$$
g\left((1-r) s_{f}(r, \varepsilon, \theta), r s_{f}(r, \varepsilon, \theta), \bar{x}+\varepsilon \cos \theta, \bar{x}+\varepsilon \sin \theta, \bar{x}+\varepsilon \cos \theta\right)
$$

and

$$
g\left((1-r) s_{f}(r, \varepsilon, \theta), r s_{f}(r, \varepsilon, \theta), \bar{x}+\varepsilon \cos \theta, \bar{x}+\varepsilon \sin \theta, \bar{x}+\varepsilon \sin \theta\right)
$$

6 appearing in the definition (23) (and differing only in the last argument equal to $x_{1}$ and $x_{2}$, respectively).

First note that the evaluation at $\varepsilon=0$ of Eq. (23) and P2 give

$$
g\left(s_{f}(r, 0, \theta), 0, \bar{x}, \bar{x}, \bar{x}\right)=0,
$$

8 that compared with the definition (2) of the resident equilibrium gives the zero-order term $s_{f}(r, 0, \theta)=\bar{n}(\bar{x})$ in Tab.1.

The $r$-derivative of Eq. (23), i.e.,

$$
\begin{aligned}
& {\left[(1-r)\left(g^{(1,0,0,0,0)}(1)\left(-s_{f}+(1-r) s_{f}^{(1,0,0)}\right)+g^{(0,1,0,0,0)}(1)\left(s_{f}+r s_{f}^{(1,0,0)}\right)\right)-g(1)+\right.} \\
& \left.\quad r\left(g^{(1,0,0,0,0)}(2)\left(-s_{f}+(1-r) s_{f}^{(1,0,0)}\right)+g^{(0,1,0,0,0)}(2)\left(s_{f}+r s_{f}^{(1,0,0)}\right)\right)+g(2)\right]\left.\right|_{\varepsilon=0}=0,
\end{aligned}
$$

simplifies to

$$
\bar{g}^{(1,0,0,0,0)} s_{f}^{(1,0,0)}(r, 0, \theta)=0
$$

using P2 (i.e., taking (B.3) into account) and noting that

$$
\left.g(1)\right|_{\varepsilon=0}=\left.g(2)\right|_{\varepsilon=0}=g\left(s_{f}(r, 0, \theta), 0, \bar{x}, \bar{x}, \bar{x}\right)=0
$$

by Eq. (B.13). Being $\bar{g}^{(1,0,0,0,0)}<0$ by the hyperbolic stability of the resident equilibrium $\bar{n}(\bar{x}$ ) (see Eq. ${ }_{14}(10)$ ), we conclude $s_{f}^{(1,0,0)}(r, 0, \theta)=0$ (as in Tab. 1 ).

The $\varepsilon$-derivative of Eq. (23), i.e.,

$$
\begin{gathered}
{\left[( 1 - r ) \left(g^{(1,0,0,0,0)}(1)(1-r) s_{f}^{(0,1,0)}+g^{(0,1,0,0,0)}(1) r s_{f}^{(0,1,0)}+\right.\right.} \\
\left.g^{(0,0,1,0,0)}(1) \cos \theta+g^{(0,0,0,1,0)}(1) \sin \theta+g^{(0,0,0,0,1)}(1) \cos \theta\right)+ \\
r\left(g^{(1,0,0,0,0)}(2)(1-r) s_{f}^{(0,1,0)}+g^{(0,1,0,0,0)}(2) r s_{f}^{(0,1,0)}+\right. \\
\left.\left.\quad g^{(0,0,1,0,0)}(2) \cos \theta+g^{(0,0,0,1,0)}(2) \sin \theta+g^{(0,0,0,0,1)}(2) \sin \theta\right)\right]\left.\right|_{\varepsilon=0}=0
\end{gathered}
$$

16 simplifies to

$$
\bar{g}^{(1,0,0,0,0)} s_{f}^{(0,1,0)}(r, 0, \theta)+\left(\bar{\phi}_{1,1} \bar{n}+\bar{g}^{(0,0,0,0,1)}\right)((1-r) \cos \theta+r \sin \theta)=0
$$

using P2 (i.e., taking (B.3) into account), P4, and P3,4. Then, using Eq. (B.12), we obtain

$$
s_{f}^{(0,1,0)}(r, 0, \theta)=((1-r) \cos \theta+r \sin \theta) \bar{n}^{(1)}
$$

${ }_{18}$ (as in Tab.1).

The computation of the derivatives $s_{f}^{(1,1,0)}(r, 0, \theta)$ and $s_{f}^{(0,2,0)}(r, 0, \theta)$ that appear in Eq. (B.10) is left in the Supplementary Material.

\section{Appendix B.2. Derivatives of the slow equilibrium $\bar{r}(\varepsilon, \theta)$}


The derivatives w.r.t. $\varepsilon$ of the slow equilibrium are obtained by $\varepsilon$-differentiating the definition (24) and by 2 applying property P1-P4. For notation convenience, we omit functions' arguments and denote by $g(1)$ and $g(2)$ the evaluations

$$
g\left((1-\bar{r}(\varepsilon, \theta)) s_{f}(\bar{r}(\varepsilon, \theta), \varepsilon, \theta), \bar{r}(\varepsilon, \theta) s_{f}(\bar{r}(\varepsilon, \theta), \varepsilon, \theta), \bar{x}+\varepsilon \cos \theta, \bar{x}+\varepsilon \sin \theta, \bar{x}+\varepsilon \cos \theta\right)
$$

4 and

$$
g\left((1-\bar{r}(\varepsilon, \theta)) s_{f}(\bar{r}(\varepsilon, \theta), \varepsilon, \theta), \bar{r}(\varepsilon, \theta) s_{f}(\bar{r}(\varepsilon, \theta), \varepsilon, \theta), \bar{x}+\varepsilon \cos \theta, \bar{x}+\varepsilon \sin \theta, \bar{x}+\varepsilon \sin \theta\right)
$$

appearing in the definition (24) (and differing only in the last argument equal to $x_{1}$ and $x_{2}$, respectively).

The $\varepsilon$-derivative of (24) simply gives

$$
\left.g^{(0,0,0,0,1)}(2)\right|_{\varepsilon=0} \sin \theta-\left.g^{(0,0,0,0,1)}(1)\right|_{\varepsilon=0} \cos \theta \stackrel{\mathrm{P} 2}{=} \bar{g}^{(0,0,0,0,1)}(\sin \theta-\cos \theta)=0,
$$

as all derivatives not involving the last argument cancel in the difference $g(2)-g(1)$. Eq. (B.14) is however $\therefore$ an identity, being $\bar{g}^{(0,0,0,0,1)}=\bar{\lambda}_{1}^{(0,1)}=0$ (see Eq. (11a) with $q=1$ and the singularity condition (5)).

We therefore need to take the second $\varepsilon$-derivative of (24), i.e.,

$$
\begin{aligned}
{[\sin \theta(} & 2 g^{(1,0,0,0,1)}(2)\left(-\bar{r}^{(1,0)} s_{f}+(1-\bar{r})\left(s_{f}^{(1,0,0)} \bar{r}^{(1,0)}+s_{f}^{(0,1,0)}\right)\right)+ \\
& 2 g^{(0,1,0,0,1)}(2)\left(\bar{r}^{(1,0)} s_{f}+\bar{r}\left(s_{f}^{(1,0,0)} \bar{r}^{(1,0)}+s_{f}^{(0,1,0)}\right)\right)+ \\
& \left.2 g^{(0,0,1,0,1)}(2) \cos \theta+2 g^{(0,0,0,1,1)}(2) \sin \theta+g^{(0,0,0,0,2)}(2) \sin \theta\right)+ \\
-\cos \theta( & 2 g^{(1,0,0,0,1)}(1)\left(-\bar{r}^{(1,0)} s_{f}+(1-\bar{r})\left(s_{f}^{(1,0,0)} \bar{r}^{(1,0)}+s_{f}^{(0,1,0)}\right)\right)+ \\
& 2 g^{(0,1,0,0,1)}(1)\left(\bar{r}^{(1,0)} s_{f}+\bar{r}\left(s_{f}^{(1,0,0)} \bar{r}^{(1,0)}+s_{f}^{(0,1,0)}\right)\right)+ \\
& \left.\left.2 g^{(0,0,1,0,1)}(1) \cos \theta+2 g^{(0,0,0,1,1)}(1) \sin \theta+g^{(0,0,0,0,2)}(1) \cos \theta\right)\right]\left.\right|_{\varepsilon=0}=0,
\end{aligned}
$$

that simplifies into

$$
\begin{aligned}
2 \bar{g}^{(1,0,0,0,1)} & \left(s_{f}^{(1,0,0)}(\bar{r}(0, \theta), 0, \theta) \bar{r}^{(1,0)}(0, \theta)+s_{f}^{(0,1,0)}(\bar{r}(0, \theta), 0, \theta)\right)+ \\
& 2 \bar{\phi}_{1,1}^{(0,0,1)} s_{f}(\bar{r}(0, \theta), 0, \theta)((1-\bar{r}(0, \theta)) \cos \theta+\bar{r}(0, \theta) \sin \theta)+\bar{g}^{(0,0,0,0,2)}(\sin \theta+\cos \theta)=0
\end{aligned}
$$

10 using P2, P4, and P3,4 (i.e., taking (B.3) and (B.4) into account) and removing the factor $(\sin \theta-\cos \theta)>$ 0 (recall Eq. (20b)). Substituting in (B.15) the expressions in Tab. 1 for the fast-equilibrium manifold $12 s_{f}(\bar{r}(0, \theta), 0, \theta)$ and for the derivatives $s_{f}^{(1,0,0)}(\bar{r}(0, \theta), 0, \theta)$ and $s_{f}^{(0,1,0)}(\bar{r}(0, \theta), 0, \theta)$ (computed in Sect. B.1), we obtain

$$
2\left(\bar{g}^{(1,0,0,0,1)} \bar{n}^{(1)}+\bar{\phi}_{1,1}^{(0,0,1)} \bar{n}\right)((1-\bar{r}(0, \theta)) \cos \theta+\bar{r}(0, \theta) \sin \theta)+\bar{g}^{(0,0,0,0,2)}(\sin \theta+\cos \theta)=0,
$$

from which, taking Eqs. (11a) $(q=2)$ and (11b) $(q=1)$ into account, we conclude

$$
\bar{r}(0, \theta)=-\frac{2 \cos \theta \bar{\lambda}_{1}^{(1,1)}+(\sin \theta+\cos \theta) \bar{\lambda}_{1}^{(0,2)}}{2(\sin \theta-\cos \theta) \bar{\lambda}_{1}^{(1,1)}}
$$

${ }_{14}$ (as in Tab. 2).

The computation of the first derivative $\bar{r}^{(1,0)}(0, \theta)$, needed for the third-order in the expansion (28), is 16 left in the Supplementary Material.

\section{Appendix C The case of polymorphic and/or multispecies coevolution}

We allow in this appendix the resident and mutant populations 1 and 2 to interact and coevolve with $P$ other populations of the same or different species, with densities packed in vector $N(t) \in \mathbb{R}^{P}$ and corresponding strategies (finitely different from $x_{1}$ and $x_{2}$ in the case of conspecifics) packed in vector $X$ (multiple, mutationally independent, traits per population are also allowed [Dercole \& Rinaldi, 2008]). 
The resident-mutant model (1) then becomes

$$
\begin{aligned}
\dot{n}_{1} & =n_{1} g\left(n_{1}, n_{2}, N, x_{1}, x_{2}, X, x_{1}\right), \\
\dot{n}_{2} & =n_{2} g\left(n_{1}, n_{2}, N, x_{1}, x_{2}, X, x_{2}\right), \\
\dot{N} & =F\left(n_{1}, n_{2}, N, x_{1}, x_{2}, X\right),
\end{aligned}
$$

2 where the function vector $F$ collects the population growth rates of the $P$ other populations (each component given by the density $N_{p}$ multiplied by the per-capita growth rate of population $p, p=1, \ldots, P$ ) ${ }_{4}$ and $g\left(n_{1}, n_{2}, N, x_{1}, x_{2}, X, x^{\prime}\right)$ is the new $g$-function. Properties P1-P4 easily extend to the new $g$ and also apply to vector $F$. E.g., property $\mathrm{P} 1$ defines the functions

$$
\begin{aligned}
g_{1}\left(n_{1}, N, x_{1}, X, x^{\prime}\right) & :=g\left(n_{1}, 0, N, x_{1}, x_{2}, X, x^{\prime}\right), \\
F_{1}\left(n_{1}, N, x_{1}, X\right) & :=F\left(n_{1}, 0, N, x_{1}, x_{2}, X\right),
\end{aligned}
$$

and $\mathrm{P} 4$ reads

$$
\begin{aligned}
g^{\left(0,0,0, d_{1}, 0,0,0\right)}\left(n_{1}, n_{2}, N, x, x, X, x^{\prime}\right) & =\sum_{i_{1}=1}^{d_{1}} \phi_{d_{1}, i_{1}}\left(n_{1}+n_{2}, N, x, X, x^{\prime}\right) n_{1}^{i_{1}}, \\
g^{\left(0,0,0, d_{1}, d_{2}, 0,0\right)}\left(n_{1}, n_{2}, N, x, x, X, x^{\prime}\right) & =\sum_{i_{1}=1}^{d_{1}} \sum_{i_{2}=1}^{d_{2}} \phi_{d_{1}, d_{2}, i_{1}, i_{2}}\left(n_{1}+n_{2}, N, x, X, x^{\prime}\right) n_{1}^{i_{1}} n_{2}^{i_{2}}, \\
F^{\left(0,0,0, d_{1}, 0,0\right)}\left(n_{1}, n_{2}, N, x, x, X\right) & =\sum_{i_{1}=1}^{d_{1}} \psi_{d_{1}, i_{1}}\left(n_{1}+n_{2}, N, x, X\right) n_{1}^{i_{1}}, \\
F^{\left(0,0,0, d_{1}, d_{2}, 0\right)}\left(n_{1}, n_{2}, N, x, x, X\right) & =\sum_{i_{1}=1}^{d_{1}} \sum_{i_{2}=1}^{d_{2}} \psi_{d_{1}, d_{2}, i_{1}, i_{2}}\left(n_{1}+n_{2}, N, x, X\right) n_{1}^{i_{1}} n_{2}^{i_{2}},
\end{aligned}
$$

for suitable new functions $\phi_{d_{1}, i_{1}}$ and $\phi_{d_{1}, d_{2}, i_{1}, i_{2}}$ and suitable function vectors $\psi_{d_{1}, i_{1}}$ and $\psi_{d_{1}, d_{2}, i_{1}, i_{2}}$ (with relations $\psi_{1,1,1,1}=\psi_{2,2}, \psi_{2,1,1,1}=\psi_{1,2,1,1}=\frac{1}{3} \psi_{3,2}, \psi_{2,1,2,1}=\psi_{1,2,1,2}=\psi_{3,3}$, analogous to P1-4), $d_{1}, d_{2} \geq 1$ [Dercole, 2014].

We assume that for all values of the strategies $x_{1}$ and $X$ that we consider, population 1 stationarily coexists with the other $P$ interacting populations at a strictly positive and (hyperbolically) stable equilib12 rium of model (C.1 a,c) with $n_{2}=0$. The resident equilibrium densities, denoted with functions $\bar{n}\left(x_{1}, X\right)$ and $\bar{N}\left(x_{1}, X\right)$, are implicitly defined by

$$
\begin{aligned}
g_{1}\left(\bar{n}\left(x_{1}, X\right), \bar{N}\left(x_{1}, X\right), x_{1}, X, x_{1}\right) & =0, \\
F_{1}\left(\bar{n}\left(x_{1}, X\right), \bar{N}\left(x_{1}, X\right), x_{1}, X\right) & =0 .
\end{aligned}
$$

${ }_{14}$ By the hyperbolic stability of the resident equilibrium, i.e., the negative real part of the eigenvalues of the Jacobian matrix

$$
J\left(x_{1}, X\right)=\left[\begin{array}{cc}
\bar{n}\left(x_{1}, X\right) g_{1}^{(1,0,0,0,0)}\left(\bar{n}\left(x_{1}, X\right), \bar{N}\left(x_{1}, X\right), x_{1}, X, x_{1}\right) & \bar{n}\left(x_{1}, X\right) g_{1}^{(0,1,0,0,0)}\left(\bar{n}\left(x_{1}, X\right), \bar{N}\left(x_{1}, X\right), x_{1}, X, x_{1}\right) \\
F_{1}^{(1,0,0,0)}\left(\bar{n}\left(x_{1}, X\right), \bar{N}\left(x_{1}, X\right), x_{1}, X\right) & F_{1}^{(0,1,0,0)}\left(\bar{n}\left(x_{1}, X\right), \bar{N}\left(x_{1}, X\right), x_{1}, X\right)
\end{array}\right],
$$

16 also population 2 is able to coexist with the other $P$ interacting populations at a strictly positive (and hyperbolically stable) equilibrium $\left(\bar{n}\left(x_{2}, X\right), \bar{N}\left(x_{2}, X\right)\right)$ of model (C.1b,c) with $n_{1}=0$ and $x_{1} \simeq x_{2}$.

18 Thus, the resident-mutant model (C.1) admits the two monomorphic equilibria $\left(\bar{n}\left(x_{1}, X\right), 0, \bar{N}\left(x_{1}, X\right)\right)$ and $\left(0, \bar{n}\left(x_{2}, X\right), \bar{N}\left(x_{2}, X\right)\right)$ for all $x_{1}, x_{2}$, and $X$ that we consider.

The invasion fitness for a mutant strategy $x^{\prime} \simeq x$ is given by

$$
\lambda_{1}\left(x, X, x^{\prime}\right):=g_{1}\left(\bar{n}(x, X), \bar{N}(x, X), x, X, x^{\prime}\right) .
$$

To characterize the joint evolution of strategies $(x, X)$, one has to write the invasion fitnesses for the mutants of each of the resident strategies in $X$, that are each based on the corresponding resident-mutant 
model [Dercole \& Rinaldi, 2008]. The result is an AD canonical equation of the form

$$
\begin{aligned}
\dot{x} & =\frac{1}{2} \mu(x) \sigma(x)^{2} \bar{n}(x, X) \lambda_{1}^{(0,0,1)}(x, X, x), \\
\dot{X} & =\cdots
\end{aligned}
$$

Here we do not explicitly consider the evolution of the strategies in $X$, but rather treat $X$ as a vector of constant parameters. We assume that $(\bar{x}, \bar{X})$ is a stable equilibrium of the canonical equation (C.3)

4 (a convergence-stable singular coalition of strategies, in the AD jargon), i.e., $\bar{\lambda}_{1}^{(0,0,1)}=0$ holds together with similar relations for the selection gradients associated to the strategies in $X$ (over-bars here denote 6 evaluations at the singular coalition). For any given $X$ sufficiently close to $\bar{X}$, we define the function $\bar{x}(X)$ as the singular value for the strategy $x$ at the given $X$, i.e.,

$$
\lambda_{1}^{(0,0,1)}(\bar{x}(X), X, \bar{x}(X))=0, \quad \bar{x}(\bar{X})=\bar{x},
$$

8 as if the strategies in $X$ were not subject to the mutation-selection process. Note that $\bar{x}(X)$ is uniquely defined by (C.4) locally to $X=\bar{X}$ (by the implicit function theorem) under

$$
\bar{\lambda}_{1}^{(1,0,1)}+\bar{\lambda}_{1}^{(0,0,2)} \neq 0,
$$

which is granted by the resident-mutant coexistence condition (G1), now rewritten as

$$
\bar{\lambda}_{1}^{(1,0,1)}<0
$$

${ }_{10}$ and by the proximity to the branching bifurcation at which $\bar{\lambda}_{1}^{(0,0,2)}=0$. The quantity in (C.5) is hence negative and excludes that a collision between two solutions for $\bar{x}(X)$ occurs for $X$ close to $\bar{X}$ (recall

12 the comment below Eq. (14), though (C.5) is not here related to the assumed convergence stability of the singular coalition).

Thanks to the parametric definition of the singular strategy $\bar{x}(X)$ in (C.4), our analysis in Sects. 2.2 and 2.3 goes through, with the complicacy of the $P$ extra non-evolving coexisting populations (see the Supplementary Material, where all computations are performed taking the $P$ extra populations into account). Specifically, under (C.G1), a locally conical resident-mutant coexistence region is rooted at $(\bar{x}(X), \bar{x}(X))$

18 in the strategy plane $\left(x_{1}, x_{2}\right)$ and, for each point of the region, the coexistence equilibrium densities $\bar{n}_{1}\left(x_{1}, x_{2}, X\right), \bar{n}_{2}\left(x_{1}, x_{2}, X\right)$, and $\bar{N}\left(x_{1}, x_{2}, X\right)$ are positive and defined by

$$
\begin{array}{r}
g\left(\bar{n}_{1}\left(x_{1}, x_{2}, X\right), \bar{n}_{2}\left(x_{1}, x_{2}, X\right), \bar{N}\left(x_{1}, x_{2}, X\right), x_{1}, x_{2}, X, x_{1}\right)=0, \\
g\left(\bar{n}_{1}\left(x_{1}, x_{2}, X\right), \bar{n}_{2}\left(x_{1}, x_{2}, X\right), \bar{N}\left(x_{1}, x_{2}, X\right), x_{1}, x_{2}, X, x_{2}\right)=0, \\
F\left(\bar{n}_{1}\left(x_{1}, x_{2}, X\right), \bar{n}_{2}\left(x_{1}, x_{2}, X\right), \bar{N}\left(x_{1}, x_{2}, X\right), x_{1}, x_{2}, X\right)=0 .
\end{array}
$$

The coexistence region boundaries can be approximated as in Sect.2.2, where now

$$
x_{1}:=\bar{x}(X)+\varepsilon \cos \theta \text { and } x_{2}:=\bar{x}(X)+\varepsilon \sin \theta .
$$

The tangent direction and the curvature of the boundaries at $(\bar{x}(X), \bar{x}(X))$ are given by formulas that are 22 formally analogous to those in Eqs. (18), (19), (21), and (22), with the difference that the derivatives of $\lambda_{1}\left(x, X, x^{\prime}\right)$ are now evaluated at $(\bar{x}(X), X, \bar{x}(X))$ and not at $(\bar{x}, \bar{X}, \bar{x})$. For this, we use over-hats (evaluation 24 at the singular strategy $\bar{x}(X))$ instead of over-bars (evaluation at the singular coalition $(\bar{x}, \bar{X})$ ):

$$
\tan \theta_{\mathrm{T} 2}(0)=\frac{1}{\tan \theta_{\mathrm{T} 1}(0)}=-\frac{2 \hat{\lambda}_{1}^{(1,0,1)}+\hat{\lambda}_{1}^{(0,0,2)}}{\hat{\lambda}_{1}^{(0,0,2)}} \stackrel{(13 \mathrm{~b})}{=} \frac{\hat{\lambda}_{1}^{(2,0,0)}}{\hat{\lambda}_{1}^{(0,0,2)}}
$$

and

$$
\begin{aligned}
& \theta_{\mathrm{T} 2}^{(1)}(0)=\theta_{\mathrm{T} 1}^{(1)}(0)= \\
& \quad-\frac{4\left(\hat{\lambda}_{1}^{(1,0,1)}\right)^{2} \hat{\lambda}_{1}^{(0,0,3)}-2 \hat{\lambda}_{1}^{(1,0,1)} \hat{\lambda}_{1}^{(0,0,2)}\left(3 \hat{\lambda}_{1}^{(1,0,2)}-\hat{\lambda}_{1}^{(0,0,3)}\right)+\left(\hat{\lambda}_{1}^{(0,0,2)}\right)^{2}\left(3 \hat{\lambda}_{1}^{(2,0,1)}+\hat{\lambda}_{1}^{(0,0,3)}\right)}{6 \sqrt{2}\left(2\left(\hat{\lambda}_{1}^{(1,0,1)}\right)^{2}+2 \hat{\lambda}_{1}^{(1,0,1)} \hat{\lambda}_{1}^{(0,0,2)}+\left(\hat{\lambda}_{1}^{(0,0,2)}\right)^{2}\right)^{3 / 2}}
\end{aligned}
$$


(recall that $\theta_{\mathrm{T} i}(\varepsilon)=\theta_{\mathrm{T} i}(0)+\theta_{\mathrm{T} i}^{(1)}(0) \varepsilon$ approximates for small $|\varepsilon|$ the boundary $i$ on which $\bar{n}_{i}\left(x_{1}, x_{2}, X\right)=0$, $2 \quad i=1,2)$.

For $\left(x_{1}, x_{2}\right)$ in the resident-mutant coexistence region and $X$ sufficiently close to $\bar{X}$, the dimorphic 4 fitness reads:

$$
\lambda_{2}\left(x_{1}, x_{2}, X, x^{\prime}\right):=g\left(\bar{n}_{1}\left(x_{1}, x_{2}, X\right), \bar{n}_{2}\left(x_{1}, x_{2}, X\right), \bar{N}\left(x_{1}, x_{2}, X\right), x_{1}, x_{2}, X, x^{\prime}\right)
$$

(we keep using "monomorphic" and "dimorphic" to denote the situations before and after resident-mutant coexistence, though evolution could be polymorphic due to the presence of other conspecifics). Analogously to what done in Sect.2.3, the dimorphic fitness can be rewritten in terms of $\left(\varepsilon, \theta, X, \Delta x^{\prime}\right)$, with $\Delta x^{\prime}:=$ $x^{\prime}-\bar{x}(X)$, as

$$
\begin{aligned}
& \lambda_{2}\left(\varepsilon, \theta, X, \Delta x^{\prime}\right):= \\
& g\left((1-\bar{r}(\varepsilon, \theta, X)) s_{f}(\bar{r}(\varepsilon, \theta, X), \varepsilon, \theta, X), \bar{r}(\varepsilon, \theta, X) s_{f}(\bar{r}(\varepsilon, \theta, X), \varepsilon, \theta, X), N_{f}(\bar{r}(\varepsilon, \theta, X), \varepsilon, \theta, X), \bar{x}(X)+\varepsilon \cos \theta, \bar{x}(X)+\varepsilon \sin \theta, X, \bar{x}(X)+\Delta x^{\prime}\right),
\end{aligned}
$$

where $\left\{s_{f}(r, \varepsilon, \theta, X), N_{f}(r, \varepsilon, \theta, X), r \in[0,1]\right\}$ is the fast-equilibrium manifold of model (C.1), to which ${ }_{6} s:=n_{1}+n_{2}$ and $N$ converge at constant $r$, and $\bar{r}(\varepsilon, \theta, X)$ is the equilibrium of the slow variable $r$. The fast-equilibrium manifold and the slow equilibrium are respectively defined by

$$
\begin{aligned}
0 & =\dot{n}_{1}+\dot{n}_{2} \\
& =(1-r) g\left((1-r) s_{f}(r, \varepsilon, \theta, X), r s_{f}(r, \varepsilon, \theta, X), N_{f}(r, \varepsilon, \theta, X), \bar{x}+\varepsilon \cos \theta, \bar{x}+\varepsilon \sin \theta, X, \bar{x}+\varepsilon \cos \theta\right) \\
& +r g\left((1-r) s_{f}(r, \varepsilon, \theta, X), r s_{f}(r, \varepsilon, \theta, X), N_{f}(r, \varepsilon, \theta, X), \bar{x}+\varepsilon \cos \theta, \bar{x}+\varepsilon \sin \theta, X, \bar{x}+\varepsilon \sin \theta\right), \\
0 & =\dot{N}=F\left((1-r) s_{f}(r, \varepsilon, \theta, X), r s_{f}(r, \varepsilon, \theta, X), N_{f}(r, \varepsilon, \theta, X), \bar{x}+\varepsilon \cos \theta, \bar{x}+\varepsilon \sin \theta, X\right)
\end{aligned}
$$

8 and

$0=g\left((1-\bar{r}(\varepsilon, \theta, X)) s_{f}(\bar{r}(\varepsilon, \theta, X), \varepsilon, \theta, X), \bar{r}(\varepsilon, \theta, X) s_{f}(\bar{r}(\varepsilon, \theta, X), \varepsilon, \theta, X), N_{f}(\bar{r}(\varepsilon, \theta, X), \varepsilon, \theta, X), \bar{x}(X)+\varepsilon \cos \theta, \bar{x}(X)+\varepsilon \sin \theta, X, \bar{x}(X)+\varepsilon \sin \theta\right)$

$-g\left((1-\bar{r}(\varepsilon, \theta, X)) s_{f}(\bar{r}(\varepsilon, \theta, X), \varepsilon, \theta, X), \bar{r}(\varepsilon, \theta, X) s_{f}(\bar{r}(\varepsilon, \theta, X), \varepsilon, \theta, X), N_{f}(\bar{r}(\varepsilon, \theta, X), \varepsilon, \theta, X), \bar{x}(X)+\varepsilon \cos \theta, \bar{x}(X)+\varepsilon \sin \theta, X, \bar{x}(X)+\varepsilon \cos \theta\right)$

and their relevant derivatives are computed in the Supplementary Material.

The right-hand side in (C.7) can be Taylor expanded around $\left(\varepsilon, \Delta x^{\prime}\right)=(0,0)$ at given $(\theta, X)$. The result is fully analogous to that of Sect.2.3, so back in the variables $\Delta x_{1}:=x_{1}-\bar{x}(X)=\varepsilon \cos \theta$ and ${ }_{12} \Delta x_{2}:=x_{2}-\bar{x}(X)=\varepsilon \sin \theta$ (see the definitions in (C.6)) we can write

$$
\lambda_{2}\left(\varepsilon, \theta, X, \Delta x^{\prime}\right)=\tilde{\lambda}_{2}\left(\Delta x_{1}, \Delta x_{2}, X, \Delta x^{\prime}\right)+O\left(\left\|\left(\varepsilon, \Delta x^{\prime}\right)\right\|^{4}\right),
$$

with

$$
\begin{aligned}
& \tilde{\lambda}_{2}\left(\Delta x_{1}, \Delta x_{2}, X, \Delta x^{\prime}\right):= \\
& \left(\frac{1}{2} \hat{\lambda}_{1}^{(0,0,2)}-\frac{1}{4} \frac{\hat{\lambda}_{1}^{(0,0,2)} \hat{\lambda}_{1}^{(1,0,2)}}{\hat{\lambda}_{1}^{(1,0,1)}}\left(\Delta x_{1}+\Delta x_{2}\right)+\frac{1}{6} \hat{\lambda}_{1}^{(0,0,3)}\left(\Delta x_{1}+\Delta x_{2}+\Delta x^{\prime}\right)\right)\left(\Delta x^{\prime}-\Delta x_{1}\right)\left(\Delta x^{\prime}-\Delta x_{2}\right),
\end{aligned}
$$

that is formally identical to the right-hand side in (29) except for replacing the over-bar evaluations with 14 over-hat ones. Note however the new definitions of variables $\Delta x_{1}$ and $\Delta x_{2}$. They respectively measure the horizontal and vertical deviations of $x_{1}$ and $x_{2}$ from the singular point $(\bar{x}(X), \bar{x}(X))$ in the strategy plane $16\left(x_{1}, x_{2}\right)$. A change in $X$ (that does not explicitly appear in the right-hand side of (C.10) because hidden in the over-hat evaluations) at constant $\left(\Delta x_{1}, \Delta x_{2}\right)$ hence implies a change in $\left(x_{1}, x_{2}\right)$.

Thanks again the parametric definition of the singular strategy $\bar{x}(X)$ in (C.4), also the two canonical models presented in Sect. 3 (as well as their unfolding as $\bar{\lambda}_{1}^{(0,0,2)}$ moves across zero in Sect. 3 ) remain formally valid under (C.G1) and

$$
\bar{\lambda}_{1}^{(0,0,3)} \neq 0,
$$

${ }_{18}$ because $X$ sufficiently close to $\bar{X}$ guarantees $\hat{\lambda}_{1}^{(1,0,1)} \neq 0$ and $\hat{\lambda}_{1}^{(0,0,3)} \neq 0$, so we can simply replace overbars with over-hats in the canonical models. Note, in particular, that $\left(\Delta x_{1}, \Delta x_{2}\right)=(0,0)$ now stands for 
$\left(x_{1}, x_{2}\right)=(\bar{x}(X), \bar{x}(X))$ in Figs. 3 and 4, and that the central panel occurs when $\hat{\lambda}_{1}^{(0,0,2)}=0$, while the 2 discriminant between top and bottom panels is $\hat{\lambda}_{1}^{(0,0,3)} \gtrless 0$.

We are now ready to take into account that (some or all of) the strategies in $X$ are coevolving with $4 x$ according to the canonical equation (C.3) in the monomorphic phase, and with $x_{1}$ and $x_{2}$ according to the canonical model

$$
\begin{aligned}
& \dot{x}_{1}=\left(\hat{\lambda}_{1}^{(1,0,1)} \Delta x_{2}+\frac{1}{2} \hat{\lambda}_{1}^{(0,0,2)}\left(\Delta x_{1}+\Delta x_{2}\right)\right)\left(\frac{1}{2} \hat{\lambda}_{1}^{(0,0,2)}-\frac{1}{4} \frac{\hat{\lambda}_{1}^{(0,0,2)} \hat{\lambda}_{1}^{(1,0,2)}}{\hat{\lambda}_{1}^{(1,0,1)}}\left(\Delta x_{1}+\Delta x_{2}\right)+\frac{1}{6} \hat{\lambda}_{1}^{(0,0,3)}\left(2 \Delta x_{1}+\Delta x_{2}\right)\right) \\
& \dot{x}_{2}=\left(\hat{\lambda}_{1}^{(1,0,1)} \Delta x_{1}+\frac{1}{2} \hat{\lambda}_{1}^{(0,0,2)}\left(\Delta x_{1}+\Delta x_{2}\right)\right)\left(\frac{1}{2} \hat{\lambda}_{1}^{(0,0,2)}-\frac{1}{4} \frac{\hat{\lambda}_{1}^{(0,0,2)} \hat{\lambda}_{1}^{(1,0,2)}}{\hat{\lambda}_{1}^{(1,0,1)}}\left(\Delta x_{1}+\Delta x_{2}\right)+\frac{1}{6} \hat{\lambda}_{1}^{(0,0,3)}\left(\Delta x_{1}+2 \Delta x_{2}\right)\right) \\
& \dot{X}=\cdots
\end{aligned}
$$

6 (see Eqs. (34), (35), (37)) in the dimorphic phase. We assume that branching at the singular coalition $(\bar{x}, \bar{X})$ is possible only in strategy $x$. Branching in the strategies in $X$ can be similarly discussed, one by 8 one, simply focusing on each component of $X$ as "the small $x$." When branching is possible with respect to multiple strategies, only the fastest diverging branching (the one with largest $\bar{\lambda}_{1}^{(0,0,2)}$ ) generically develops, 10 the others being "missed" [Kisdi, 1999] (see below and also [Landi et al., 2013]).

If $\bar{\lambda}_{1}^{(0,0,2)}<0$ and $X$ is initially sufficiently close to $\bar{X}$, the situation is that of the left panels in Figs. 3 2 and 4 , so the singular coalition is a terminal point w.r.t. strategy $x$. Note that if $X$ is not sufficiently close to $\bar{X}$, it might be $\hat{\lambda}_{1}^{(0,0,2)}>0$, so the right panels apply. An incipient branching is therefore possible in strategy $x$ close to $\bar{x}(X)$, but the evolution of $X$ is much faster ( $X$ being far from equilibrium) and turns the situation to the left panels before branching could actually develop (thanks to the assumed convergence stability of the singular coalition).

A similar scenario of missed branching [Kisdi, 1999, Landi et al., 2013] occurs when point $\left(x_{1}(t), x_{2}(t)\right)$, moving in the strategy plane in accordance with the canonical equation (C.11) along an incipient branching, hits the boundaries of the resident-mutant coexistence region, which are moving themselves along with the strategies in $X$. However, this is (generically) not possible if $\bar{\lambda}_{1}^{(0,0,2)}>0$ with $X(0)$ sufficiently close to $\bar{X}$. In fact, look at the right panels in Figs. 3 and 4 and consider an initial condition for the incipient branching above the diagonal and on the anti-diagonal $\Delta x_{1}+\Delta x_{2}=0$ (i.e., $\Delta x_{1}(0)=-\varepsilon, \Delta x_{2}(0)=\varepsilon$, $\varepsilon>0$ small). From Eqs.(C.11a,b) we then see that $\dot{x}_{1}<0$ and $\dot{x}_{2}>0$ have opposite leading terms ${ }_{24}\left(-\dot{x}_{1}=\dot{x}_{2}=-\frac{1}{2} \hat{\lambda}_{1}^{(1,0,1)} \hat{\lambda}_{1}^{(0,0,2)} \varepsilon+O\left(\varepsilon^{2}\right)>0\right)$, so the diversification initially points in the direction of the anti-diagonal. Moreover, the branching population remains initially split into two halves, as we see from 26 the leading terms

$$
\tilde{n}_{1}\left(\Delta x_{1}, \Delta x_{2}\right)=\tilde{n}_{2}\left(\Delta x_{1}, \Delta x_{2}\right)=-\frac{1}{2} \hat{\lambda}_{1}^{(1,0,1)}+O(\varepsilon)
$$

in the scaled approximations obtained from Eqs. $(36,37)$ along the anti-diagonal. As a consequence, the strategies in $X$ initially remain under (nearly) neutral selection, the corresponding populations facing half of the $x$-residents with a trait increased by $\varepsilon$, the other half with the same trait decreased by $\varepsilon$. Since so the strategies in $X$ were close to equilibrium $((x, X) \simeq(\bar{x}, \bar{X}))$ before the splitting, the same condition is maintained during the initial phase of branching (i.e., $X$ stays close to the null-surface $\dot{X}=0$ ). The result 32 is that the evolution of $X$ according to the canonical equation (C.11) is initially slower than the divergence of $x_{1}$ from $x_{2}$, as typically observed in the numerical simulations (see e.g. Fig.7(a)), and slow is also the 34 corresponding movement of the coexistence region boundaries in the plane $\left(x_{1}, x_{2}\right)$. This prevents missing the branching.

If the initial condition for $\left(x_{1}, x_{2}\right)$ is not taken on the anti-diagonal (Fig. 7(b)) and/or that for $X$ is not taken at $\bar{X}$ (Fig. $7(\mathrm{c})$ ), the convergence stability of the singular coalition $(\bar{x}, \bar{X})$ and the initial evolution of $\left(x_{1}, x_{2}\right)$ toward the anti-diagonal (see the right panels in Figs. 3 and 4 and recall that the diagonal 
and the anti-diagonal are the stable and unstable eigenvectors of the equilibrium $(\bar{x}, \bar{x})$ in the normal 2 form $(34,35,37))$ make branching in strategy $x$ possible from many initial conditions close to $(\bar{x}, \bar{X})$.

We close this appendix by noting that a more rigorous (center-manifold) approach seems feasible to show that model $(34,35,37)$ (with $\bar{\lambda}_{1}^{(d, q)}$ replaced by over-bars evaluations $\bar{\lambda}_{1}^{(d, 0, q)}$ at the singular coalition $(\bar{x}, \bar{X})$ ) is a normal form also in the presence of other coevolving populations. That is, despite the nonsmoothness of the coexistence equilibrium densities $\bar{n}_{1}\left(x_{1}, x_{2}, X\right)$ and $\bar{n}_{2}\left(x_{1}, x_{2}, X\right)$, and the consequent nonsmoothness of the dimorphic fitness $\lambda_{2}\left(x_{1}, x_{2}, X, x^{\prime}\right)$, model (C.11) is desingularized and reducible to a two-dimensional $\bar{\lambda}_{1}^{(0,0,2)}$-parameterized center manifold close to the branching bifurcation. This would require a straightforward expansion of our dimorphic fitness approximation (C.10) w.r.t. $X$ around $\bar{X}$, 10 taking the definition of the singular strategy $\bar{x}(X)$ in (C.4) into account. But it would also require the definition of an invasion fitness for each of the coevolving strategies in $X$. This is left for future work.

\section{${ }_{12}$ References}

Allgower, E. L. \& Georg, K. [1990] Numerical Continuation Methods: An Introduction (Springer-Verlag, Berlin).

Champagnat, N., Ferrière, R. \& Méléard, S. [2006] "Unifying evolutionary dynamics: From individual stochastic processes to macroscopic models," Theor Popul Biol 69, 297-321.

Christiansen, F. B. [1991] "On conditions for evolutionary stability for a continuously varying character," Am. Nat. 138, 37-50.

Dercole, F. [2014] "The ecology of asexual pairwise interactions: A generalized law of mass action." ArXiv:1411.0075 [q-bio.PE].

Dercole, F., Dieckmann, U., Obersteiner, M. \& Rinaldi, S. [2008] "Adaptive dynamics and technological change," Technovation 28, 335-348.

Dercole, F., Ferrière, R. \& Rinaldi, S. [2002] "Ecological bistability and evolutionary reversals under asymmetrical competition," Evolution 56, 1081-1090.

Dercole, F., Ferrière, R. \& Rinaldi, S. [2010a] "Chaotic Red Queen coevolution in three-species food chains," P. Roy. Soc. Lond. B Bio. 277, 2321-2330.

Dercole, F. \& Geritz, S. [2015] "Unfolding the resident-invader dynamics of similar strategies," J. Theor. Biol. (submitted).

Dercole, F., Gragnani, A., Ferrière, R. \& Rinaldi, S. [2006] "Coevolution of slow-fast populations: evolutionary sliding, evolutionary pseudo-equilibria and complex Red Queen dynamics," P. Roy. Soc. Lond. B Bio. 273, 983-990.

32 Dercole, F., Irisson, J. O. \& Rinaldi, S. [2003] "Bifurcation analysis of a prey-predator coevolution model," SIAM J. Appl. Math. 63, 1378-1391.

34 Dercole, F., Prieu, C. \& Rinaldi, S. [2010b] "Technological change and fisheries sustainability: The point of view of adaptive dynamics," Ecol. Modelling 221, 379-387.

36 Dercole, F. \& Rinaldi, S. [2008] Analysis of Evolutionary Processes: The Adaptive Dynamics Approach and Its Applications (Princeton University Press).

38 Dercole, F. \& Rinaldi, S. [2010] "Evolutionary dynamics can be chaotic: A first example," Internat. J. Bifur. Chaos 11, 3473-3485.

40 Dhooge, A., Govaerts, W. \& Kuznetsov, Yu. A. [2002] "MATCONT: A MATLAB package for numerical bifurcation analysis of ODEs," ACM T. Math. Software 29, 141-164.

42 Dieckmann, U. [1997] "Can adaptive dynamics invade?" Trends Ecol. Evol. 12, 128-131.

Dieckmann, U. \& Law, R. [1996] "The dynamical theory of coevolution: A derivation from stochastic ecological processes," J. Math. Biol. 34, 579-612.

Dieckmann, U., Marrow, U. \& Law, R. [1995] "Evolutionary cycling in predator-prey interactions: Population dynamics and the Red Queen," J. Theoret. Biol. 176, 91-102.

Doebeli, M. \& Dieckmann, U. [2000] "Evolutionary branching and sympatric speciation caused by different types of ecological interactions," Am. Nat. 156, 77-101.

Doebeli, M. \& Ispolatov, I. [2010] "Continuously stable strategies as evolutionary branching points," $J$. Theor. Biol. 266, 529-535. 
Doebeli, M. \& Ruxton, G. D. [1997] "Evolution of dispersal rates in metapopulation models: Branching and cyclic dynamics in phenotype space," Evolution 51, 1730-1741.

Durinx, M. [2008] "Life amidst singularities," Doctoral thesis, Institute of Biology, Leiden University, The Netherlands.

Durinx, M., Metz, J. A. J. \& Meszéna, G. [2008] "Adaptive dynamics for physiologically structured population models," J. Math. Biol. 56, 673-742.

Eshel, I. [1983] "Evolutionary and continuous stability," J. Theor. Biol. 103, 99-111.

\& Eshel, I., Motro, U. \& Sansone, E. [1997] "Continuous stability and evolutionary convergence," J. Theor. Biol. 185, 333-343.

Gavrilets, S. [2004] Fitness Landscapes and the Origin of Species (Princeton University Press, Princeton, NJ).

Geritz, S. A. H. [2005] "Resident-invader dynamics and the coexistence of similar strategies," J. Math. Biol. 50, 67-82.

Geritz, S. A. H., Kisdi, E., Meszéna, G. \& Metz, J. A. J. [1998] "Evolutionarily singular strategies and the adaptive growth and branching of the evolutionary tree," Evol. Ecol. 12, 35-57.

Geritz, S. A. H., Kisdi, E., van der Meijden, E. \& Metz, J. A. J. [1999] "Evolutionarily dynamics of seed size and seedling competitive ability," Theor. Popul. Biol. 55, 324-343.

Geritz, S. A. H., Metz, J. A. J., Kisdi, E. \& Meszéna, G. [1997] "The dynamics of adaptation and evolutionary branching," Phys. Rev. Lett. 78, 2024-2027.

Govaerts, W. [2000] Numerical Methods for Bifurcations of Dynamical Equilibria (SIAM, Philadelphia, PA).

22 Ito, H. C. \& Dieckmann, U. [2014] "Evolutionary branching under slow directional evolution," J. Theor. Biol. 360, 290-314.

24 Kisdi, E. [1999] "Evolutionary branching under asymmetric competition," J. Theoret. Biol. 197, 149-162.

Kuznetsov, Yu. A. [2004] Elements of Applied Bifurcation Theory, 3rd ed. (Springer Verlag).

26 Landi, P. \& Dercole, F. [2014] "The evolution of fashion traits: Pure social interactions promote diversity," Proceedings of the 8th European Nonlinear Dynamics Conference ENOC 2014 (Vienna).

28 Landi, P., Dercole, F. \& Rinaldi, S. [2013] "Branching scenarios in eco-evolutionary prey-predator models," SIAM J. Appl. Math. 73, 1634-1658.

so Levins, R. [1968] Evolution in Changing Environments (Princeton University Press, Princeton, NJ).

Maynard Smith, J. \& Price, J. [1973] "The logic of animal conflicts," Nature 246, 15-18.

32 Meijer, H. G. E., Dercole, F. \& Oldeman, B. E. [2009] "Numerical bifurcation analysis," Encyclopedia of Complexity and System Science, ed. Meyers, R. A., pp. 6329-6352.

34 Meszéna, G., Gyllenberg, M., Jacobs, F. \& Metz, J. A. J. [2005] "Link between population dynamics and dynamics of Darwinian evolution," Phys. Rev. Lett. 95, 078195.

${ }_{36}$ Metz, J., Nisbet, R. \& Geritz, S. [1992] "How should we define fitness for general ecological scenarios?" Trends Ecol. Evol. 7, 198-202.

зв Metz, J. A. J., Geritz, S. A. H., Meszéna, G., Jacobs, F. J. A. \& van Heerwaarden, J. S. [1996] "Adaptive dynamics: A geometrical study of the consequences of nearly faithful reproduction," Stochastic and Spatial Structures of Dynamical Systems, eds. van Strien, S. J. \& Verduyn Lunel, S. M. (Elsevier Science), pp. 183-231.

${ }_{42}$ Priklopil, T. [2012] "On invasion boundaries and the unprotected coexistence of two strategies," J. Math. Biol. 64, 1137-1156.

${ }_{44}$ Taylor, P. D. [1989] "Evolutionary stability in one-parameter models under weak selection," Theor. Popul. Biol. 36, 125-143.

${ }_{4}$ Vincent, T. \& Brown, J. [2005] Evolutionary Game Theory, Natural Selection, and Darwinian Dynamics (Cambridge University Press).

${ }_{48}$ Vukics, A., Asboth, J. \& Meszéna, G. [2003] "Speciation in multidimensional evolutionary space," Phys. Rev. E 68, 041903.

50 Ziman, J. (ed.) [2000] Technological Innovation as an Evolutionary Process (Cambridge University Press, Cambridge, UK). 